\title{
On the Planning and Design Problem of Fog Networks
}

\author{
by
}

\section{Faisal Haider}

A thesis submitted to the Faculty of Graduate and Postdoctoral Affairs in partial fulfillment of the requirements for the degree of

\section{Master of Applied Science}

in

Electrical and Computer Engineering

\author{
Carleton University \\ Ottawa, Ontario
}

(C) 2017

Faisal Haider 


\section{Abstract}

Fog computing is a paradigm of geographically distributed computing residing at the edge of the network. Fog computing is made up of fog nodes providing compute, storage and networking services to end users. In this thesis, we propose an exact model for the planning and design problem of fog networks. The model simultaneously

determines the optimal location, the capacity and the number of fog node(s) as well as the interconnection between the installed fog nodes and the cloud, while minimizing the delay in the network and the amount of traffic going to the cloud. To address this multiobjective problem, three multiobjective optimization methods (weighted sum, hierarchical and trade-off) are evaluated. The CPLEX solver was used to optimize the model for the three methods with different problem sizes and the results are analyzed. The results show that, as the input size increases, the delay and the traffic also increase in a linear form; whereas the solution time increases in non-polynomial time. The weighted sum method was able to achieve the best trade-off results for the delay and the traffic, whereas the hierarchical method was able to return minimum delay but with worse traffic going to the cloud. As the model considers realistic edge device traffic parameters and constraints, it can be helpful in deploying fog networks in the current cloud computing architecture. 


\section{Acknowledgments}

First and foremost, I am extremely grateful to my supervisor Prof. Marc StHilaire for believing in me when I contacted him from the other side of the world. This work would not have been possible without his invaluable inspiration, guidance and friendly availability, in spite of his busy schedule. It has been great honour and privilege to work under your supervision.

I would like to thank Dr. Christian Makaya for his guidance and wise suggestions throughout the whole period of my research. I am also grateful to my friend and mentor Dr. Muhammad Raisul Alam for his insightful suggestions and input on optimization and his support to make Canada a second home for me. I acknowledge with gratitude Decheng Zhang for being extremely supportive and sharing his knowledge from the beginning of this research.

I would like to extend my thanks to all my friends and colleauges of our lab for providing a relaxed and engaging research environment. Special thanks goes to Ammar, Jean-Daniel and Afsane. My heartfelt appreciation goes to my friend Shoeb Shaikh and Jamil Sayeed, who always stood by me with smile and good wishes.

Finally, I owe the greatest debt to my parents for their never-ending affection,

love and patience to become who I am today. It is my great pleasure to dedicate this thesis to Saif, Salma, Luthful and my parents. 


\section{Table of Contents}

$\begin{array}{lll}\text { Abstract } & \text { ii }\end{array}$

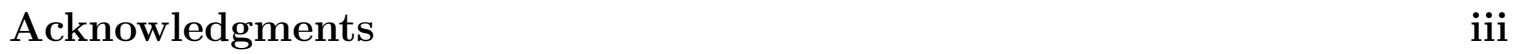

Table of Contents iv

List of Tables vii

$\begin{array}{ll}\text { List of Figures } & \text { ix }\end{array}$

List of Abbreviations xi

\begin{tabular}{lll}
\hline 1 & Introduction & 1
\end{tabular}

1.1 Problem Statement . . . . . . . . . . . . . . . . . . . . . . . 2

1.2 Research Objectives. . . . . . . . . . . . . . . . . . . . 3

1.3 Methodology . . . . . . . . . . . . . . . . . 4

1.4 Thesis Contributions $\ldots \ldots \ldots \ldots \ldots \ldots$

1.5 Thesis overview $\ldots \ldots \ldots \ldots \ldots \ldots \ldots \ldots \ldots \ldots$

\begin{tabular}{|lll}
\hline 2 & Background and Related Work & 7
\end{tabular}

2.1 Introduction to Cloud Computing . . . . . . . . . . . . . 7

$2.1 .1 \quad$ Definitions . . . . . . . . . . . . . . 8

2.1 .2 Related Technologies. . . . . . . . . . . . . . . . . . 9 9

2.1 .3 Service Models . . . . . . . . . . . . . . . . . . . . . . 11

2.1 .4 Deployment Models . . . . . . . . . . . . . . . . . . . . . 12

2.1 .5 VM Placement in Cloud . . . . . . . . . . . . . . . . . 13

2.1 .6 Limitations . . . . . . . . . . . . . . . . . . 15

2.2 Fog Computing $\ldots \ldots \ldots \ldots \ldots \ldots \ldots$ 
2.2 .1 Architecture . . . . . . . . . . . . . . . . 16

2.2 .2 Protocol $\ldots \ldots \ldots \ldots \ldots \ldots \ldots$

2.2 .3 Application . . . . . . . . . . . . . . . . . . . . . . . . . 19

2.2 .4 Current Research Related to Fog Computing . . . . . . . . . 21

2.3 Network Planning Process $\ldots \ldots \ldots$

2.4 Chapter Summary $\ldots \ldots \ldots \ldots \ldots$

\begin{tabular}{|ll|}
\hline 3 & Mathematical Model for Fog Planning Problem \\
\hline
\end{tabular}

3.1 Model Formulation . . . . . . . . . . . . . . . . . . . . . . . . . 27

3.1 .1 Assumptions . . . . . . . . . . . . . . . . . . . 27

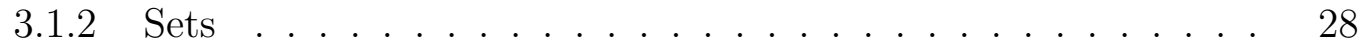

3.1 .3 Constants $\ldots \ldots \ldots \ldots \ldots$

3.1 .4 Functions . . . . . . . . . . . . . . . . . . . . . 29

3.1 .5 Decision Variables . . . . . . . . . . . . . . . . 30

3.1 .6 Objective Function . . . . . . . . . . . . . . . . . . 31

3.1 .7 Formulation of the Constraints . . . . . . . . . . . . 31

3.1 .8 Delay Computation . . . . . . . . . . . . . . . . . 33

3.2 Model with Pareto Optimality . . . . . . . . . . . . . . . . 35

$3.2 .1 \quad$ Weighted Sum Optimization . . . . . . . . . . . . 35

3.2 .2 Hierarchical Optimization $\ldots \ldots \ldots \ldots$

3.2 .3 Trade-Off Method . . . . . . . . . . . . . . . . . . . . 36

3.3 Determining the Best Compromise Solution $\ldots \ldots \ldots \ldots$

3.4 The Hypervolume Indicator $\ldots \ldots \ldots \ldots$

3.5 Chapter Summary $\ldots \ldots \ldots \ldots$

$\begin{array}{lll}4 & \text { Results and Analysis } & 40\end{array}$

$4.1 \quad$ Framework for the Fog Planning Problem. . . . . . . . . . . . 40

4.2 Detailed Example $\ldots \ldots \ldots \ldots$

4.3 Results Analysis $\ldots \ldots \ldots \ldots$

$4.3 .1 \quad$ Input Parameters $\ldots \ldots \ldots \ldots \ldots$

4.3 .2 Small Scale Problems . . . . . . . . . . . . . . . . . 56

4.3 .3 Large scale problems . . . . . . . . . . . . . . . . . 65

4.3 .4 Impact of the Number of Possible Placement Location . . . . 72

4.4 Chapter Summary $\ldots \ldots \ldots \ldots \ldots$ 
\begin{tabular}{|lll}
5 & Conclusions and Future Work & 78
\end{tabular}

5.1 Future Works . . . . . . . . . . . . . . . . . . . . . . . . . . . . 79

\begin{tabular}{ll}
\hline List of References & 81
\end{tabular}

\begin{tabular}{|lr|}
\hline Appendix A Results for Small Scale Problem & 88
\end{tabular}

A.1 Instance 1 . . . . . . . . . . . . . . . . . . . . . . . 88

A.2 Instance $2 \ldots \ldots \ldots \ldots$

A.3 Instance $3 \ldots \ldots \ldots \ldots$

A.4 Instance $4 \ldots \ldots \ldots \ldots$

\begin{tabular}{|ll}
\hline Appendix B Results for Large Scale Problem & 92
\end{tabular}

B.1 Instance 1 . . . . . . . . . . . . . . . . . . . . . . . . . 92

B.2 Instance $2 \ldots \ldots \ldots \ldots$

B.3 Instance $3 \ldots \ldots \ldots \ldots$. . . . . . . . . . . . . . . . . . 94

B.4 Instance $4 \ldots \ldots \ldots \ldots$

\begin{tabular}{|ll}
\hline Appendix C Results for Single and Multiobjective & 96
\end{tabular}

C.1 Instance $1 \ldots \ldots \ldots \ldots \ldots$. . . . . . . . . . . . . 96

C.2 Instance $2 \ldots \ldots \ldots \ldots \ldots \ldots \ldots$

C.3 Instance 3 . . . . . . . . . . . . . . . . . . . . . . . . . . 98

C.4 Instance $4 \ldots \ldots \ldots \ldots$ 


\section{List of Tables}

$4.1 \quad$ Fog type features for the planning example . . . . . . . . . . . 47

4.2 Link type features for the planning example $\ldots \ldots \ldots \ldots$

4.3 Topology and optimization input parameters for the example. . . . . 48

4.4 Edge device input parameters for each cluster $\ldots \ldots \ldots \ldots$

4.5 Delay formulation of the planning example $\ldots \ldots \ldots \ldots \ldots$

$4.6 \quad$ Traffic formulation of the planning example $\ldots \ldots \ldots \ldots \ldots$

4.7 Characteristics of the different fog types $\ldots \ldots \ldots \ldots \ldots$

4.8 Characteristics of the different link types . . . . . . . . . . . 54

4.9 The edge device cluster input parameters $\ldots \ldots \ldots \ldots \ldots \ldots$

4.10 The edge device input parameters for each edge device in a cluster. . 55

4.11 Problem size for the small scale FPP . . . . . . . . . . . . . . 57

4.12 Results obtained from the solver for small scale problems (average over

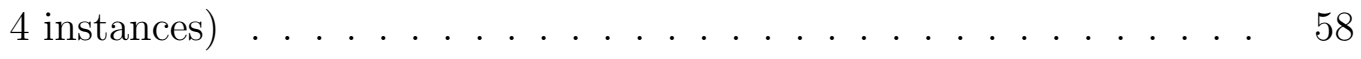

4.13 Solutions comparison among the three methods over 4 instances . . . 62

4.14 Solutions comparison between single objective and multiobjective (over 4 instances $) \ldots \ldots \ldots \ldots$. . . . . . . . . . . . . . 63

4.15 Problem size for the large scale FPP … . . . . . . . . 66

4.16 Results obtained from the solver for large scale problems (average over

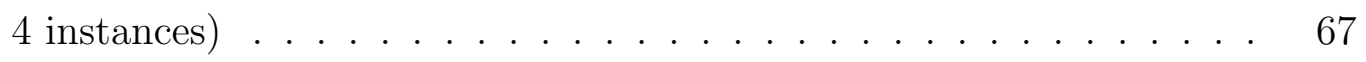

4.17 Average solution comparison among the three methods over 4 instances for large scale problems . . . . . . . . . . . . . . . . . 70

4.18 Solutions comparison between single objective and multiobjective over 4 instances for large scale problems $\ldots \ldots \ldots \ldots 71$

A.1 Result obtained from the solver for small scale problem: Instance 1 . 88

\begin{tabular}{|lll}
\hline A.2 & Result obtained from the solver for small scale problem: Instance 2 & 89
\end{tabular}

A.3 Result obtained from the solver for small scale problem: Instance 3 . 90

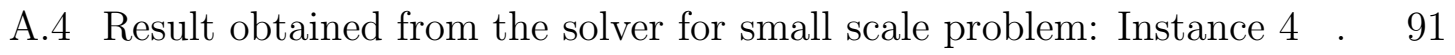


B.1 Result obtained from the solver for large scale problem: Instance 1. . 92

B.2 Result obtained from the solver for large scale problem: Instance 2. . 93

B.3 Result obtained from the solver for large scale problem: Instance 3. . 94

B.4 Result obtained from the solver for large scale problem: Instance 4. . 95

C.1 Result for single objective and multiobjective: Instance 1 . . . . . . 96

C.2 Result for single objective and multiobjective: Instance 2 $\ldots \ldots .99$

C.3 Result for single objective and multiobjective: Instance 3 . . . . . . 98

C.4 Result for single objective and multiobjective: Instance 4 . . . . . . 99 


\section{List of Figures}

$2.1 \quad$ Reference architecture for fog computing $\ldots \ldots \ldots \ldots \ldots \ldots$

3.1 Graphical representation of the notations . . . . . . . . . . 30

3.2 HV enclosed by non-dominated solution $a_{1}, a_{2}, a_{3}$ and $a_{4}[75] \ldots \quad \ldots \quad 39$

4.1 Steps to solve the FPP $\ldots \ldots \ldots \ldots \ldots$

4.2 Steps to solve the FPP with the weighted sum method . . . . . . . 42

4.3 Steps to solve the FPP with the hierarchical method . . . . . . . . 44

4.4 Steps to solve the FPP with the trade-off method . . . . . . . . . . 45

$4.5 \quad$ Edge device locations and potential locations of fog nodes. . . . . . 47

4.6 Optimal solution found by Cplex $\ldots \ldots \ldots \ldots \ldots$

4.7 Delay comparison with 95\% CI for small scale problems (average over 4 instances $) \ldots \ldots \ldots \ldots$

$4.8 \quad$ Traffic comparison with 95\% CI for small scale problems (average over 4 instances $) \ldots \ldots \ldots \ldots$

$4.9 \quad$ Solution time comparison for small scale problems (average over 4 in-

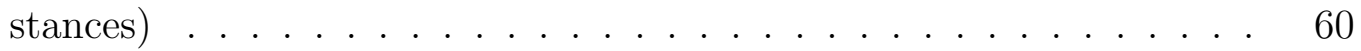

4.10 Average HV indicator for small scale problems . . . . . . . . . . 65

4.11 Delay comparison with $95 \%$ CI for large scale problems (average over

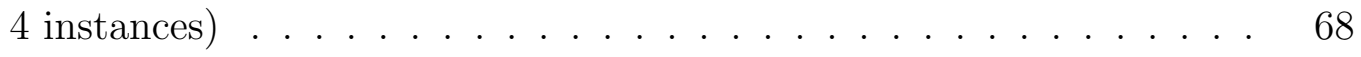

4.12 Traffic comparison with 95\% CI for large scale problems (average over

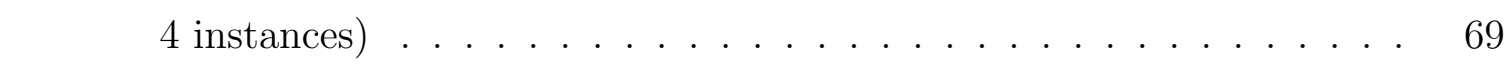

4.13 Solution time comparison for large scale problems (average over 4 instances $) \ldots \ldots \ldots \ldots$

4.14 Average HV indicator for large scale problems . . . . . . . . . . 72

4.15 Delay comparison for different number of possible locations using the weighted sum method . . . . . . . . . . . . . . . . . . 73

4.16 Delay comparison for different number of possible locations using the hierarchical method . . . . . . . . . . . . . . . . . . . . 73 
4.17 Delay comparison for different number of possible locations using the trade-off method . . . . . . . . . . . . . . . . . . . 73

4.18 Traffic comparison for different number of possible locations using the weighted sum method. . . . . . . . . . . . . . . . . . . . . . . 74

4.19 Traffic comparison for different number of possible locations using the hierarchical method . . . . . . . . . . . . . . . . . . 74

4.20 Traffic comparison for different number of possible locations using the trade-off method . . . . . . . . . . . . . . . . . . . . . . 75

4.21 Solution time comparison for different number of possible locations using the weighted sum method . . . . . . . . . . . . . . . . . . 75

4.22 Solution time comparison for different number of possible locations using the hierarchical method . . . . . . . . . . . . . . . . . . . . 75

4.23 Solution time comparison for different number of possible locations

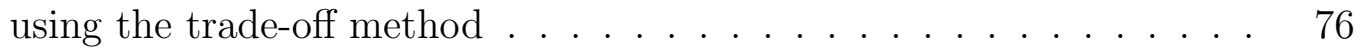




\title{
List of Abbreviations
}

\author{
AHP Analytic Hierarchy Process \\ API Application Program Interface \\ APN Access Point Name \\ AWS Amazon Web Services \\ B\&B Branch and Bound \\ BS Base Station \\ CEP Complex Event Processing \\ CI Confidence Interval \\ CoT Cloud of Things \\ DC Data Center \\ EC2 Elastic Compute Cloud \\ ECG Electrocardiogram \\ ETC Electronic Toll Collection \\ FPP Fog Planning Problem \\ GCE Google Compute Engine \\ GH Greedy Heuristic \\ GPON Gigabit Passive Optical Network \\ HTML Hypertext Markup Language \\ HTTP Hypertext Transfer Protocol
}




$\begin{array}{ll}\text { HV } & \text { Hypervolume } \\ \text { IaaS } & \text { Infrastructure-as-a-Service } \\ \text { IETF } & \text { Internet Engineering Task Force } \\ \text { IoT } & \text { Internet of Things } \\ \text { IP } & \text { Internet Protocol } \\ \text { IT } & \text { Information Technology } \\ \text { MCC } & \text { Minimum Correlation Coefficient } \\ \text { MDC } & \text { Micro Data Center } \\ \text { MEC } & \text { Mobile Edge Computing } \\ \text { MILP } & \text { Mixed Integer Linear Program } \\ \text { nDC } & \text { Nano Data Center } \\ \text { NIST } & \text { National Institute of Standards and Technology } \\ \text { NP } & \text { Nondeterministic Polynomial } \\ \text { OPL } & \text { Optimization Programming Language } \\ \text { OR } & \text { Operation Research } \\ \text { PaaS } & \text { Platform-as-a-Service } \\ \text { PKI } & \text { Public Key Infrastructure } \\ \text { PM } & \text { Physical Machine } \\ \text { QoS } & \text { Quality of Service } \\ \text { RAN } & \text { Radio Access Network } \\ \text { RFID } & \text { Radio-frequency Identification } \\ \text { SaaS } & \text { Software-as-a-Service } \\ \text { SDN } & \text { Software Defined Network } \\ \text { SLA } & \text { Service Level Agreement } \\ \end{array}$


TEE Trusted Execution Environment

V2V Vehicle-to-Vehicle

vCPU Virtual Central Processing Unit

VM Virtual Machine

WDM Wavelength Division Multiplexing 


\section{Chapter 1}

\section{Introduction}

Cloud computing is a computing paradigm, where infrastructure of dynamic scalability is provided for data, file storage and applications. Cloud computing is used by a large pool of connected private or public networks. With massive storage and computation capacity, cloud computing has been a driving force in Internet of Things (IoT) providing a variety of services. With the evolution of machine learning applications, big data and the increased number of connected devices, the cloud model alone is not applicable for time-critical operations. Issues of performance, security, reliability and high consumption of bandwidth in the cloud promoted the idea of fog computing.

Fog computing can be defined as a non-trivial extension of the cloud residing on the edge of the network. Fog computing provides the same services as the cloud in a limited or full service. It is a Micro Data Center (MDC) focused on applications and services which are broadly distributed. An MDC is a small, containerized Data Center (DC). Unlike the traditional cloud DC, an MDC can be as small as a19 inch box with less than 10 servers and 100 Virtual Machines (VM) [1]. Fog computing is a highly virtualized architecture providing services like computation, storage and networking between end-devices in an IoT and Cloud of Things (CoT). Unlike the centralized technology of cloud, fog computing is targeted towards widely distributed applications and services. Fog applications have a distributed directory system to communicate with mobile devices acknowledging mobility techniques. Fog computing supports low latency applications such as augmented reality, gaming, video streaming, etc. The widespread geographical distribution of fog nodes, defined as the location where several physical devices provide resources such as virtual Central Processing Unit (vCPU), memory and storage to various services [2], will deliver high 
quality streaming services to mobile nodes and moving vehicles through proxies and access points positioned in vital places such as tracks and highways. The cloud will always have an important role in IoT. Hence, by providing an improved Quality of Service (QoS) and reducing the amount of traffic in the cloud, fog computing is not a replacement but a complement to the cloud architecture.

\subsection{Problem Statement}

With the complexity of networks and the large number of devices, service providers need efficient planning tools to design optimal networks. Efficient network planning tools can help in designing optimal networks guaranteeing better service to the end users. The fundamental goal of network planning is to implement an optimum network addressing realistic traffic parameters. Unlike earlier generations, computerbased automatic planning tools are now required for large networks. The network design problem can be solved by using simulation or solving mathematical models. Simulation is typically used when a mathematical model cannot be developed for a network design problem. A basic representation of the network is modeled in a tool where the simulated network is examined with different inputs and parameters. Unfortunately, most network planning problems are NP-hard [3,4].

Network planning is a massive research field with many applications in IP networks, optical networks, wireless networks, cellular networks, cloud computing, etc. To the best of our knowledge, no work has considered the planning and design of fog networks in collaboration with the cloud. It can be recognized that the fog nodes will be the distributed fog network entities enabling the deployment of fog services. For a normal fog network, there will be a lot of fog nodes to be installed and to deliver the expected performance improvements, proper planning of these fog nodes is necessary. Consequently, many factors such as the number of nodes, locations, node sizing, connectivity between fog and cloud, traffic distribution and so on need to be considered. Given the large number of fog nodes in the network combined with a vast number of edge devices over the entirety of the geographical region of interest, the search space for an optimal solution is huge. Therefore, sophisticated methods and algorithms are required to help network planners in the decision making process.

The purpose of the fog network is to improve the end user experience by reducing parameters like delay in the network, the amount of traffic in the cloud and so 
on. Optimizing more than one parameters converts the problem into a multiobjective optimization problem resulting in a number of non-dominated solutions. The multiobjective optimization problem can be studied from different viewpoints with different solution goals while solving it. Hence, it is very hard to find a best compromised solution for all the objective functions even with a comprehensive knowledge of the problem.

There are different methods to solve multiobjective optimization problems. However, it is a very complex task to choose the best method as different methods have various requirements and can perform differently with different problems. Ideally, for the best convergence towards the Pareto set, the optimization method that handles the minimum amount of complexity should be used [5,6]. But defining the amount of complexity is not straightforward. To mitigate that, a number of methods can be used simultaneously to understand and solve the problem. Moreover, the planning problem can be solved with exact and approximate multiobjective algorithms, each having its own advantages and disadvantages.

The fog network planning is a complex but necessary step towards building efficient fog networks. To that end, this thesis addresses the above mentioned problems and proposes an exact mathematical model with different multiobjective optimization methods for the planning and design of fog networks.

\section{$1.2 \quad$ Research Objectives}

According to the problem statement explained in the previous section, the main objective of the thesis is to develop a tool to help network providers with the planning and design of fog networks. More precisely, we aim to achieve the following two subobjectives:

- Propose a mathematical model for the planning and design of fog networks from a green field scenario. The model will simultaneously determine the optimal location, the number, and the capacity of the fog node(s) and the interconnection between the installed fog node(s) and the cloud while considering edge device requests. The goal of the model is to minimize the delay in the network and the amount of traffic going to the cloud.

- Evaluate the performance of the proposed model and analyze the results with different optimization methods. The three multiobjective optimization methods 
are the weighted sum, the hierarchical and the trade-off method. The goal of this sub-objective is to compare the three multiobjective optimization methods and find the best method for appropriate scenarios.

\subsection{Methodology}

To achieve our objectives, we will first review the different concept of cloud computing. This will help in realizing the weakness of the current cloud architecture and the need of fog computing for IoT. The current contributions in fog computing will also be reviewed to have an updated view of the state-of-the-art. The following methodology will be used in approaching the planning problem of fog networks.

- Generate a snapshot of traffic with realistic parameters: The requested amount of traffic needs to be known to properly plan fog networks. To generate the traffic, a small program will be developed taking into consideration different aspects like number of packets, amount of memory/vCPU requested, etc.

- Develop the mathematical model: In order to develop the mathematical model, a set of mathematical equations will be written to represent the architecture of fog networks. The goal of the model will be to minimize the delay experienced by the users and minimize the amount of traffic that must go to the cloud. Different assignment, capacity and uniqueness constraints will be taken into consideration to create a realistic fog network scenario. A set of decision variables will be used in determining the type and number of fog node(s) to be placed in the network. Since we will cast the problem as a multiobjective optimization problem, a multiobjective Pareto-optimization approach will be adapted to find the optimal fog network deployment that considers both objective functions. The model will be written in OPL and a solver called CPLEX will be used to solve the problem. The solver will return the optimal solution or the best solution found when it reaches its time limit.

- Analyze the results of the fog planning problem: A detailed example will be used to explain the planning problem. Then, using CPLEX, we will solve the planning problem for the three methods with different problem sizes. The result analysis will investigate the comparison of the three optimization methods with 
respect to traffic, delay and solution time. Next, the Pareto-efficiency of the three methods will be analyzed for each problem size.

\subsection{Thesis Contributions}

The main contribution of this thesis is to to develop an exact model for the planning and design of fog networks. The major contributions of this thesis are summarized as follows:

- A mathematical model is proposed for the planning and design of fog networks for a brand new infrastructure in current cloud architecture. The main contribution of the model is the consideration of realistic edge device request parameters like vCPU, memory, etc. in determining the optimal location, capacity, type and number of fog node(s). Moreover, the optimal interconnection between the fog node(s) and the cloud is also determined by the planning model. As the model considers the delay in the network and the traffic going to the cloud, the planning tool will help fog service providers to deploy fog node(s) in the existing cloud architecture in an efficient manner with an improved QoS.

- As there are two objective functions, the main intention is to study different multiobjective optimization techniques to address the Pareto optimality and trade-off between the objective functions. As a result, three multiobjective optimization methods are used in solving the fog planning problem. Then, the results are analyzed and compared for the three methods with the weighted sum method achieving the best trade-off results for the two objective functions.

\subsection{Thesis overview}

The rest of the thesis is organized as follows. In Chapter 2 , a literature review of cloud and fog computing is conducted. Chapter 3 introduces the planning problem followed by the mathematical model to solve the fog planning problem. Then, the mathematical model is modified to the three multiobjective optimization models. Chapter 4 presents the simulation results for the three modified models compared in terms of objective functions, solution time and Pareto-efficiency. Finally, the thesis 
is concluded in Chapter 5 by summarizing the findings and proposing future research directions. 


\section{Chapter 2}

\section{Background and Related Work}

Since the concept of fog computing is an extension of cloud computing, it is important to understand different notions related to cloud computing. To that end, Section 2.1 first discusses the history and the current architecture of cloud computing and then introduces the concept of fog computing. Section 2.2 reviews some of the applications and current research specifically related to fog computing. Section 2.3 reviews the network planning and design process and finally we summarize the chapter in Section 2.4.

\subsection{Introduction to Cloud Computing}

Cloud computing is experiencing an exponential growth over the years. As the cloud service enables new and efficient business models, small and medium businesses are integrating to the cloud platform. The word cloud was commonly used as a metaphor for Internet and a network on telephone schematics was usually illustrated with a standardized cloud like shape. Later, it was used to label the Internet in computer network diagrams. Sometime around 1955, John McCarthy came upon with the time sharing theory, which is very similar to present day cloud computing. In 1970s, IBM released an operating system called VM that allowed users to have more than one virtual systems (or VMs) on a single physical node. The 50s time sharing model was taken to a whole new level by the VM operating system and many of the elemental functions in virtualization software that are presently used can be traced back to this early VM operating system. To provide more users through shared connection to the same physical infrastructure, in 1990s, virtualized private network connections were offered by telecommunication companies. This development facilitated a better 
network equity and higher control over bandwidth usage by adjusting the traffic as required. In the meantime, with the fervent start in virtualization for PC-based systems, and with the availability of Internet,the integration of virtualization was the next logical step. In 1997, Professor Ramnath Chellappa coined the term cloud computing in a talk on new computing paradigm. In 2002, with the creation of Amazon Web Services (AWS), Amazon provided an advanced system of cloud services accommodating storage to computation. Later, in 2006, the Elastic Compute Cloud (EC2) was introduced by Amazon as a commercial web service. The EC2 enabled renting of computers by small companies on which their own computer applications could be run. In 2009, the Google App Engine brought low-cost computing and storage services, and Microsoft followed with Azure.

\subsubsection{Definitions}

Although cloud computing is widely acknowledged, many researchers suggested their own definition with respect to their research domain. The National Institute of Standards and Technology (NIST) defines cloud computing as a model for enabling ubiquitous, convenient, on-demand network access to a shared pool of configurable computing resources (e.g., networks, servers, storage, applications and services) that can be rapidly provisioned and released with minimal management effort or service provider interaction [7]. Sotomayor et al. denote that cloud is more often used to refer to the IT infrastructure deployed on an infrastructure as a service provider data center [8]. Vasquero et al. mention that clouds are a large pool of easily usable and accessible virtualized resources (such as hardware, development platforms and/or services). These resources can be dynamically reconfigured to adjust to a variable load (scale), allowing also for an optimum resource utilization. This pool of resources is typically exploited by a pay as you go model in which guarantees are offered by the infrastructure provider by means of customized Service Level Agreement (SLA) [9]. As can be seen, it is hard to come up with a single definition of cloud computing. In fact, cloud computing is not a new technology, but rather brings together a number of standing technologies with a new operational model to run business in a new dimension. Certainly, many cloud computing technologies, such as virtualized environments and utility computing, are not new. Instead, these existing technologies

are leveraged to fulfill the economic and technological demand required by today's network environment. [7]. 


\subsubsection{Related Technologies}

Cloud Computing emerged from years of research in various similar technologies. The following technologies are usually compared with cloud computing, each sharing some similarities with cloud computing.

\section{Grid Computing}

Grid computing is a model of distributed computing where computing, data, storage, application or network resources are coordinated and shared over dynamic and geographically dispersed organization $[10]$. There are some similar features between cloud computer and grid computer such as:

- Multitask and multitenancy are part of both computing technologies, implying that customers can perform various tasks, gaining access to single or multiple application instances. Peak load capacity and infrastructure costs can be reduced by sharing resources among a large number of users. Cloud and grid computing administer SLAs for guaranteed uptime availability, for instance, 99 percent. When the service declines below the level of guaranteed up time service, service credit is offered to the consumer for receiving late data.

- Scalability is another common feature between cloud and grid computing. Scalability is achieved through load balancing of application instances connected through web services and running separately on a variety of operating systems. CPU and network bandwidth are allotted and de-allotted on demand. Based on the number of users, instances, and the volume of data transferred at a particular time, the systems storage capacity fluctuates 11.

Similarly, the major differences between grid and cloud are as follows:

- Business Models Business models in grid computing are generally based on mutual agreements between the service provider and the consumer, whereas provision of resources in cloud computing constitutes of more differentiated business models ranging from service provider to service provider and mixed approach.

- Resource Management: While grids depend on batch systems, clouds resource management solution is represented by the application of virtualization technologies. 
- Resource Provision Model: The communications in grid resource provision models are established offline relying on virtual organizations. On the otherside, the usage of SLAs, trust management and compliance are the key to resource provision models in clouds.

- Resource Availability: Sometimes, there are plenty of resources in grids which are idle and at some other times, there are no available resources, therefore the resources are shared on the best-effort delivery. The matter of concern in clouds are to find the balance between utilization of resources to facilitate productive consumption of resources and reducing energy consumption on the one hand and wasting resources due to the standby mode of devices and virtualization overhead on the other.

\section{Utility Computing}

Utility computing comprises the on demand basis of renting computer resources such as network bandwidth, hardware and software as-required [12]. There is a similarity between utility and cloud computing as both concepts entertain the idea of leasing computer technology. But they have some major differences between them. One of the fundamental differences between utility and cloud computing relates to the behavior of the leasing. While a third party is used for software and infrastructure for both technology, these services are much more directly accessed by utility computing. This form of computing makes the use of technology like another utility, such as gas or electricity, and by the end of the month, businesses would be charged according to the usage. Though, all the services in cloud computing are still rented, the source of the services are less known by the companies. Users still pay for what they use, but the company providing the service utilizes a much more complex system of infrastructure and software, usually involving a grid network that supports multiple tasks at once. Another difference is that utility computing is dependent on basic computing practices, often utilizing traditional programming styles in a well-established business context. Cloud computing, in contrast, involves creating an entirely distinctive virtual computing environment that empowers programmers and developers in new ways. 


\section{Autonomic Computing}

Autonomic computing was introduced by IBM as a term to describe a selfmanaging system that tries to get involved in computing system. IBM divided the selfmanaging system into four categories: self-optimizing, self-healing, self-configuring, and self-protecting [13]. The main difference between autonomic and cloud computing is that autonomic computing is focused on reducing the system complexity. Whereas, the objective of cloud computing focuses on decreasing the resource cost rather than to diminish system complexity. As there will be a lot of pre-processing done in the fog nodes, the reduction of resource usage in the cloud will help in focusing to a more automated system.

\section{Virtualization}

Virtualization is a technology providing virtualized resources for high-level applications by abstracting away the specifics of physical hardware. The common name of a virtualized server is virtual machine or VM [7]. Virtualization abstracts compute resources as VMs with association of storage and networking connectivity. The cloud determines how those virtualized resources are allocated, delivered and presented. Virtualization facilitates rapid scaling of resources which is hard to do by non-virtualized environments.

\subsubsection{Service Models}

Cloud services can be classified into three distinct models: Infrastructure-as-aService (IaaS), Platform-as-a-Service (PaaS) and Software-as-a-Service (SaaS). Each one is described below.

\section{Infrastructure-as-a-Service}

IaaS is a vastly automated and standardized service where computer resources are hosted and owned by service providers and provided to customers on-demand, integrating storage and networking capabilities. This infrastructure can be selfprovisioned by customers, using a Web-based graphical user interface that accommodates the overall environment as an IT operations management console [14.

Typical examples of IaaS include: Joyent, Google Compute Engine (GCE), Microsoft Azure, Cisco Metapod, Amazon Web Services (AWS) and IBM SoftLayer. 
Common use-case: Expands present data center infrastructure for temporary workloads (e.g. increased Canada Day holiday site traffic).

\section{Platform-as-a-Service}

PaaS allows developers to build applications and services over the Internet by providing a platform and environment. PaaS services being hosted in the cloud can easily be accessed by users via web browsers. Users can create software applications with the help of tools supplied by the provider of the service. In PaaS, customers can subscribe to preconfigured features, giving the option to include their required features or discarding the unwanted ones [15].

Typical examples of PaaS include: Google App Engine, Etelos, Force.com, IBM Bluemix, Qrimp and AppJet.

Common use-case: Increases developer efficiency and work rates while also minimizing an applications time-to-market.

\section{Software-as-a-Service}

SaaS is the cloud-based delivery of complete software applications that run on infrastructure managed by the vendor. SaaS applications use the web to deliver applications whose interface is accessed on the clients side and handled by a thirdparty. Most SaaS applications can be run directly from a web browser without the requirement of downloads or installation, although some require plugins [16.

Typical examples include Cisco WebEx, Citrix GoToMeeting, Workday, Google Apps, Salesforce.

Common use-case: Replaces conventional on-device software.

\subsubsection{Deployment Models}

\section{Public Cloud}

Public clouds provide users with a lucrative pay-as-you-go model. Public clouds are owned and managed by third parties. The infrastructure costs are divided among a number of different users delivering remarkable economies of scale to customers. The same infrastructure is used by all customers with finite configuration and security protections operated and supported by the provider. Public clouds have the capability to scale smoothly, as they can be larger than enterprise clouds, if required. 


\section{Private Cloud}

Private clouds are solely built for a single company. Private clouds are intended to acknowledge their interest on data security. Unlike public clouds, there is a better authority on security in private clouds. There are two different type of private clouds:

- On-premise private clouds: On-premise private clouds, also called internal clouds, are accommodated within the company, hosting its own data center. This cloud computing is best fitted for applications requiring complete management and configurability of the infrastructure and security by providing a more regulated protection. The capital and operational costs are incurred by the company itself.

- Externally hosted private clouds: In this type of private cloud, an exclusive cloud environment with maximum privacy is provided to the enterprise. The cloud provider hosts the cloud externally especially for the companies that avoid public cloud.

\section{Hybrid Cloud}

Hybrid clouds are the combination of both public and private clouds. Hybrid clouds improve the flexibility of computing, where infrastructures of third party can be used partially or fully by the cloud providers $[17$. The hybrid cloud environment is capable of providing on-demand externally provisioned scale. Any unforeseen rise in workload can be negotiated by reinforcing a private cloud with the available infrastructure of public cloud.

\subsubsection{VM Placement in Cloud}

Similar to fog nodes in a fog network, the VM is one of the key entities in the cloud computing architecture. The planning of VM is one of the most challenging problem which aims to find the best PM to host the VMs. The VM placement and assignment has an effect on the overall resource utilization, performance, power consumption and cost reduction of data centers. With a massive number of possible optimization criteria, a number of formulations have been proposed over the years for the static and dynamic VM placement problem. For example, a stable traffic aware VM placement problem is studied in [18]. The authors defined the problem as Min Cut 
Ratio-aware VM Placement (MCRVMP) and propose two heuristics called 2-Phase Connected Component-based Recursive Split (2PCCRS) and Greedy Heuristic (GH). The 2PCCRS adopts a two-stage approach and solves the problem with the aid of a mathematical solver. The first stage places the connected components on network switches whereas in the second step, the VMs are placed on PM. In GH, the VMs are placed greedily on the available hosts without using any mathematical programming. The results show that there is an increase in data center scalability while decreasing the number of dropped packets. A hierarchical energy-aware resource management solution is proposed in [19]. The resource demands are modeled as random variables considering the correlation among the variables. Compare to the non-hierarchical model, the hierarchical model achieves around 15\% energy cost reduction. The authors in [20] propose a Mixed Integer Linear Program (MILP) formulation that aims to place the VMs in the data center with minimum power consumption. The objective function is to minimize the sum of the power consumption of all associated DCs, the power consumption in the Internet Protocol (IP) layer and the power consumption in the Wavelength Division Multiplexing (WDM) layer. The results show a reasonable amount of power saving in geographically distributed multiple medium size DCs.

One of the most common approaches for the optimization is multi-objective solved as mono-objective. The authors in 21] adopt a variation of the weighted sum method in optimizing virtual machine placement and traffic flow routing. The joint optimization demonstrates a reduction in network power costs in cloud data centers. In 22 , the authors propose a VM placement scheme minimizing the number of active physical servers and network elements. The results are an optimized network traffic and a reduction in energy consumption. Static and dynamic server consolidations are proposed in 23] for the VM migration. For the dynamic consolidation, the authors adopt a hierarchical approach by introducing the previous mapping of VM in physical servers as a constraint. Initially starting with a zero mapping, after each iteration the previous mapping is added. An energy efficient approach based on the Minimum Correlation Coefficient (MCC) method for VM placement is proposed in 24. A fuzzy Analytic Hierarchy Process (AHP) is used to make a trade-off between SLA and low energy. A suitable trade-off between SLA violation reduction and power efficiency in cloud data centers was evaluated from the simulation results. 


\subsubsection{Limitations}

Though cloud computing comes with lot of advantages, a rapid growth of users and the demand of higher QoS results in following limitations.

- Performance: Cloud computing is established within the Internet, a large nonhomogeneous network with attributes of numerous technologies, speeds, topologies with non-central control. Due to the heterogeneity and loosely controlled nature of Internet, there is a lot of performance related challenges in the cloud. One such issue that affects the performance is latency. Users who are located far away from cloud providers can experience high delays.

- Security and privacy: One of the biggest limitations of the cloud is security and privacy. When customer data is outside the firewall, the integrity and confidentiality of data is highly vulnerable. As the system responses and data transmission travel a long distance to and from the user device to cloud, different types of encryption are required. As cloud providers use diverse structures and independent locations in delivering the technology, there is less trust between customers and providers.

- Bandwidth cost: The bandwidth cost for data-intensive applications is very high. Although cloud computing reduces expenses on hardware and software, companies have to spend more on network bandwidth.

- Reliability: Cloud computing can cause significant time delays to companies resulting in financial losses due to network failures and outages. Moreover, when all the raw data generated by end devices is sent at the same time, it could create a bottleneck. Bottlenecks from high volume of data transfers are one of the most common cause of network crash and outages 25 .

\section{$2.2 \quad$ Fog Computing}

To address some of the limitations mentioned previously, the concept of fog computing has been recently introduced. Fog computing is a flexible system of connectivity which brings the computational, storage and networking elements of the cloud near to the edge of the network. Fog computing can have an impact on the performance, security, bandwidth and reliability as described below: 
- Performance: One of the mission critical applications in IoT will be tactile Internet which requires extremely low latency, high reliability and security with real time data processing. By performing the required processing near the devices, fog computing can provide real time response with reduced latency.

- Security and privacy: As fog computing is not centralized and it is closer to the edge devices, it enhances the security of encrypted data making it less vulnerable to hostile elements. This will allow the users to have their data and applications in close proximity, building more trust between the users and service providers. As the cloud is located in a remote location, the nearest cloud DC can be outside the country border. If a company wants to keep its data within the country, the fog can be a viable option compared to the remote cloud.

- Bandwidth cost: Instead of using the backbone network, users can download through local connections which can reduce the bandwidth cost significantly. Moreover, less data will be sent to the cloud since processing will be done locally.

- Reliability: With the help of fog localization and cloud centralization, there can be interplay of data processing between fog and cloud, hence reducing the burden of all the data processing in the cloud.

\subsubsection{Architecture}

Figure 2.1 shows a reference fog architecture consisting of different layers. The bottom-most layer comprises of end devices, gateways and sensors. Additional applications are installed for efficient use of the technology. The second layer is where the fog nodes are resided along with the core network. The last layer consists of cloud components. Below is a description of the three layers.

\section{Layer 1}

The first layer is composed of smart objects (IoT devices) that are generating data. The devices can be mobile phones, wireless sensors, vehicles, etc. with various applications installed transmitting and receiving data and information to the immediate upper layer and/or the cloud layer. 


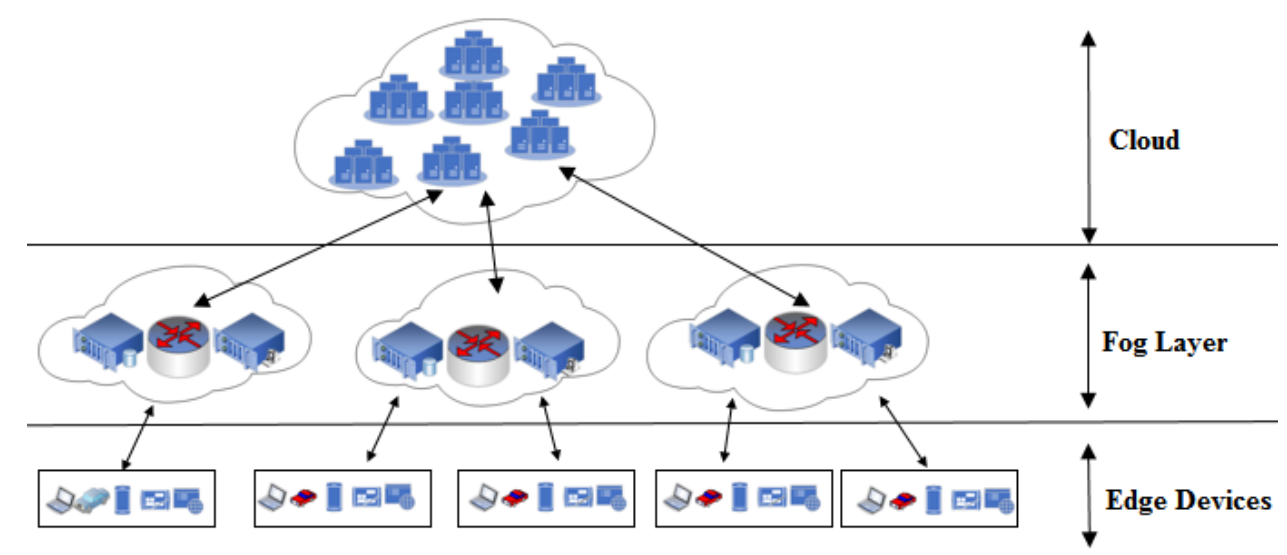

Figure 2.1: Reference architecture for fog computing

\section{Layer 2}

The fog computing layer is made up of fog nodes and smart devices such as intelligent gateways, routers and Access Point Name (APN). In this layer, the fog nodes and smart devices perform tasks like routing, data storing and preprocessing, caching and packet forwarding towards the cloud. Additionally, this layer incorporates resource management software while maintaining the entire infrastructure. This layer ensures the QoS to the different fog technology applications as well as the connectivity between the fog nodes and the cloud.

\section{Layer 3}

The cloud computing layer consists of servers and data centers with extensive storage capability. Applications and data which require permanent storage and powerful processing are redirected to the cloud. Unlike traditional cloud architecture, the cloud does not serve every individual query. The fog computing layer decides the accessibility and usage of the cloud in an efficient and disciplined manner.

\subsubsection{Protocol}

There is no implementation or suggestion of any specific protocol for fog computing. Since fog nodes will be providing real time service to numerous edge devices, it is imperative to look at the protocols with more details for a relaiable service. As fog nodes are nested in a middle layer, a number of IoT protocols, as mentioned below, 
which are currently being developed or in use can be applied.

\section{Data Distribution System}

DDS is a middleware protocol and Application Program Interface (API) standard used for data-centric connectivity from the Object Management Group. DDS provides low-latency data connectivity and a scalable architecture making it useful for missioncritical IoT applications. It uses a publish/subscribe model making sure that data of one node is known by all other nodes 26 .

\section{Constrained Application Protocol}

CoAP is a low cost software protocol designed to be used in small, low-power wireless sensor networks, switches and similar components that are controlled remotely. CoAP is designed by the Internet Engineering Task Force (IETF) and the interface is modeled after Internet Hypertext Transfer Protocol (HTTP) requests. CoAP provides low overhead and multicast support making it an efficient protocol 27.

\section{Message Queue Telemetry Transport}

MQTT was invented by Dr. Andy Stanford-Clark of IBM, and Arlen Nipper of Arcom in 1999. It is a publish-subscribe-based messaging protocol suitable for constrained devices and high-latency, low-bandwidth or unreliable networks. There are different modifications of MQTT such as IBM MessageSight, Mosquitto, emqttd, RabbitMQ, HBMQTT - which differ in features and may employ additional features upon requirement 28.

\section{Advanced Message Queuing Protocol}

AMQP is an open standard application layer protocol which provides interoperability between servers. It is a message-oriented middleware with a robust system guaranteeing endpoint to endpoint message delivery despite node failures. Despite being heavyweight and more established compared to other protocols, it can be used in the IoT framework where message reliability is the main focus [29]. 


\section{Alljoyn}

Alljoyn is a collaborative open source software through which compatible devices and applications communicate together [30. It is a flexible framework promoting proximal network. Although the communication layer is limited to wi-fi, it can be used in fog network.

\subsubsection{Application}

The concept of fog computing has many different important applications. Below we review these applications and cite relevant work where fog is playing an important role.

\section{Vehicular Networks and Smart Grid}

The authors in [31] propose mobile fog, which is geospatially distributed, largescale and latency sensitive on demand fog service for future Internet applications. Two large scale vehicle-to-vehicle (V2V) application scenarios are simulated. The first application is V2V video streaming and the second is mobility driven Complex Event Processing (CEP), where sensor data is requested for processing from a certain query range by a vehicle. The simulation results show that compare to cloud computing, the fog computing approach reduces network traffic and latency. A literature review by the author in [32] discusses the application of the fog computing framework to implement Software Defined Network (SDN) for vehicular networks. This framework may help in efficient traffic light control and accommodate a number of inputs like Electronic Toll Collection (ETC), Radio-frequency Identification (RFID) tags in cars by installing readers in traffic lights, cameras at traffic lights, etc. creating green traffic waves. Furthermore, the author proposes a multi-layered demand response management system connected to fog devices in a smart grid. Effective coalition

between consumers, fog and cloud can minimize the overall power loss as well as reduce communication costs within a smart grid.

\section{Healthcare}

The authors in [33] propose a three-layer architecture integrating a role model, a layered cloud computing architecture, as well as a fog-computing-informed paradigm to provide a feasible architecture for healthcare and elderly-care applications. The 
proposed model employs a service-oriented architecture for device mapping where the process flow of the healthcare application is modeled using Business Process Modeling Notation (BPMN). The fog computing layer improves the performance of the whole system by providing computing and storage, mobility support, security measures, location awareness and low latency. The validity of the architectural model was exhibited by a use-case as a template for a smart sensor-based healthcare infrastructure. In [34], FAST, a fog computing assisted distributed analytic system to monitor fall for stroke mitigation is proposed. A set of new fall detection algorithms was developed by the authors based on acceleration magnitude values and non-linear time series analysis techniques, as well as new filtering techniques to facilitate the fall detection process. Fog computing was used to design and employ a real-time fall detection system distributing the analytic throughout the network by dividing the detection between the edge devices and servers in cloud. The proposed system attains the high specificity and sensitivity when investigated with real-world data. Moreover, the response time and energy consumption of the system are similar to the most efficient current approaches. In [35], the authors conduct a case study on Electrocardiogram (ECG) feature extraction using fog computing at smart gateways. The fog layer improved on bandwidth utilization, QoS assurance and emergency notification. The experimental results show that more than $90 \%$ bandwidth efficiency and low-latency real time response could be achieved using fog computing.

\section{Preprocessing and Content Delivery}

The integration of IoT with cloud computing can be one of the primary use of fog computing. However, there are many challenges in this integration. The authors in [36] discuss one of the major challenges which is data trimming or preprocessing. There will be congestion in data center and core network when sending immense amount of raw data generated by IoT environments. A smart gateway-based communication is proposed for trimming or preprocessing data before sending it to the cloud. The IoT devices can send their generated data by using single-hop or multi-hop communication. In single-hop, the data goes directly to the smart gateway for necessary preprocessing whereas in the multi-hop, the data will go through the sink node to the smart gateway. The smart gateway with the help of fog computing can provide a better utilization of network and cloud resources. In [37], the authors discuss the use of edge servers in the fog computing architecture for web optimization. Users will be 
connected to the Internet through fog nodes. Each HTTP request made by the user will go through the fog nodes where the fog nodes will reduce the time for web page to load by performing different optimization. General web optimizations like smartly organizing the web page composition, caching Hypertext Markup Language (HTML) components and minimizing the size of web objects can be done by the fog devices. In addition to that, the fog devices will perform optimizations on the basis of network conditions and user behavior. Moreover, the user machines can be monitored by fog devices and it can send graphics of suitable resolutions depending on the browser rendering time.

\subsubsection{Current Research Related to Fog Computing}

There are many organizations and academies focusing their research on fog computing. The open fog consortium is founded by members from Cisco, Dell, Intel, Microsoft, ARM and Princeton University [38. It is working on influencing standards development through liaisons with other organizations as well as promoting innovation and industry interest in fog computing. In this section, we outline the current and future research directions in fog computing.

\section{Programmability}

Although the mobile fog in [31] is a fundamental development in fog and named mobile, the model is based on a tree topology where the fog nodes are fixed. Hence, a more general model may need to be proposed with diverse networks where fog nodes are decentralized and dynamically mobile. There are plenty of active research in computation offloading in the mobile and cloud computing domain [39,40]. The important question in offloading in fog computing is how to accord with the three fold dynamic of a) radio access, b) nodes in the network and c) resources associated to it. A context adaptive model for offloading in Mobile Edge Computing (MEC) programming framework is presented in [41] named as Cloudaware. The authors present the offloading framework with the objectives of i) speed up computation, ii) save energy or bandwidth and iii) enable offloading for dynamic mobility. Further studies should be done in learning how to offload in the multi-layered device-fog-cloud infrastructures. 


\section{Energy Consumption}

Since fog computing is a distributed technology with a large number of nodes, it can be less energy-efficient compared to the centralized cloud computing. In [42], the authors compare the energy consumption of applications using nano data centers (nDCs) in fog computing and using centralized DCs in cloud computing. The authors proposed time-based and flow-based energy consumption model. A set of experiments and measurements were performed to validate the models. The findings suggest that depending on several system design factors, the nDCs might result in energy saving. However, the energy savings are limited to infrequently accessed applications that generate and distribute large amount of data in end-user premises. Hence, the reduction of energy consumption in fog computing still need to be addressed. The authors in [43] study the tradeoff between power consumption and delay by mathematically formulating the workload allocation problem in fog-cloud computing system. Simulation and numerical results show that communication bandwidth and transmission latency can be reduced by drawing slightly more energy consumption.

\section{Security and Reliability}

Similar to current virtualized environments, fog computing is challenged with security and privacy issues. Authentication at different level of fog nodes is one of the major security issues identified by the authors in 44]. Public Key Infrastructure (PKI) involving multicast authentication [45] can be a solution to the problem. Authentication cost can be reduced by using measurement-based methods to identify unwanted fog nodes. Another promising solution for authentication can be the Trusted Execution Environment (TEE) technique [46]. The policy management issues in a fog environment were identified by the authors in [47], and a policy-based management framework and policy specification criteria is proposed. The efficiency of the proposed framework was highlighted by suggesting a set of use-case scenarios based on smart transportation systems.

\section{Resource Management and Accountability}

The authors in [48] investigate the optimization of maximum task completion time minimization problem in fog computing supported software-defined embedded system 
(FC-SDES) with joint consideration of image placement and task scheduling. A lowcomplexity three-stage algorithm is proposed dealing with $\mathrm{I} / \mathrm{O}$ and task computation time. The efficiency of the algorithm was validated by performance evaluation from the simulation based studies. A service oriented resource management model for fog deployment is presented by the authors in [49, which allow IoT devices to operate efficiently with fair management of resources. The proposed resource management framework is formulated on the basis of fluctuation in probability of resource utilization with respect to customers resource usage and allocates resources predicting the user behavior. Hermes, a framework that bridges the fog and cloud support analysis through federated query evaluations, is proposed in [50]. By selectively sampling the data generated by continuously sensing the environment, the framework reduces communication and memory consumption on fog nodes and cloud. Accrediting the users to share their resource in hosting applications will add dynamic to fog business models. The proposed user-in-the-loop approach by the authors in [51] can be used where incentives like reward programs, progressive tariffs or environmental indicators can be offered to users.

\section{Mobile Edge Computing}

MEC is a similar technology to fog computing. MEC brings the computational capacity within the Radio Access Network (RAN) to minimize delay and increase context awareness [52. But there are some basic differences with fog computing. For example, the locationof the fog nodes can vary between the edge devices and the cloud with a proximity of one or more hop. In MEC, nodes are located in the macro Base Station (BS) with the proximity of a single hop only. The access mechanism between fog node and edge device is heterogeneous (Bluetooth, Wi-Fi or mobile networks) but the access mechanism for MEC is only mobile networks. Although the internode connection is possible in both technologies, unlike the nodes in MEC, fog nodes are able to do internode communication even without a direct connection between them with the help of the cloud.

\subsection{Network Planning Process}

Planning a network is a very complex task due to many factors such as the network size, the heterogeneous nature of the equipment, the dynamic behavior of the users, 
the rapid changes in technology, etc. As a result, it is important to follow an organized methodolgy in planning and designing a network. The steps involved in planning and designing a network can be summarized as follows [53].

- Define design inputs: The important first step for network planning is to collect network input information and conduct requirement analysis. This step can be done by gathering comprehensive information on edge device traffic patterns and volume, estimated hardware type and cost, network capacity and QoS requirements. In general, the required data collection is complex and time consuming. Due to the unavailability of data, most of the time, the desired input is generated and considerable effort is put to validate the inputs with respect to real-time scenario.

- Network design: In this step, different design and dimensioning techniques are examined to create an appropriate algorithm for the network topology. This step includes recognizing the different network entities, geographical location, sizing and costing. Depending on the requirements, the output of the design can be the network topology, QoS, cost and so on.

- Optimization: The optimization of the network is done based on certain design precedence like delay, traffic, cost, power consumption, etc. The optimal solution is used as a benchmark and further improvement can be done. This step can be repeated with the evolution of network with different inputs and constraints.

- Performance analysis: After a number of iterations, a set of results can be obtained. This step requires the analysis and validation of the obtained results. The validation includes the sensitivity and statistical analysis of the uncertain variables.

There is lot of research focusing on the planning and design of networks in the field of computer and communication networks. For example, the authors in [54 [55] address the cellular network planning problem using appropriate optimization techiniques. The UMTS network planning problem is addressed in articles [56,57. The authors in [58,59] conducted a comprehensive research on the controller planning problem in SDN. Different planning tools for wireless networks as listed in $[60$ are developed by academics and industry researchers. The authors in [61] identify the Gigabit Passive 
Optical Network (GPON) planning problem and proposes a Lagrangian heuristic algoruthm to find the optimal placement of distribution points. Recently, the network function virtualization placement problem is explored by many researchers. The authors in 62 65 propose different placement models and algorithms with various optimization criteria.

As can be seen from the above paragraph, network planning is a huge field within the field of Operation Research (OR). In this thesis, we focus on the planning and design of the emerging concept of fog networks.

\subsection{Chapter Summary}

The literature review shows the importance of fog computing especially in the context of IoT. Fog computing can be seen as a complement to cloud computing resulting in a better overall system for the end users. As an infant technology, fog computing has many challenges that still need to be addressed before its deployment. Most importantly, network planning mechanisms should be adopted which satisfies the end users as well as the requirements of the service providers hosting the fog nodes.

At the best of our review, there is no work on the planning and design of fog networks. In fact, most papers dealing with fog computing already assume that fog nodes are already deployed and available to use. As a result, the next chapter introduces a new mathematical model for the planning and design of fog networks. 


\section{Chapter 3}

\section{Mathematical Model for Fog Planning Problem}

On a network of IoT devices with the coexistence of applications and heterogeneous services, it is not easy to find the best placement location for the installation of fog nodes. The planning problem can only be solved by the use of combinatorial methods and as the problem size gets larger, the number of possible combinations increases exponentially. For example, a network that has 5 possible locations and 200 edge device clusters, there are approximately $5^{200}$ different possible solutions for the planning problem. Given the limited capacity of fog nodes, assigning an edge device to a fog node for an optimal network is also a complex assignment problem. Furthermore, with the random distribution of edge device locations and their various requirements, it is challenging to determine the capacity of each fog node.

In this chapter, a mathematical model is formulated for the planning problem with a comprehensive explanation of the model. Given a set of edge device requests, the model simultaneously determines the optimal location, the number, and the capacity of the fog node(s) as well as the connection between the fog node(s) and the cloud. As the model has two different objectives (minimize delay and bandwidth), the Pareto optimality needs to be established, i.e., the delay in the network should not be worse off to minimize the traffic in the cloud.

The rest of this chapter is organized as follows. In the next section, we present the model formulation. Then, different techniques are described in order to solve optimization problems with multiple objectives functions. 


\subsection{Model Formulation}

The translation process from analyzed information into mathematical representation is known as model formulation. The model can be formulated by defining the objective function, and the set of constraints and specifying the input to the problem that defines the search space. The objective and each constraint is expressed algebraically in terms of the decision variables. The objective is to minimize network delay and cloud traffic with respect to each constraint.

To model edge devices, we use the notion of clusters where each cluster represents an agglomeration of edge device requests. We are using this approach to reduce complexity as typically, several users (hundreds or even thousands) are using the cloud at the same time in a given area.

\subsubsection{Assumptions}

To formulate the mathematical model, we assume the following information is known:

- There is no existing infrastructure meaning that we do the planning from a green field scenario.

- The location of all edge devices and the possible locations of each fog node (i.e., $x$ and $y$ coordinates). For each edge device, the amount of generated traffic and the link speed are also known.

- The bandwidth availability and the cost of each link type for the connection between the fog nodes and the cloud.

- The characteristics (i.e., memory, vCPU, cost) of different types of fog nodes that may be installed in a network.

- The location of the cloud. We assume that the cloud is located in a remote location and if an edge device cannot be served by the fog, it will be connected to the cloud. We also assume that the cloud has unlimited memory and vCPU.

- The fog nodes send a fraction of its total traffic to the cloud. This assumption is based on the fact that if an edge device is served by a fog node, most of the work will be done at the fog node level. However, some data may still be sent 
from the fog to the cloud for various reasons such as backup, enhanced services, etc.

The output of the planning problem focuses on the following:

- Selecting the optimal geographic location(s) to deploy fog node(s) in the network (i.e. where to deploy the fog nodes).

- Defining the optimal number of fog nodes that needs to be installed in the network.

- Specifying the type (capacity) of fog nodes to be installed in the network.

- Specifying the types of links used to interconnect the various network elements.

The primary goal of the Fog Planning Problem, denoted (FPP), is to find the best locations for installing fog nodes so that the network delay experienced by the users and the amount of traffic going towards the cloud are minimized.

\subsubsection{Sets}

- $U$, set of edge device clusters that are present in the network, $U=\left\{u_{1}, u_{2}, \ldots\right\}$

$-\lambda^{u}$, the total amount of memory required by a cluster of edge devices of type $u \in U$

$-\alpha^{u}$, the total number of $\mathrm{vCPU}$ required by a cluster of edge devices of type $u \in U$

- $\theta^{u}$, the number of packets requested to the fog nodes or the cloud by a cluster of edge devices of type $u \in U$

$-\kappa^{u}$, the link speed of a cluster of edge devices of type $u \in U$

- $N$, set of fog types that can be installed, $N=\left\{n_{1}, n_{2}, \ldots\right\}$

- $\lambda^{n}$, the total amount of memory available for a fog of type $n \in N$

- $\alpha^{n}$ the number of vCPU available for a fog of type $n \in N$

- $\theta^{n}$, the network interface capacity of a fog of type $n \in N$

$-\beta^{n}$, the number of nodes available for a fog of type $n \in N$ 
- $\phi^{n}$, the cost for a fog of type $n \in N$

- $L$, set of possible link types that can be used to interconnect fog nodes and cloud, $L=\left\{l_{1}, l_{2}, \ldots\right\}$

- $\omega^{l}$, the bandwidth (in Gbps) of a link of type $l \in L$

$-\xi^{l}$, the price (in dollars per meter) of a link of type $l \in L$

- $P$, set of possible locations to install the fog nodes, $P=\left\{p_{1}, p_{2}, \ldots\right\}$

\subsubsection{Constants}

- $\sigma$, average packet size (in bytes) sent by the edge devices.

- $\tau$, maximum budget (in dollars) allowed for the deployment of the fog.

- $t$, speed of light $\left(3^{*} 10^{8}\right.$ meters per second $)$.

- $h$, average number of hops packets take from edge devices to fog nodes.

- $k$, average processing delay (in seconds) per hop.

- $r$, percentage of traffic going to the cloud from fog nodes.

\subsubsection{Functions}

- Distance $(a, b)$ is a function that calculates the distance between points $a$ and $b$. The value of points $a$ and $b$ are the $x, y$ coordinates.

- $\psi$, transmission delay. It is a function that calculates the transmission delay (see Section 3.1.8).

- $\mu$, propagation delay. It is a function that calculates the propagation delay (see Section 3.1.8.

- $\gamma$, processing delay. It is a function that calculates the processing delay (see Section 3.1.8.

- $\mathrm{f}(a, b)$ is a function that takes the input parameter $a$ as the number of packets per second, $b$ the packet length in bytes and converts to bytes per second.

- $\mathrm{g}(a)$ is a function that converts a value from Mbps to bytes per second. 


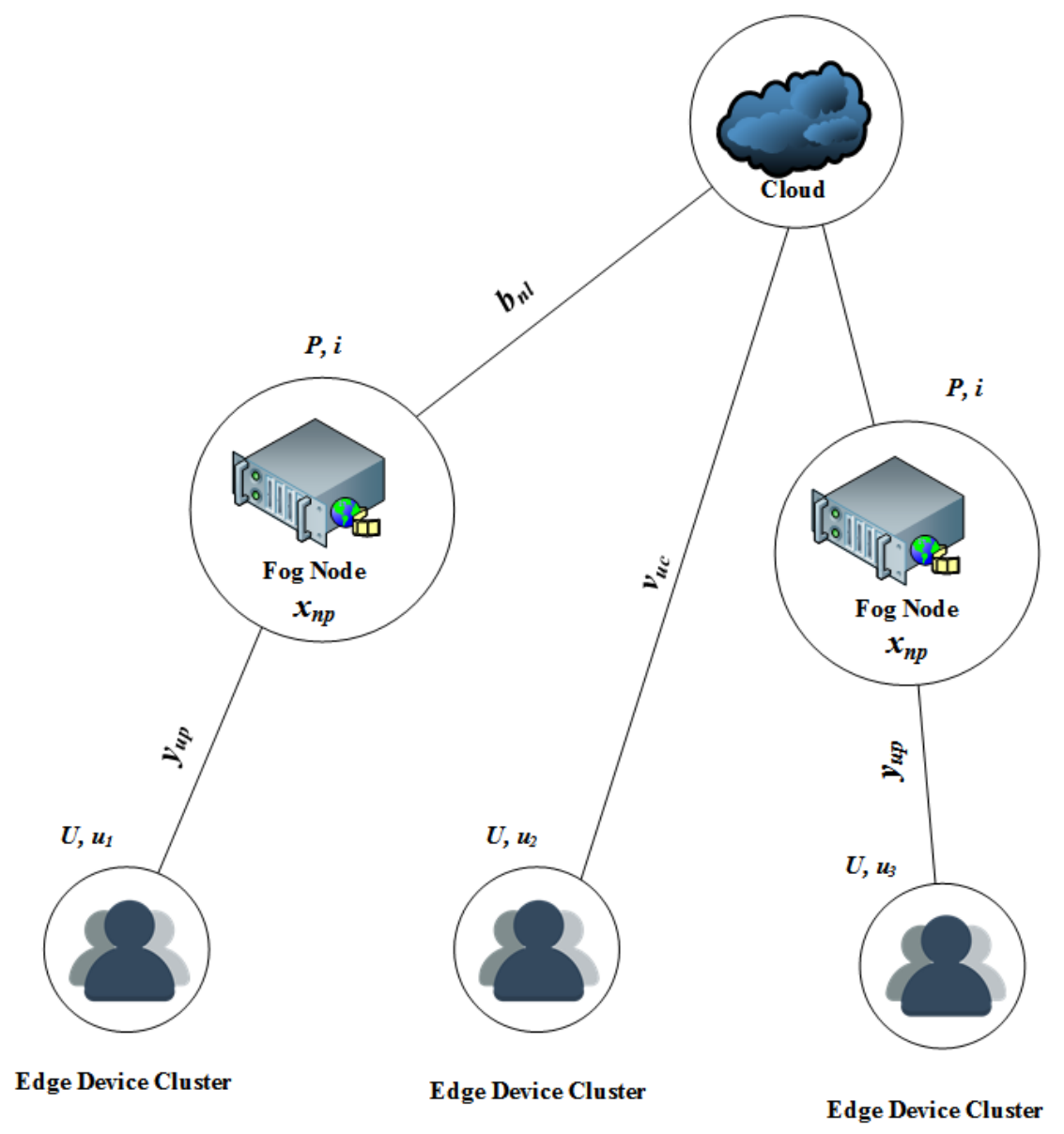

Figure 3.1: Graphical representation of the notations

\subsubsection{Decision Variables}

- $x_{n p}$, a 0-1 variable such that $x_{n p}=1$ if and only if the fog node $n \in N$ is installed at location $p \in P$;

- $y_{u p}$, a 0-1 variable such that $y_{u p}=1$ if and only if the edge device cluster $u \in U$ is connected to a location $p \in P$;

- $v_{u c}$, a $0-1$ variable such that $v_{u c}=1$ if and only if the edge device cluster $u \in U$ is connected to the cloud;

- $b_{p l}$, a $0-1$ variable such that $b_{p l}=1$ if and only if the fog node installed at location $p \in P$ is connected to the cloud with a link of type $l \in L$; 
Figure 3.1 is a graphical representation of the network showing the notations described earlier. The figure illustrates a three-tier "edge device-fog-cloud" architecture, with links between each network elements. It can be seen that, depending upon the load (and/or the type of application/service), an edge device cluster can either be connected to a fog node or to the cloud. Moreover, when a fog node is installed at a possible location, it must be connected to the cloud.

\subsubsection{Objective Function}

As shown in Equation 3.1, the objective function of the FPP, is to minimize the total delay of the network and the amount of traffic sent to the cloud.

$$
\text { Minimize }\left(D_{\text {total }}, \text { Traffic }\right)
$$

As shown in equations 3.2 to 3.5 , the total delay $\left(D_{\text {total }}\right)$ is the sum of the transmission delay $\left(D_{t}\right)$, propagation delay $\left(D_{n}\right)$ and processing delay $\left(D_{p}\right)$ in the whole network. On the other side, Equation 3.6 represents the total traffic going to the cloud which is the sum of the traffic from all edge devices connected to the cloud plus the traffic coming from the various fog nodes.

$$
\begin{gathered}
D_{\text {total }}=D_{t}+D_{n}+D_{p} \\
D_{t}=\sum_{u \in U} \sum_{p \in P} y_{u p} \psi+\sum_{u \in U} v_{u c} \psi \\
D_{n}=\sum_{u \in U} \sum_{p \in P} y_{u p} \mu+\sum_{u \in U} v_{u c} \mu \\
D_{p}=\sum_{u \in U} \sum_{p \in P} y_{u p} \gamma+\sum_{u \in U} v_{u c} \gamma \\
\text { Traffic }=\sum_{u \in U} \sum_{l \in L} v_{u c} \theta^{u}+\sum_{p \in P} \sum_{l \in L} b_{p l} \theta^{u} r
\end{gathered}
$$

\subsubsection{Formulation of the Constraints}

The model aims to minimize the network delay as well as the traffic entering the cloud. The set of constraints explained below restricts the minimization function. 
- Uniqueness constraints: Uniqueness constraints enforce that at most one fog node is installed at a given location. In other words, we cannot install two or more fog nodes at the same location.

$$
\sum_{n \in N} x_{n p} \leq 1 \quad(p \in P)
$$

- Edge device assignment constraints: The assignment constraints are used to make sure that each device is assigned to exactly one location (i.e., the fog or the cloud).

$$
\sum_{p \in P} y_{u p}+v_{u c}=1 \quad(u \in U)
$$

- Node assignment constraints: The assignment constraints enforce that each installed fog node is connected to cloud.

$$
\sum_{n \in N} x_{n p}=\sum_{l \in L} b_{p l} \quad(p \in P)
$$

- Node capacity (vCPU) constraints: The capacity constraints for vCPU at the node level make sure that the number of $\mathrm{vCPU}$ required by the edge devices does not exceed the capacity of the fog node.

$$
\sum_{u \in U} y_{u p} a^{u} \leq \sum_{n \in N} x_{n p} a^{n} \quad(p \in P)
$$

- Node capacity (memory) constraints: The capacity constraints for memory at the node level make sure that the total amount of memory required by the edge devices does not exceed the capacity of the fog node.

$$
\sum_{u \in U} y_{u p} \lambda^{u} \leq \sum_{n \in N} x_{n p} \lambda^{n} \quad(p \in P)
$$

- Network Interface Capacity (NIC) constraints: The capacity constraints at the node interface level ensure that the total inbound bandwidth required by the edge devices does not exceed the fog node capacity. 


$$
\sum_{u \in U} y_{u p} f\left(\theta^{u}, \sigma\right) \leq \sum_{n \in N} x_{n p} g\left(\theta^{n}\right) \quad(p \in P)
$$

- Link capacity constraints: The capacity constraints at the cloud interface level ensure that the total inbound bandwidth from the fog nodes to the cloud does not exceed the link capacity.

$$
\sum_{u \in U} y_{u p} f\left(\theta^{u}, \sigma\right) r \leq \sum_{l \in L} b_{p l} g\left(\omega^{l}\right) \quad(p \in P)
$$

- Inventory constraints: Inventory capacity constraints of the fog nodes make sure that we do not use more than what we have in inventory for a fog type.

$$
\sum_{p \in P} x_{n p} \leq \beta^{n} \quad(n \in N)
$$

- Budget constraint: The budget constraint makes sure that the total cost of the network does not exceed the maximum allowed budget.

$$
\sum_{n \in N} x_{n p} \phi^{n}+\sum_{l \in L} b_{p l} \operatorname{dist}(p, c) \xi^{l} \leq \tau \quad(p \in P)
$$

- Integrality constraints: States that the domain of the following decision variables is a set of binary numbers.

$$
\begin{array}{ll}
x_{n p} \in B & (n \in N, p \in P) \\
y_{u p} \in B & (u \in U, p \in P) \\
v_{u c} \in B & (u \in U, l \in L) \\
b_{p l} \in B & (p \in P, l \in L)
\end{array}
$$

\subsubsection{Delay Computation}

The latencies for the network are computed using the transmission, propagation and processing delays. It would also be interesting to consider the queuing delay but 
as this keeps changing over time, a static model like the one presented in this chapter does not allow to include it.

The transmission delay is the time taken for a process to send the information to the wire. This depends on the link speed that is used and the packet size that is to be sent to the edge device from the fog or cloud. The formula to calculate the transmission delay is shown below where $\sigma$ is the amount of data (bytes) and $w^{l}$ represents the link speed (bytes/sec).

$$
\text { Transmission delay }(\psi)=\frac{\sigma}{w^{l}}
$$

The propagation delay is the time taken to transmit a signal from a source to a destination. This depends on the medium used and the distance between the source and the destination. The propagation delay is calculated as distance divided by the speed at which the signal propagates in the medium. Depending on the medium that is used, the propagation speed varies. The propagation speed of wireless communications is the speed of light. For copper wires, the speed varies from $0.59 t$ to $0.77 t$ [66]. In the proposed model, we use $0.59 t$ for the speed of copper wire which can be also be used in premises where fiber or other medium cannot be deployed [67].

$$
\text { Propagation delay }(\mu)=\frac{\operatorname{Distance}(a, b)}{0.59 * t}
$$

Each router or switch in the data path adds a finite amount of delay as the packet is received, processed, and then forwarded. This includes the time taken at each layer of the TCP/IP down until the bit level layer. Using features that are supported with hardware assistance can greatly reduce the processing delay. Typically, the processing delay with a hardware-assisted switch will be in the 4 -to-20 microseconds range 68. In the worst case scenario, even when using a software assistance, the most reasonable processing delay that can be expected in practice should be 25 microseconds per hop $(h)$, which has been used in our calculation.

$$
\text { Processing delay }(\gamma)=k * h
$$




\subsection{Model with Pareto Optimality}

Since the formulation of the problem is a multiobjective optimization model, we need to simultaneously minimize the two objective functions expressed in equations 3.2 and 3.6. Sometimes, the minimum network delay will increase the traffic in the cloud and vice versa. Therefore, to ensure the fairness of the network delay and the traffic in the cloud, we need to find the Pareto optimal solution. In this section, we present three different methods that we used to find solutions to our problem.

\subsubsection{Weighted Sum Optimization}

The weighted sum strategy converts the multiobjective problem of optimizing the main objective function into a scalar problem by building a weighted sum of all the objectives [69]. Therefore, the proposed objective function can be rewritten as:

$$
\text { Minimize }\left(w_{1} D_{\text {total }}^{\text {norm }}+w_{2} \text { Traffic }{ }^{\text {norm }}\right)
$$

where $w_{1}$ and $w_{2}$ are weighted coefficient and $D_{\text {total }}^{\text {norm }}$ and Traffic $c^{\text {norm }}$ are the normalized objective functions as they have different scales. Equations 3.24 and 3.25 are used to normalize the objective functions.

$$
\begin{gathered}
D_{\text {total }}^{\text {norm }}=\frac{D_{\text {total }}^{\text {max }}-D_{\text {total }}}{D_{\text {total }}^{\text {max }}-D_{\text {total }}^{\text {min }}} \\
\text { Traffic }^{\text {norm }}=\frac{\text { Traffic }}{\text { Trax }- \text { Traffic }} \text { Trax }^{\text {Traffic }} \text { Trin }^{\text {max }}
\end{gathered}
$$

The weighted coefficients define the contribution of each objective function in the modified model. Even with a proper knowledge of the problem, it is not easy to specifically select the weight of the coefficients in the modified objective function. Therefore, an additional constraint 3.26 is added which states that the sum of the weighted coefficients $w_{1}$ and $w_{2}$ should be 1 .

$$
w_{1}+w_{2}=1
$$

During simulations, both weighted coefficients are varied in steps of 0.1 with respect to the added constraint to obtain a Pareto front. 


\subsubsection{Hierarchical Optimization}

Hierarchical optimization is generally solved by the sequential optimization method. Here, the objective functions are ranked in order of importance. From the literature review, it can be assumed that the fog nodes will be used extensively for latency sensitive applications [31,33,35]. Hence, the model is solved using Traffic as the lone objective function with an additional constraint that do not allow the value of the delay function to exceed a set of prescribed fractions of its optimal value [70]. In other words, we try to improve the less important criteria through minimal loss of the most important criterion.

$$
\text { Minimize } D_{\text {total }}
$$

In the first step, we solve Equation 3.27 with respect to constraints 3.7 3.19 . In the second step, we solve Equation 3.28 with the additional constraint 3.29 .

$$
\begin{gathered}
\text { Minimize Traffic } \\
D_{\text {total }} \leq D_{\text {total }}^{\text {min }} * \delta
\end{gathered}
$$

where $\delta$ is the added value for delay. We vary the $\delta$ with a set of values to obtain a Pareto optimal front.

\subsubsection{Trade-Off Method}

This method involves optimizing a primary objective, and expressing the other objectives in the form of inequality constraints [71]. In our proposed trade-off model, we consider $D_{\text {total }}$ as the primary objective function due to the reason explained before and Traffic as an inequality constraint with specified values of traffic in the cloud. We solve the objective function show in Equation 3.27 subject to:

$$
\text { Traffic } \leq \epsilon
$$

and all other constraints $3.7-3.19$. However, it is not easy to find the precise $\epsilon$ value for the optimal solution. For this, the range of Traffic $c^{\text {max }}$ and Traffic ${ }^{\text {min }}$ is divided into ten equal intervals and we use the eleven values as the varying $\epsilon$ value to obtain an optimal Pareto set curve 72$]$. 


\subsection{Determining the Best Compromise Solution}

From each method of the multiobjective model, we obtain a set of non-dominated solutions forming a Pareto front. Since there can be an uncertainty in decision makers preference, it can be assumed there is a fuzziness in the goal of each objective function. The membership functions define this fuzziness by representing the degree of fuzziness in some fuzzy sets using values in the range $[0,1]$.

In this thesis, we adapt a fuzzy-based mechanism [73] which is used to find out a compromised solution on the Pareto front of each problem. The fuzzy mechanism looks at the way the solutions are contributing to each objective and assigns a fuzzy variable. When the solutions in a Pareto front are very close together, the mechanism shows a possible way of finding the best compromise solution. In this mechanism, a membership value for the $i^{t h}$ objective of the $j^{\text {th }}$ solution in the Pareto front is calculated using the membership function as:

$$
\mu_{i}^{j}= \begin{cases}1 & \text { if } F_{i} \leq F_{i}^{\text {min }} \\ \frac{F_{i}^{\text {max }}-F_{i}}{F_{i}^{\text {max }}-F_{i}^{\text {min }}} & \text { if } F_{i}^{\text {min }}<F_{i}<F_{i}^{\text {max }} \\ 0 & \text { if } F_{i} \geq F_{i}^{\text {max }}\end{cases}
$$

$\mu_{i}^{j}$ indicates how well the $j^{\text {th }}$ solution is able to satisfy the $i^{\text {th }}$ objective in a Pareto optimal set. The sum of the membership value of all objectives of the $j^{\text {th }}$ solution suggests how well it satisfies all the objectives. Given $N$ solutions in a Pareto front and $M$ objective functions for each solution, the achievement of each solution with respect to all the $N$ solutions can be calculated by:

$$
\mu^{j}=\frac{\sum_{i=1}^{M} \mu_{i}^{j}}{\sum_{j=1}^{N} \sum_{i=1}^{M} \mu_{i}^{j}}
$$

The solution with a maximum value of $\mu^{j}$ is a compromise solution that can be accepted by the decision maker. 


\subsection{The Hypervolume Indicator}

The Hypervolume (HV) indicator was first proposed in [74]. In recent years, the hypervolume indicator has been widely used in multiobjective optimization to evaluate various search algorithms.

If an objective space contains solution that are considered as points, the ndimensional space that is contained by a solution set is called hypervolume. In other words, the hypervolume is the n-dimensional volume of the set relative to some reference point. The reference point usually refers to the worst possible point in the solution set. The hypervolume of a set is the total dominated space of the solutions in the set. The single unary value of HV gives a measure of the spread of the solutions along the Pareto front. A set with large HV is always desirable as it presents a better set of trade-offs as it covers a larger area in the solution space.

Given a non-dominated set of solutions $S$, for each solution $i \in S$ there is a hypercube $v_{i}$ with a reference point $r$ where the solution $i$ is the diagonal corners of the hypercube. With the union of all the hypercubes, the hypervolume can be calculated by:

$$
H V=\cup_{i=1}^{|S|} v_{i}
$$

Figure 3.2 shows an example of $\mathrm{HV}$ for a 2-dimensional minimization problem with a set of non-dominated solution $\mathrm{S}=\left\{a_{1}, a_{2}, a_{3}, a_{4}\right\}$ and reference point $r$. As we can see from the figure, the yellow area is the hypervolume contributed by the four non-dominated solutions with respect to the reference point.

\subsection{Chapter Summary}

In this chapter, we formulated an exact mathematical model for the planning and design of fog networks. The objective of the model is twofold: 1) minimize the delay experienced by the users and 2) minimize the amount of traffic that must go to the cloud, subject to a set of realistic constraints. Since we formulated the problem as a multiobjective mathematical model, we reviewed three different methods and modified our model with additional constraints to obtain Pareto optimal solutions. Finally, two different methods and functions fuzzy-based mechanism and HV indicator were 
CHAPTER 3. MATHEMATICAL MODEL FOR FOG PLANNING PROBLEM 39

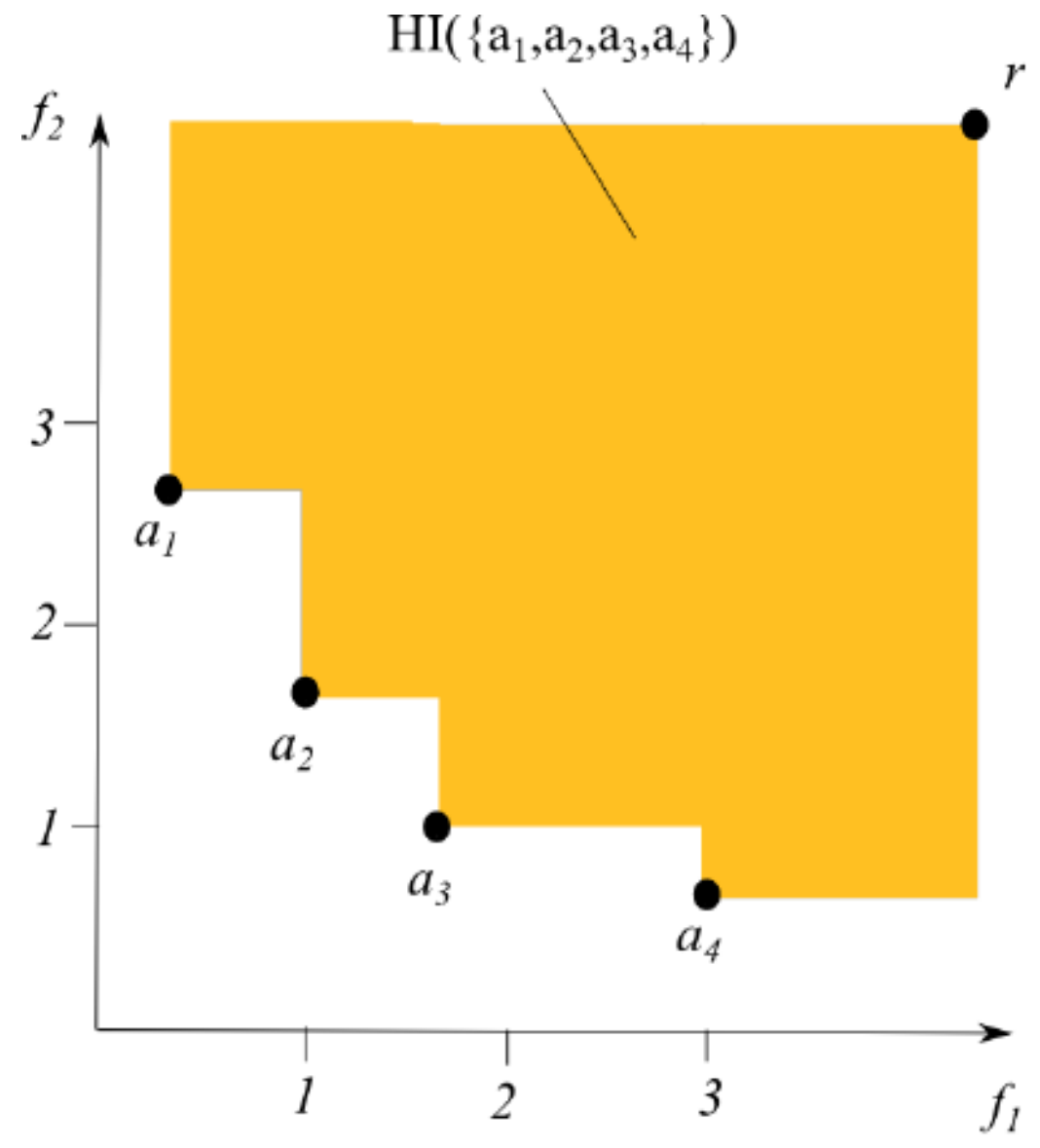

Figure 3.2: HV enclosed by non-dominated solution $a_{1}, a_{2}, a_{3}$ and $a_{4} \quad 75$

introduced and explained in the last two sections of the chapter. The aforementioned methods are used to analyze and better understand the results. In the next chapter, we analyze the results and performance of the proposed methods with different inputs. 


\section{Chapter 4}

\section{Results and Analysis}

In this chapter, we solve the planning problem modeled in Chapter 3 with different input. Then we do a complete analysis of the obtained results to better understand the performance of the planning model. More precisely, the chapter begins with a summary of the steps involved when solving the FPP. In the next section, we solve a detailed example to understand how each of the constraints work in the model. Then, we analyze the results from small scale to large scale problems followed by a study of the impact of increasing the number of possible placement locations. Finally, we summarize the chapter in the last section.

\subsection{Framework for the Fog Planning Problem}

There are many steps that need to be taken to find the optimal solution for FPP. Figure 4.1 summarizes the steps that we used for each problem in our planning model. First, the input information is generated and the problem is solved with the planning model in Chapter 3. The problem is solved for both single and multiobjective functions. For the multiobjective optimization, three different methods are used to generate a Pareto front. Next, the fuzzy-based mechanism described in Section 3.3 is used in determining the best compromise result from the Pareto set. The hypervolume of the Pareto front is calculated using Equation 3.33. Finally, the results obtained from all the steps are analyzed for better understanding of the FPP.

Figure 4.2 shows the flowchart for solving the FPP using the weighted sum method. In the first step, we generate the input for the planning problem. Initially, the value for $w_{1}$ is set to 1 and $w_{2}$ is set to 0 . Then the modified model from Section 3.2 .1 is 


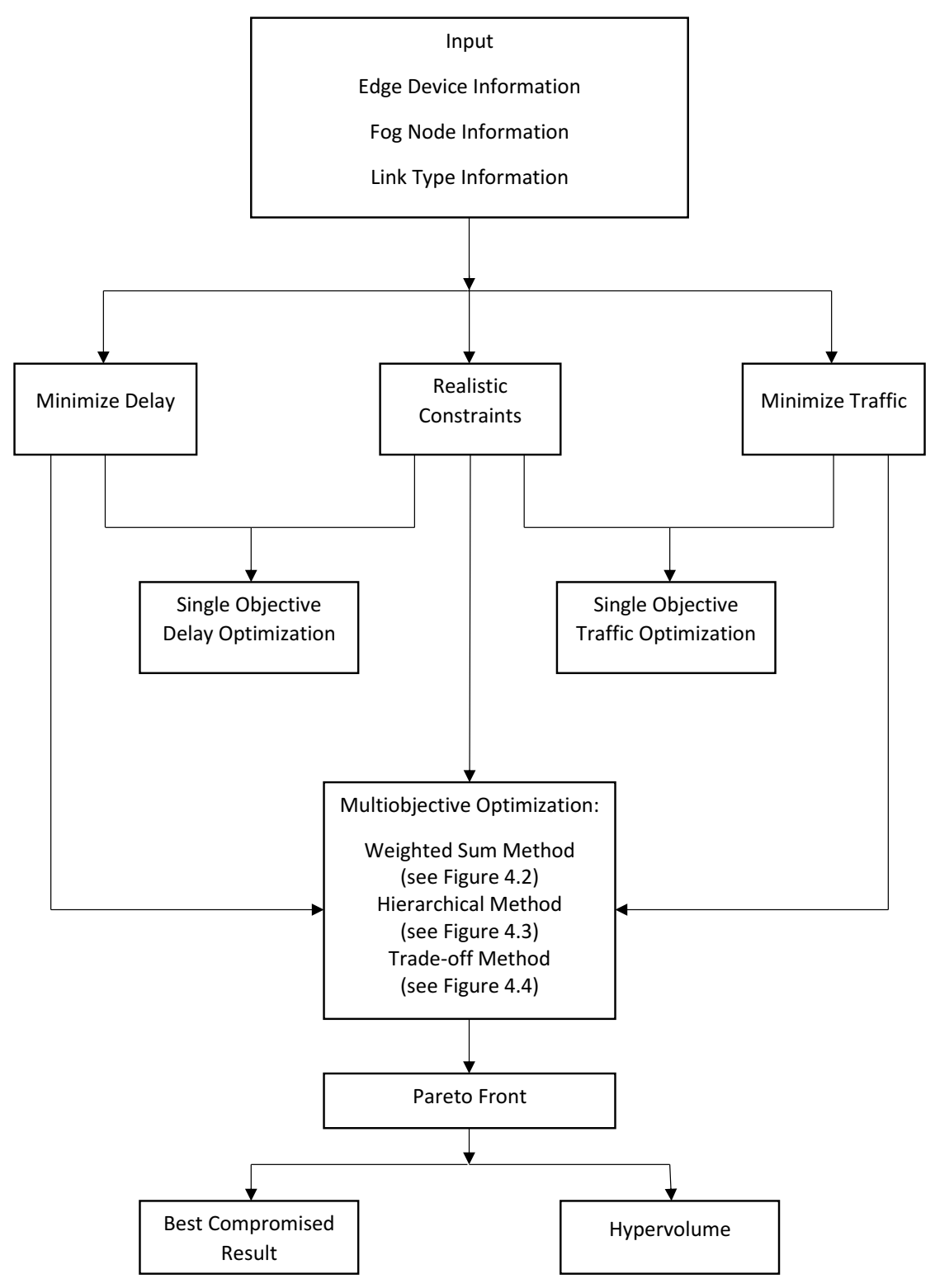

Figure 4.1: Steps to solve the FPP 


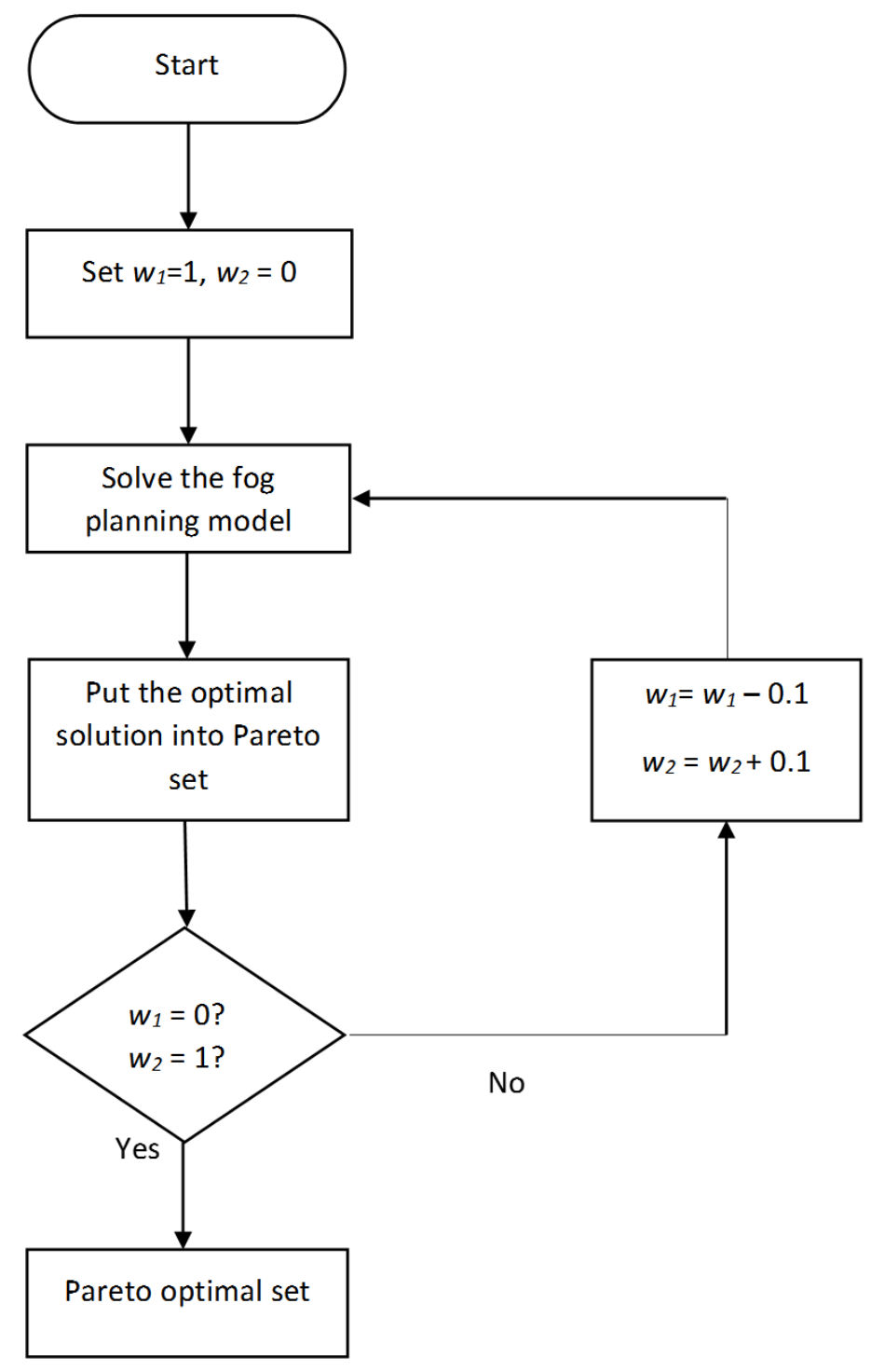

Figure 4.2: Steps to solve the FPP with the weighted sum method 
solved and the returned objective function values are stored in the Pareto set. Then the terminating condition is checked and if it is not satisfied, the model is solved one more time with updated weighted coefficient values and the new solution being stored in the Pareto set. This step is repeated until the terminating condition is satisfied. Once the terminating condition is satisfied, a Pareto optimal set is obtained from the stored objective function values. The fuzzy-based mechanism described in Section 3.3 is used to extract the best compromised result from the Pareto optimal set, which is used as the final result for analysis. The procedure outlined in the flowchart is used each time we solve a fog planning problem with the weighted sum method.

The steps for solving the hierarchical method are shown in Figure 4.3. Initially, the inputs for the FPP are randomly generated. In the next steps, the value of $\delta$ is set to 1.0001 and the modified hierarchical model presented in Section 3.2 .2 is solved. The solution results are stored in the Pareto optimal set. Next, the terminating condition is checked and if not satisfied, the $\delta$ value is updated with $\delta^{\natural}$, where $\delta^{\natural}$ equals to 0.0001 and the problem is solved one more time with the updated $\delta$ value and the results are stored in the Pareto set. This process is repeated until the terminating condition is satisfied, which is $\delta$ equal to 1.0008 . When the terminating condition is satisfied, a Pareto optmal set is obtained from all the solutions from Pareto set. Finally, the best compromised result is obtained from the Pareto optimal set using the fuzzy decision approach. This procedure is used when solving all the problem in FPP. 


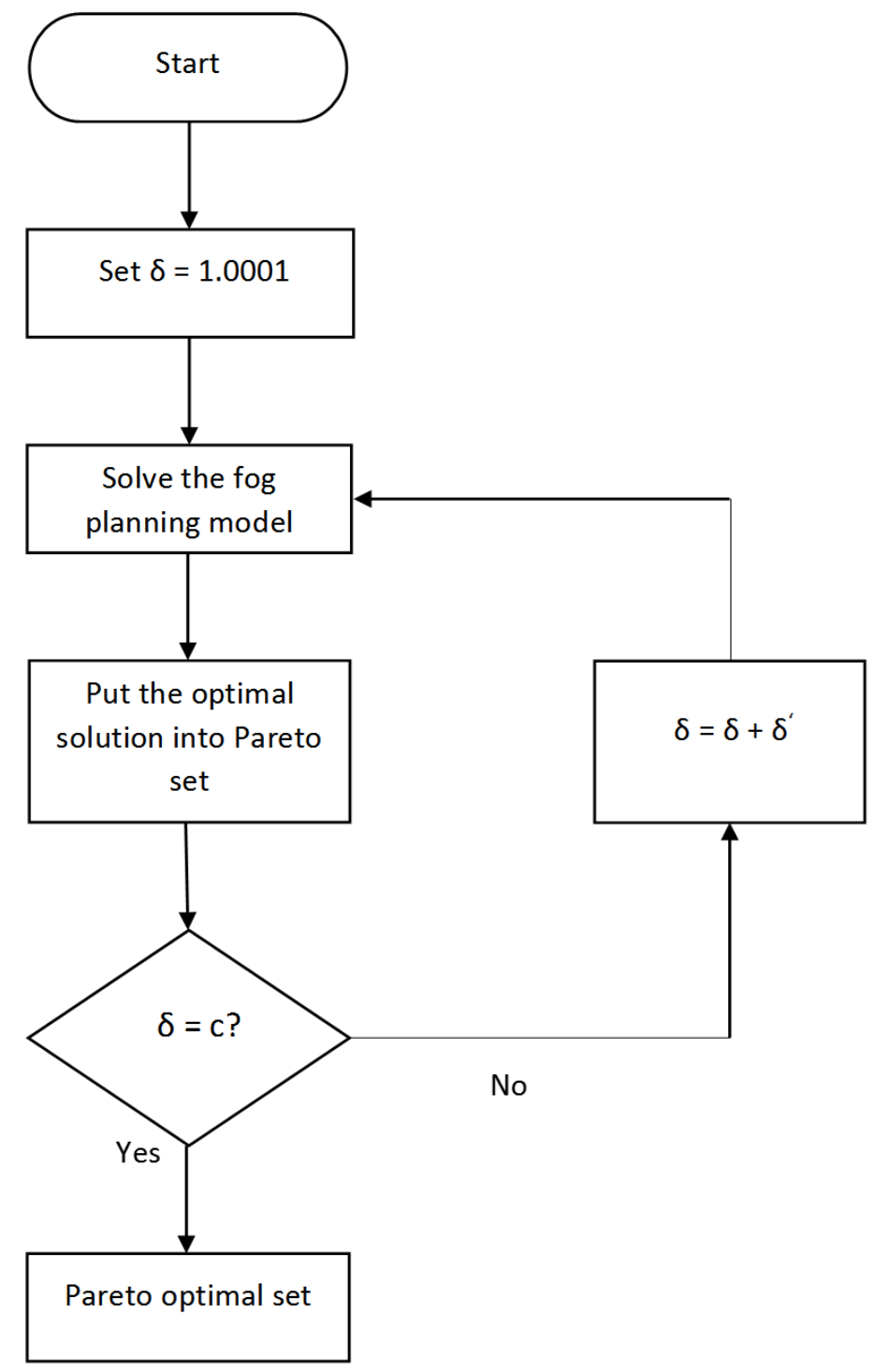

Figure 4.3: Steps to solve the FPP with the hierarchical method 


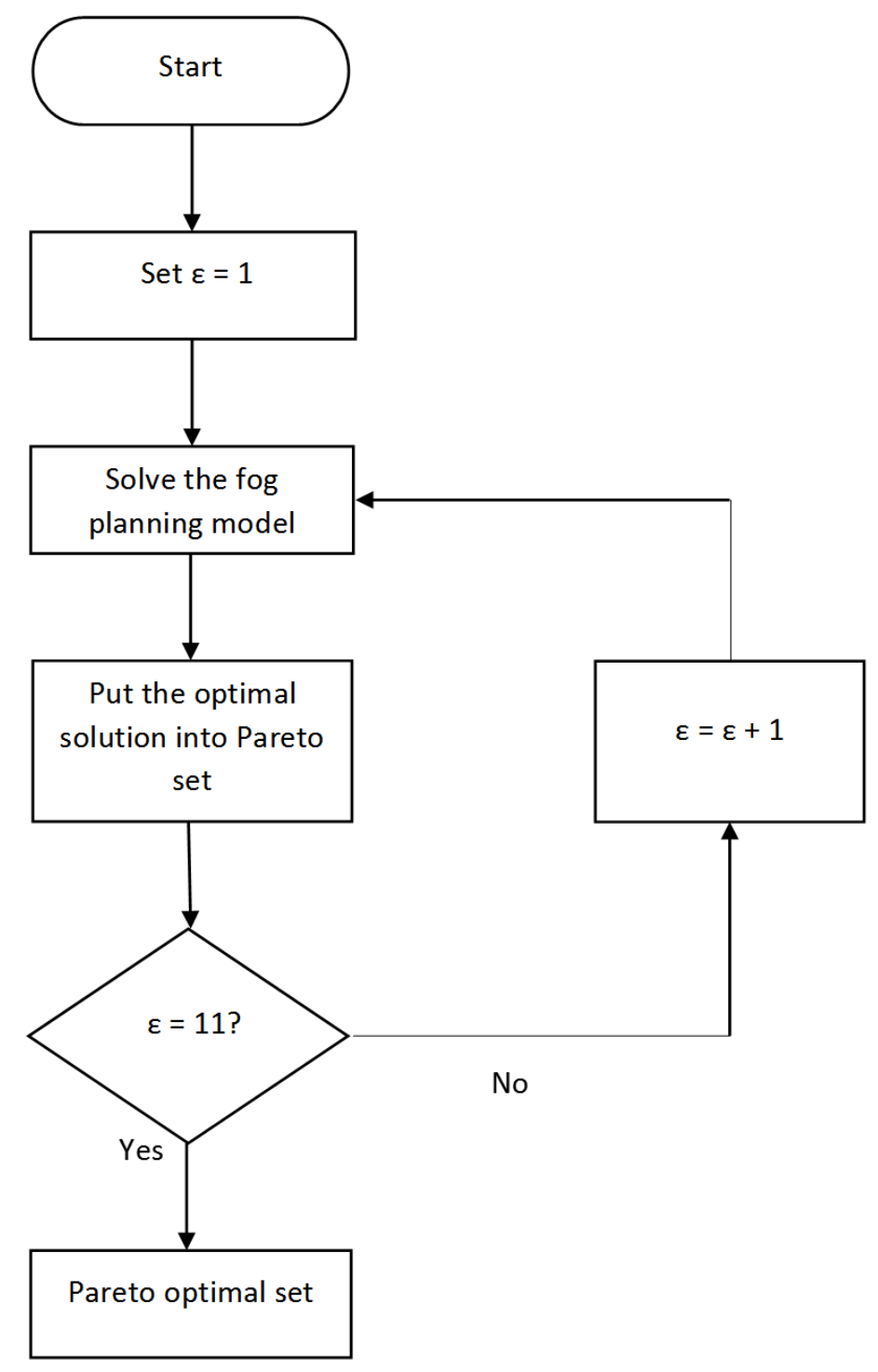

Figure 4.4: Steps to solve the FPP with the trade-off method

The flowchart to solve the FPP using the trade-off method is illustrated in Figure 4.4. The method uses the same randomly generated inputs used for the previous two multiobjective optimization methods. The $\epsilon$ value is set to 1 and the modified trade-off model in Section 3.2 .3 is executed. The optimal solutions from the model 
are stored in the Pareto optimal set. In the next step, the terminating condition is checked and if it is not satisfied, the problem is solved with a new $\epsilon$ value, which is a specified value of traffic in the cloud. The model is repeatedly solved until the terminating condition is satisfied. When the terminating condition is checked out, an optimal Pareto front is obtained. Finally, the fuzzy-based mechanism is used to obtain the best compromised result for the trade-off method.

\subsection{Detailed Example}

The simple example presented in this section is used to explain and show how the mathematical model works. In this example, we want to plan and design a brand new fog network in order to accommodate 10 edge device clusters by finding the optimal number and location for the fog node(s) while considering the nodes processing capacity, the bandwidth requirements of the edge device clusters and link and nodes inventory. Once the result is obtained, it will be analyzed and the final conclusion is made by showing the total network delay and traffic in the cloud with the optimal placement variables.

Figure 4.5 shows the area to be planed. As it can be seen, there are 10 edge device clusters that need to be connected to either a fog node or the cloud depending upon their request with 5 possible fog node placement locations. As we assume, that the cloud is located in a remote location, is not shown in the figure.

In this simple scenario, we have two types of fog nodes. The characteristics of both types are given in Table 4.1. Similarly, as shown in Table 4.2, there are two types of link with a bandwidth of 10 Mbps and 100 Mbps respectively. Table 4.3 shows the optimization input parameters with randomly generated edge device and fog node locations over an area of $100 \mathrm{~km}^{2}$. The size of each requested packet is 1250 bytes and there are only three fog nodes available (two of type a and one of type b). The parameters of each cluster are given as input to the model and are listed in Table 4.4. The first column shows the edge device cluster index. Columns 2 and 3 show the location of the edge device cluster in the planned area, whereas columns 3-6 show the profile of each edge device cluster which includes the requested memory and vCPU, 


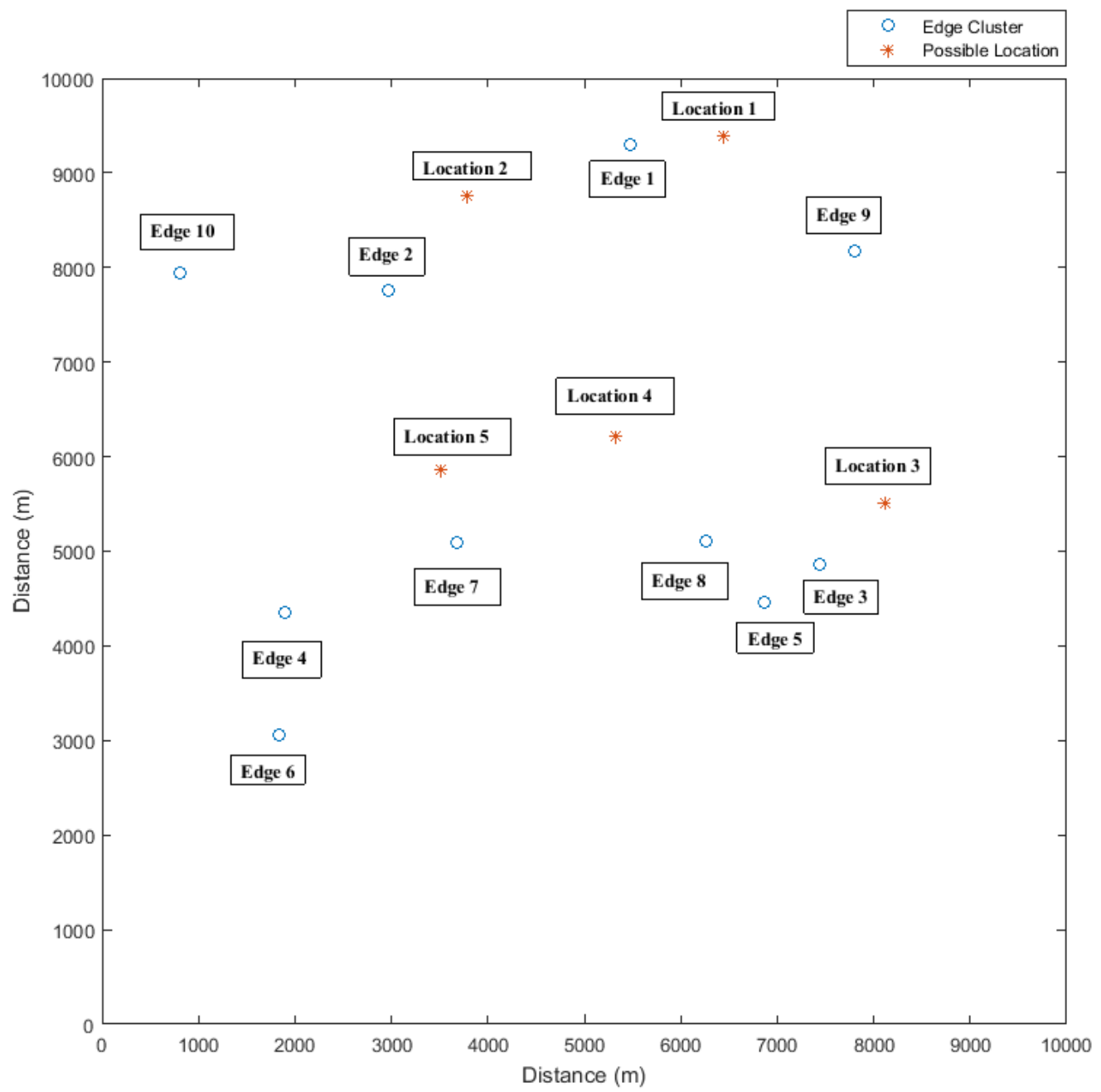

Figure 4.5: Edge device locations and potential locations of fog nodes

Table 4.1: Fog type features for the planning example

\begin{tabular}{|c|c|c|}
\hline Fog Types & a & b \\
\hline vCPU $\left(\alpha^{n}\right)$ & 5 & 10 \\
\hline Memory $\left(\lambda^{n}\right)$ & 15 & 30 \\
\hline NIC $\left(\theta^{n}\right)$ & 45 & 100 \\
\hline \# of Nodes $\left(\beta^{n}\right)$ & 2 & 1 \\
\hline $\operatorname{Cost~}\left(\phi^{n}\right)$ & 1000 & 2000 \\
\hline
\end{tabular}


Table 4.2: Link type features for the planning example

\begin{tabular}{|c|c|c|}
\hline Link Types & a & b \\
\hline Bandwidth $\left(\omega^{l}\right)$ & $10 \mathrm{Mbps}$ & $100 \mathrm{Mbps}$ \\
\hline Cost/metre $\left(\xi^{l}\right)$ & $\$ 0.05$ & $\$ 0.25$ \\
\hline
\end{tabular}

Table 4.3: Topology and optimization input parameters for the example

\begin{tabular}{|c|c|}
\hline Simulation area & $10 \times 10 \mathrm{~km}$ \\
\hline Number of edge device clusters & $10($ random $)$ \\
\hline Maximum Placements & 5 (random) \\
\hline Packet size $(\sigma)$ & 1250 Bytes \\
\hline
\end{tabular}

Table 4.4: Edge device input parameters for each cluster

\begin{tabular}{|c|c|c|c|c|c|c|}
\hline \multirow{2}{*}{ Edge device cluster index } & \multicolumn{2}{|c|}{ Location $(\mathrm{m})$} & \multicolumn{3}{|c|}{ Requested } & \multirow{2}{*}{ Network Access Bandwidth (Mbps) } \\
\hline & $x$ & $y$ & Memory & vCPU & Traffic (Mbps) & \\
\hline 1 & 5470 & 9294 & 6 & 2 & 12 & 10 \\
\hline 2 & 2964 & 7757 & 5 & 1 & 16 & 21 \\
\hline 3 & 7447 & 4868 & 9 & 4 & 13 & 14 \\
\hline 4 & 1890 & 4359 & 3 & 12 & 16 & 17 \\
\hline 5 & 6868 & 4468 & 10 & 3 & 33 & 28 \\
\hline 6 & 1836 & 3064 & 18 & 1 & 26 & 8 \\
\hline 7 & 3685 & 5085 & 6 & 3 & 41 & 11 \\
\hline 8 & 6257 & 5108 & 5 & 3 & 11 & 22 \\
\hline 9 & 7803 & 8176 & 35 & 2 & 23 & 14 \\
\hline 10 & 812 & 7948 & 13 & 8 & 44 & 9 \\
\hline
\end{tabular}


as well as the traffic and the network access bandwidth.

To solve the problem, we used CPLEX $12.7[76]$. All the parameters in the optimizer were set to default except that we set a Time Limit (TL) of 1.5 hours. This means that if the optimal solution is not found after 1.5 hours of computation, CPLEX will simply return the best solution obtained so far. We run the simulation on a PC with an Intel i5 processor running at $3.00 \mathrm{GHz}$ with a total memory of $12 \mathrm{~GB}$.

The goal of the model is to select the best locations to install the fog nodes such that all constraints are satisfied with minimum delay in the network and minimum amount of traffic going to the cloud. As it is a small problem, the solution search space is also small resulting in obtaining the same solution for every iterations for the three multiobjective methods. The optimal solution obtained from CPLEX has a total delay of 0.00702 second and traffic of 117.19 Mbps. From Figure 4.6, we can see that 6 edge device clusters are connected to fog nodes installed at 3 different locations. In the figure, the solid black lines show the connections between the edge device clusters and the fog nodes, the dash line shows the connection between the edge device clusters and the cloud and the dash-dot line presents the connection between the installed fog nodes and the cloud. The fog nodes that were not part of the optimal solutions (i.e. locations 1 and 4) were not connected as seen in the figure.

By visual observation from Figure 4.6, we can see that edge device cluster 3 is close to location 3 with 922 meters where a fog of type a is installed. Although edge device cluster 1 is close to location 1 (978 meters), installing a fog node there will be far for edge device cluster 2 increasing the overall network delay. So, a fog of type a is installed in location 2 connecting both edge device cluster 1 and 2. Placement location 5 is used to install a fog of type b as it is connected to three edge device clusters which are closer. All of the installed fog nodes are connected to the cloud which is approximately 90,000 meters away from each of the fog nodes.

\section{Capacity Constraints}

Constraints 3.103 .12 state that the capacity of a fog node should be greater or equal to the sum of all edge device requirements that are connected to it. For example, edge device cluster 9 is closer to location 1, but it requests $35 \mathrm{MB}$ of memory which is above the capacity of both fog types. Hence, it is connected to the cloud. The fog node installed at location 5 can accommodate edge device cluster 4 in terms of all 


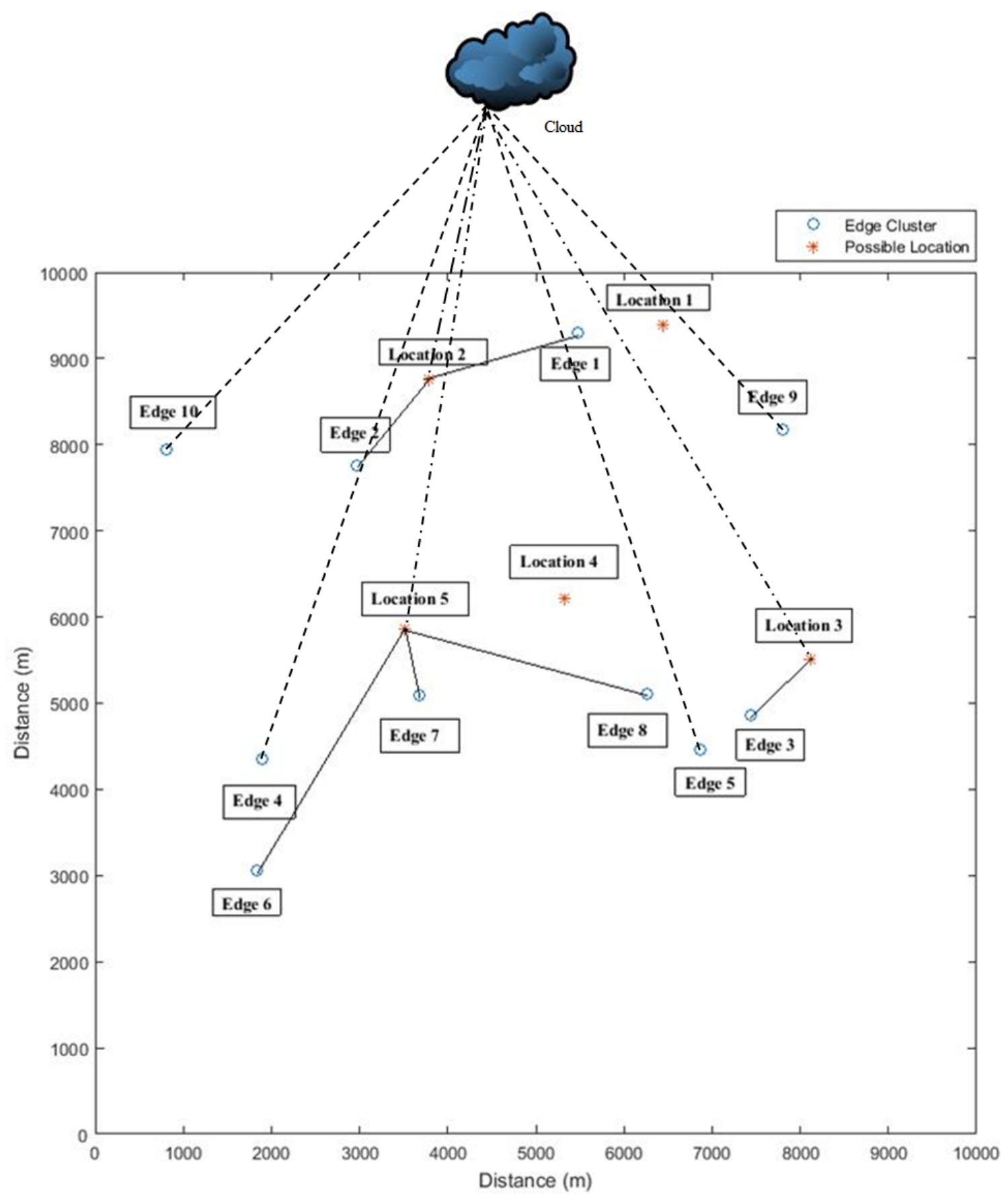

Figure 4.6: Optimal solution found by Cplex 
the constraints except Equation 3.10. Therefore, edge device cluster 4 is connected to the cloud.

\section{Inventory and Budget Constraints}

Equation 3.14 limits the number of each fog node type that can be installed. In the final solution, the solver will make sure that each fog node type is not used more than the maximum number of nodes available as indicated in the fog nodes input information in Table 4.1. In this case, two fog nodes of type a and one fog node of type $\mathrm{b}$ are installed in the network. Constraint 3.15 restricts the solver to go over budget. For an ideal situation, there can be a fog node installed in every location, but as we have restricted the budget to $40 \%$ of the maximum cost, a limited number of fog node can be installed.

\section{Link Bandwidth between Fog Nodes and Cloud}

Equation 3.9 ensures that there is a connection between all installed fog node and the cloud. Links from Table 4.2 are in Mbps and need to be converted to bytes per second. Function $g\left(\omega^{l}\right)$ converts between the two units for link type $(l \in L)$. In this simple example, we assume that only $1 \%$ of the traffic coming to a fog node is sent to the cloud for further processing. Since, we do not want to go over budget, the lowest link type is used.

\section{Optimal Solution}

In summary, the decision variables selected are:

- Fog node placement $\left(x_{n p}\right): x_{12}, x_{13}, x_{25}$

- Edge device and fog node connection $\left(y_{u p}\right): y_{12}, y_{22}, y_{33}, y_{65}, y_{75}, y_{85}$

- Edge device and cloud connection $\left(v_{u c}\right): v_{41}, v_{51}, v_{91}, v_{101}$

- Fog node and cloud connectivity $\left(b_{n l}\right): b_{21}, b_{31}, b_{51}$ 
Table 4.5: Delay formulation of the planning example

\begin{tabular}{|c|c|c|}
\hline Transmission Delay $(\psi)$ & Propagation Delay $(\mu)$ & Processing $(\gamma)$ \\
\hline $0.000125 y_{12}$ & $9.98192 \mathrm{E}-06 y_{12}$ & $0.000125 y_{12}$ \\
\hline $5.95238 \mathrm{E}-05 y_{22}$ & $7.32655 \mathrm{E}-06 y_{22}$ & $0.000125 y_{22}$ \\
\hline $8.92857 \mathrm{E}-05 y_{33}$ & $5.20565 \mathrm{E}-06 y_{33}$ & $0.000125 y_{33}$ \\
\hline $7.35294 \mathrm{E}-05 y_{65}$ & $1.84593 \mathrm{E}-05 y_{65}$ & $0.000125 y_{65}$ \\
\hline $4.46429 \mathrm{E}-05 y_{75}$ & $4.54915 \mathrm{E}-06 y_{75}$ & $0.000125 y_{75}$ \\
\hline $0.00015625 y_{85}$ & $1.61175 \mathrm{E}-05 y_{85}$ & $0.000125 y_{85}$ \\
\hline $0.000113636 v_{41}$ & $0.000564972 v_{41}$ & $0.00075 v_{41}$ \\
\hline $5.68182 \mathrm{E}-05 v_{51}$ & $0.000564972 v_{51}$ & $0.00075 v_{51}$ \\
\hline $8.92857 \mathrm{E}-05 v_{91}$ & $0.000564972 v_{91}$ & $0.00075 v_{91}$ \\
\hline $0.000138889 v_{101}$ & $0.000564972 v_{101}$ & $0.00075 v_{101}$ \\
\hline \multicolumn{2}{|c|}{ Total Delay $=0.00702 \mathrm{sec}$} \\
\hline
\end{tabular}

Table 4.6: Traffic formulation of the planning example

\begin{tabular}{|c|c|}
\hline Edge Device Traffic $\left(v_{u c}\right)$ & Fog Node Traffic $\left(b_{n l}\right)$ \\
\hline $0 v_{11}$ & $0 b_{11}$ \\
$0 v_{21}$ & $0.28 b_{21}$ \\
$0 v_{31}$ & $0.13 b_{31}$ \\
$16 v_{41}$ & $0 b_{41}$ \\
$33 v_{51}$ & $0.78 b_{51}$ \\
$0 v_{61}$ & \\
$0 v_{71}$ & \\
$0 v_{81}$ & \\
$23 v_{91}$ & \\
$44 v_{101}$ & \\
\hline Total Traffic $=117.19 \mathrm{Mbps}$ \\
\hline
\end{tabular}


The solutions returned by the solver for the three methods was validated by solving the problem manually. Tables 4.5 and 4.6 were used to verify the results of the optimizer.

Since this is a small example, we are able to show partial delay function and traffic function which is exactly equal to the results obtained by the solver.

\subsection{Results Analysis}

The output of the problem is a direct reflection of the input. In the FPP, we are interested in analyzing the effect of increasing the number of edge device clusters and possible fog node placement locations. Typically, with the increase of input size, there will be more delay in the network and more traffic will go towards the cloud. There are different methods of measuring the quality of the solution of an optimization problem. The most common way to measure is to keep track of the objective functions and time taken by the solver to reach the optimal solution.

\subsubsection{Input Parameters}

In this section, we first present the different features of the fog nodes as well as the characteristics of the edge device traffic. All the planning problems are solved with respect to the input specified. Each fog node has a maximum capacity that cannot be crossed. In other words, if one of the requested parameters like memory is greater than the nodes capacity, then the capacity should be increased by using a different fog type or send the edge device cluster request to the cloud. The specification of each fog node depends on the service providers and there are many companies like IBM, Rackspace, Amazon AWS, etc. providing cloud services. Table 4.7 shows the four types of fog nodes that can be used by the solver.

The specifications of the different link types used to connect the fog node(s) to the cloud are shown in Table 4.8. The price of the links depends on their capacity and material which can be obtained from network sales website 77, 78.

The input parameters for the edge device clusters and each edge device within the cluster are tabulated in the tables 4.9 and 4.10 respectively. The simulation area is chosen to be $100 \mathrm{~km}$ by $100 \mathrm{~km}$. This larger area is chosen because we 
Table 4.7: Characteristics of the different fog types

\begin{tabular}{|c|c|c|c|c|c|}
\hline Fog Type & \# of CPU & Memory (GB) & NIC (Mbps) & Cost (\$) & Inventory \\
\hline a & 90 & 480 & 360 & 67200 & 20 \\
\hline b & 180 & 800 & 1024 & 120000 & 15 \\
\hline c & 360 & 1600 & 1024 & 170000 & 10 \\
\hline d & 720 & 3200 & 10240 & 250000 & 4 \\
\hline
\end{tabular}

Table 4.8: Characteristics of the different link types

\begin{tabular}{|c|c|c|}
\hline Link Type & Bandwidth & Cost/meter (\$) \\
\hline a & $100 \mathrm{Mbps}$ & 0.25 \\
\hline b & $1 \mathrm{Gbps}$ & 2 \\
\hline c & $10 \mathrm{Gbps}$ & 200 \\
\hline
\end{tabular}

envision the fog network to be spanning over a city and beyond. Moreover, some point of the simulation there will be 200 edge device clusters which will be hard to accommodate in a smaller area for visual representation. The locations (i.e. the $\mathrm{x}$ and y coordinates) of the edge device clusters and the possible locations are randomly generated within the simulation area. The location cost to install a fog node is set to be $\$ 1000$. The maximum budget of the planning problem was set to $40 \%$ of the maximum cost the fog network can incur. Instead of a fixed value for the budget, we used a percentage of the maximum cost because with the increase of network size (possible placement locations) the maximum cost increases and the budget should also be increased proportionally instead of biasing the small scale problem with a large budget. The cost of a fog network includes the fog node cost, the location cost and the link cost between fog nodes and the cloud (i.e. the hardware cost). The maximum cost includes the installation of highest capacity of fog node(s) in all the optimal locations as well as connecting the fog node(s) and the cloud with the most expensive link type which has the highest speed. The location cost for a fog node depends on many factors. For example, the cost of renting or leasing a location in a commercial area is probably higher than the cost in a residential area. It also depends 
Table 4.9: The edge device cluster input parameters

\begin{tabular}{|c|c|}
\hline Simulation area & $100 \times 100 \mathrm{~km}$ \\
\hline Packet size & 1500 Bytes \\
\hline \# of edge device in a cluster & $\mathrm{U}(10,200)$ \\
\hline
\end{tabular}

Table 4.10: The edge device input parameters for each edge device in a cluster

\begin{tabular}{|c|c|}
\hline \# of CPU core & $\mathrm{U}(1,4)$ \\
\hline Memory & $\mathrm{U}(1,40) \mathrm{GB}$ \\
\hline \# of Packets sent per second & $\mathrm{U}(1,64)$ \\
\hline Network access bandwidth & $\mathrm{U}(20,70) \mathrm{Mbps}$ \\
\hline
\end{tabular}

upon the size and expandibility. To reduce complexity the same cost was used for all the possible fog node placement locations.

Two different sets of problems were solved using three different multiobjective optimization methods. The first set consists of small scale problems and the second set has larger scale problems. For each set, different problem sizes were generated, and for each size, four different instances of the problem were generated. In total, for each mulltiobjective optimization method, we optimized 32 different problem sizes over 4 instances resulting in solving 128 optimization problems. In order to obtain the Pareto front, the weighted sum and the trade-off methods were solved with 11 iterations for each problem (as shown in Figures 4.2 and 4.4 , whereas the hierarchical method was solved with eight iterations for each problem (as explained in Figure 4.3). A time limit of 1.5 hours was set for each iteration. This means that if the optimal solution is not found after 1.5 hours of computation, CPLEX will simply return the best solution obtained so far for that iteration. For each problem, the membership functions 3.31 and 3.32 are used to evaluate each member of the Pareto front and the member with the highest membership function value is extracted as the best compromise result. 


\subsubsection{Small Scale Problems}

In the first set of problems, the number of edge device clusters is varied between 10 and 200 with 5 possible placement locations. 21 different problem sizes (as shown in Table 4.11) are solved over the 4 different instances for a total of 84 problems. The results presented in Table 4.12 are the averages over the 4 different instances (refer to Appendix A for the results of each instance) and includes the best compromised total delay in the network, total traffic to the cloud and the solution time for each multiobjective optimization method. The same CPLEX and hardware configuration was used as described in Section 4.2 .

The first column in Table 4.12 represents the problem number corresponding to the problem size presented in Table 4.11. The second, third and fourth columns represent respectively the total delay in the network, the total traffic going to the cloud and the solution time for the weighted sum optimization method; whereas the fifth, sixth and seventh columns represent respectively the total delay in the network, the total traffic going to the cloud and the solution time for the hierarchical method. Finally, columns 8, 9 and 10 show respectively the total delay in the network, the total traffic going to the cloud and the solution time for the trade-off method.

For the small scale problems, the lowest delay obtained was 0.111 seconds using the hierarchical method and the lowest traffic was 97 Mbps achieved by the weighted sum method.

The delay, traffic and solution time comparisons of the three methods are graphically represented in figures 4.7, 4.8 and 4.9 respectively. For our FPP, we used 95\% Confidence Interval (CI) to examine the reliability of the results obtained. In figures 4.7 and 4.8 , the vertical bars along the graphs show the CI. As it can be seen from the figures, there is a small difference between the three methods in terms of total network delay and traffic towards the cloud. However, the hierarchical method attains less delay and more traffic than the other two methods. This is because the hierarchical optimization is bounded by the delay constraint and we do not allow the objective function to go beyond a reasonable delay in the network which compromises the optimal traffic. With the increase of problem size, the objective functions also increase for all the three methods. This is because more edge device clusters are 
Table 4.11: Problem size for the small scale FPP

\begin{tabular}{|c|c|c|}
\hline Problem & \# of edge device clusters & \# of possible placement locations \\
\hline 1 & 10 & 5 \\
\hline 2 & 15 & 5 \\
\hline 3 & 20 & 5 \\
\hline 4 & 25 & 5 \\
\hline 5 & 30 & 5 \\
\hline 6 & 35 & 5 \\
\hline 7 & 40 & 5 \\
\hline 8 & 45 & 5 \\
\hline 9 & 50 & 5 \\
\hline 10 & 55 & 5 \\
\hline 11 & 60 & 5 \\
\hline 12 & 65 & 5 \\
\hline 13 & 70 & 5 \\
\hline 14 & 75 & 5 \\
\hline 15 & 80 & 5 \\
\hline 16 & 85 & 5 \\
\hline 17 & 90 & 5 \\
\hline 18 & 95 & 5 \\
\hline 19 & 100 & 5 \\
\hline 20 & 150 & 5 \\
\hline 21 & 200 & 5 \\
\hline
\end{tabular}


Table 4.12: Results obtained from the solver for small scale problems (average over 4 instances)

\begin{tabular}{|c|c|c|c|c|c|c|c|c|c|}
\hline & \multicolumn{3}{|c|}{ Weighted Sum } & \multicolumn{3}{|c|}{ Hierarchical } & \multicolumn{3}{|c|}{ Trade-off } \\
\hline Problem & $\begin{array}{c}\text { Delay } \\
(\mathrm{s})\end{array}$ & $\begin{array}{c}\text { Traffic } \\
\text { (Mbps) }\end{array}$ & $\begin{array}{c}\begin{array}{c}\text { Solution Time } \\
(\mathrm{s})\end{array} \\
\end{array}$ & $\begin{array}{c}\text { Delay } \\
(\mathrm{s})\end{array}$ & $\begin{array}{c}\text { Traffic } \\
\text { (Mbps) }\end{array}$ & $\begin{array}{c}\begin{array}{c}\text { Solution Time } \\
(\mathrm{s})\end{array} \\
\end{array}$ & $\begin{array}{c}\text { Delay } \\
(\mathrm{s})\end{array}$ & $\begin{array}{c}\text { Traffic } \\
\text { (Mbps) }\end{array}$ & $\begin{array}{c}\text { Solution Time } \\
(\mathrm{s})\end{array}$ \\
\hline 1 & 0.113 & 97 & 2 & 0.111 & 110 & 3 & 0.113 & 122 & 2 \\
\hline 2 & 0.193 & 175 & 2 & 0.191 & 175 & 4 & 0.193 & 175 & 3 \\
\hline 3 & 0.266 & 401 & 3 & 0.265 & 416 & 5 & 0.266 & 401 & 3 \\
\hline 4 & 0.343 & 391 & 3 & 0.34 & 408 & 7 & 0.343 & 391 & 4 \\
\hline 5 & 0.419 & 604 & 3 & 0.414 & 635 & 8 & 0.42 & 599 & 4 \\
\hline 6 & 0.576 & 919 & 4 & 0.573 & 949 & 12 & 0.576 & 919 & 6 \\
\hline 7 & 0.572 & 898 & 7 & 0.573 & 906 & 16 & 0.572 & 903 & 14 \\
\hline 8 & 0.669 & 1112 & 4 & 0.655 & 1168 & 19 & 0.659 & 1126 & 20 \\
\hline 9 & 0.701 & 1234 & 7 & 0.694 & 1283 & 21 & 0.702 & 1231 & 26 \\
\hline 10 & 0.822 & 1373 & 35 & 0.817 & 1419 & 29 & 0.822 & 1374 & 81 \\
\hline 11 & 0.941 & 1533 & 24 & 0.931 & 1608 & 35 & 0.939 & 1543 & 73 \\
\hline 12 & 0.999 & 1666 & 44 & 0.988 & 1743 & 38 & 0.998 & 1672 & 50 \\
\hline 13 & 1.067 & 1777 & 907 & 1.078 & 1782 & 42 & 1.067 & 1786 & 998 \\
\hline 14 & 1.169 & 1959 & 149 & 1.16 & 2030 & 68 & 1.171 & 1956 & 173 \\
\hline 15 & 1.21 & 2021 & 18 & 1.203 & 2084 & 56 & 1.217 & 1991 & 776 \\
\hline 16 & 1.332 & 2269 & 148 & 1.321 & 2338 & 61 & 1.331 & 2277 & 448 \\
\hline 17 & 1.169 & 2242 & 706 & 1.356 & 2283 & 62 & 1.361 & 2262 & 242 \\
\hline 18 & 1.359 & 2700 & 1010 & 1.354 & 2736 & 76 & 1.36 & 2704 & 438 \\
\hline 19 & 1.617 & 3017 & 25 & 1.602 & 3102 & 74 & 1.613 & 3033 & 139 \\
\hline 20 & 2.398 & 4015 & 513 & 2.392 & 4059 & 211 & 2.401 & 4001 & 365 \\
\hline 21 & 3.22 & 5670 & 3789 & 3.213 & 5706 & 505 & 3.221 & 5672 & 3779 \\
\hline
\end{tabular}




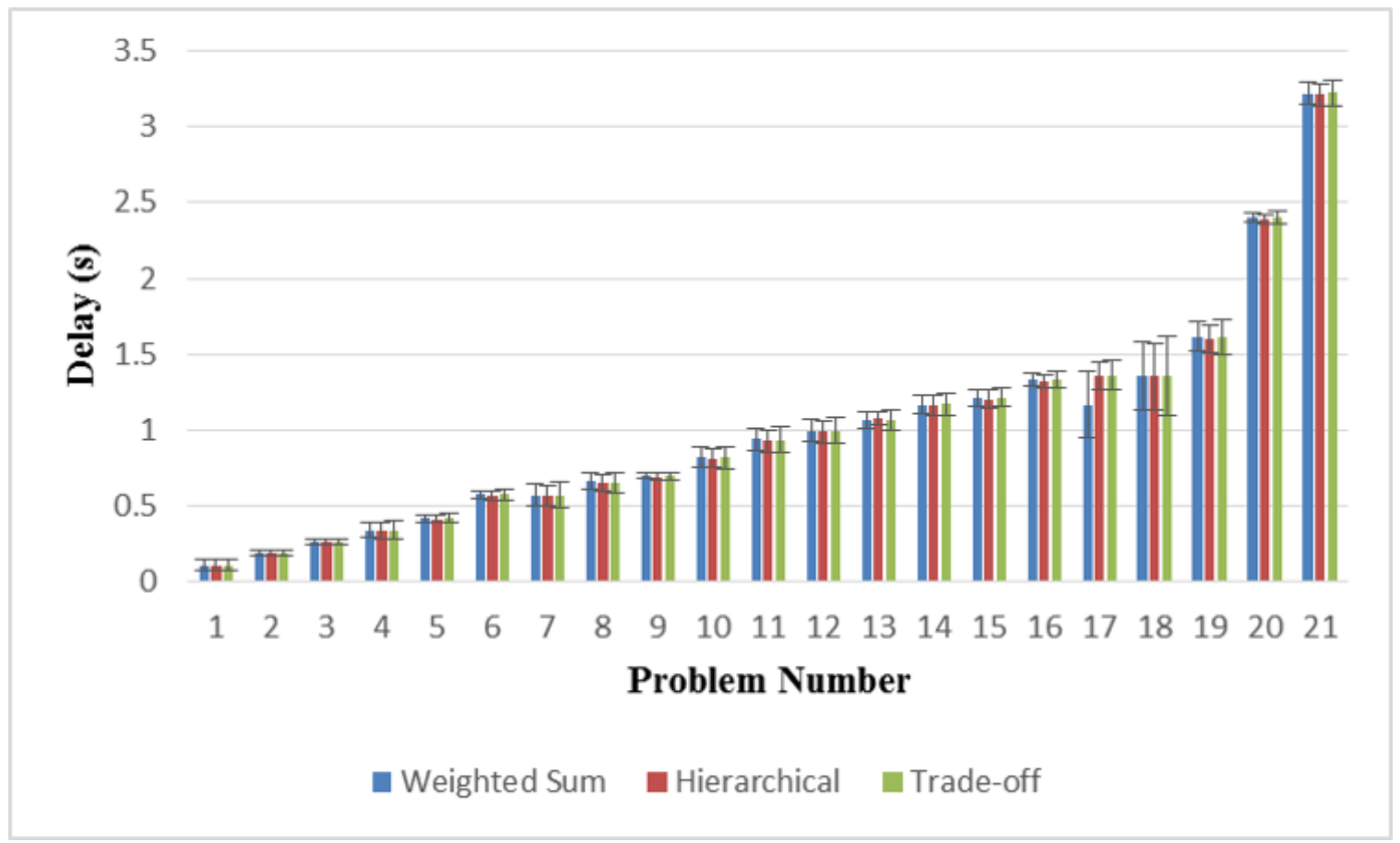

Figure 4.7: Delay comparison with 95\% CI for small scale problems (average over 4 instances)

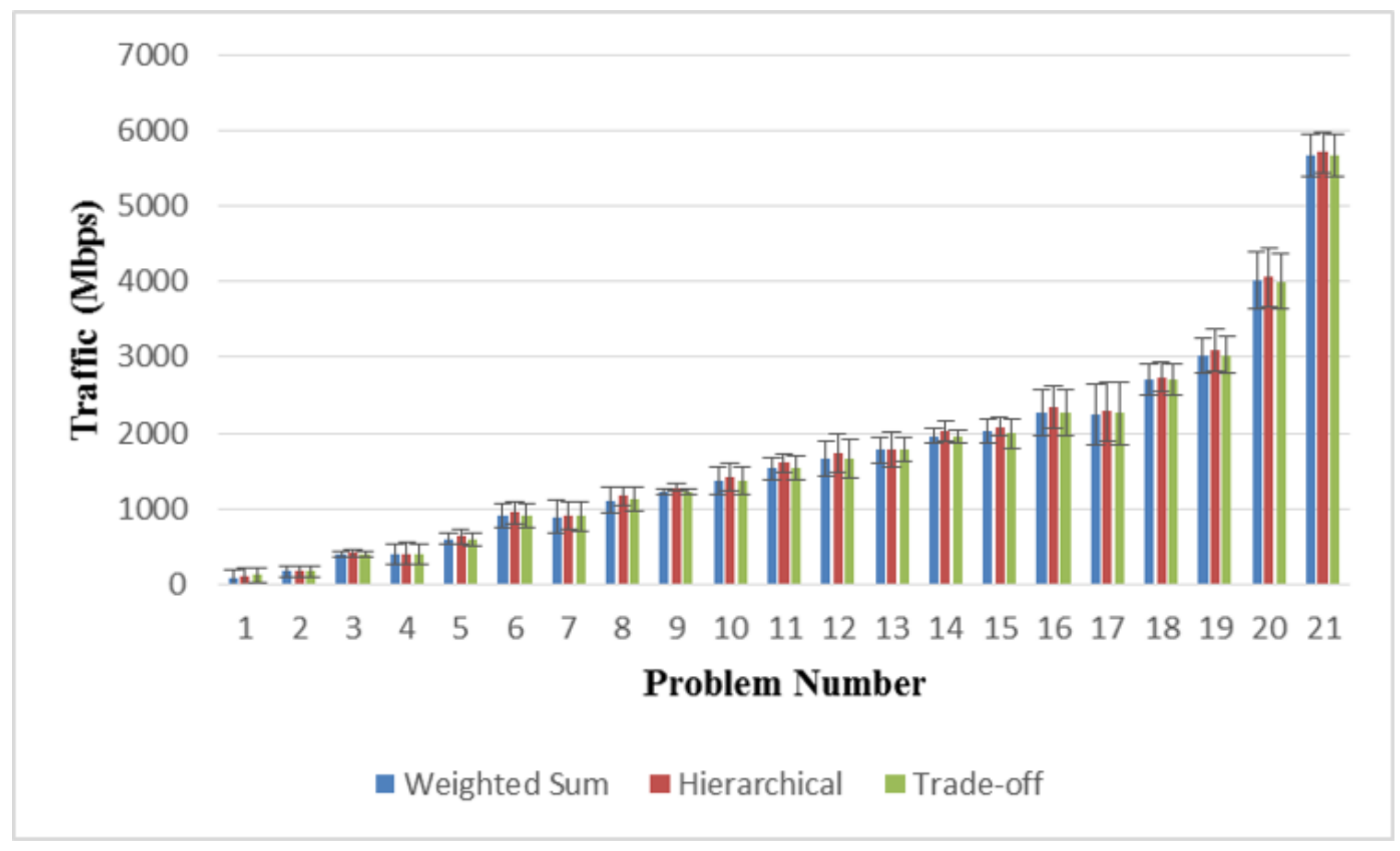

Figure 4.8: Traffic comparison with 95\% CI for small scale problems (average over 4 instances) 


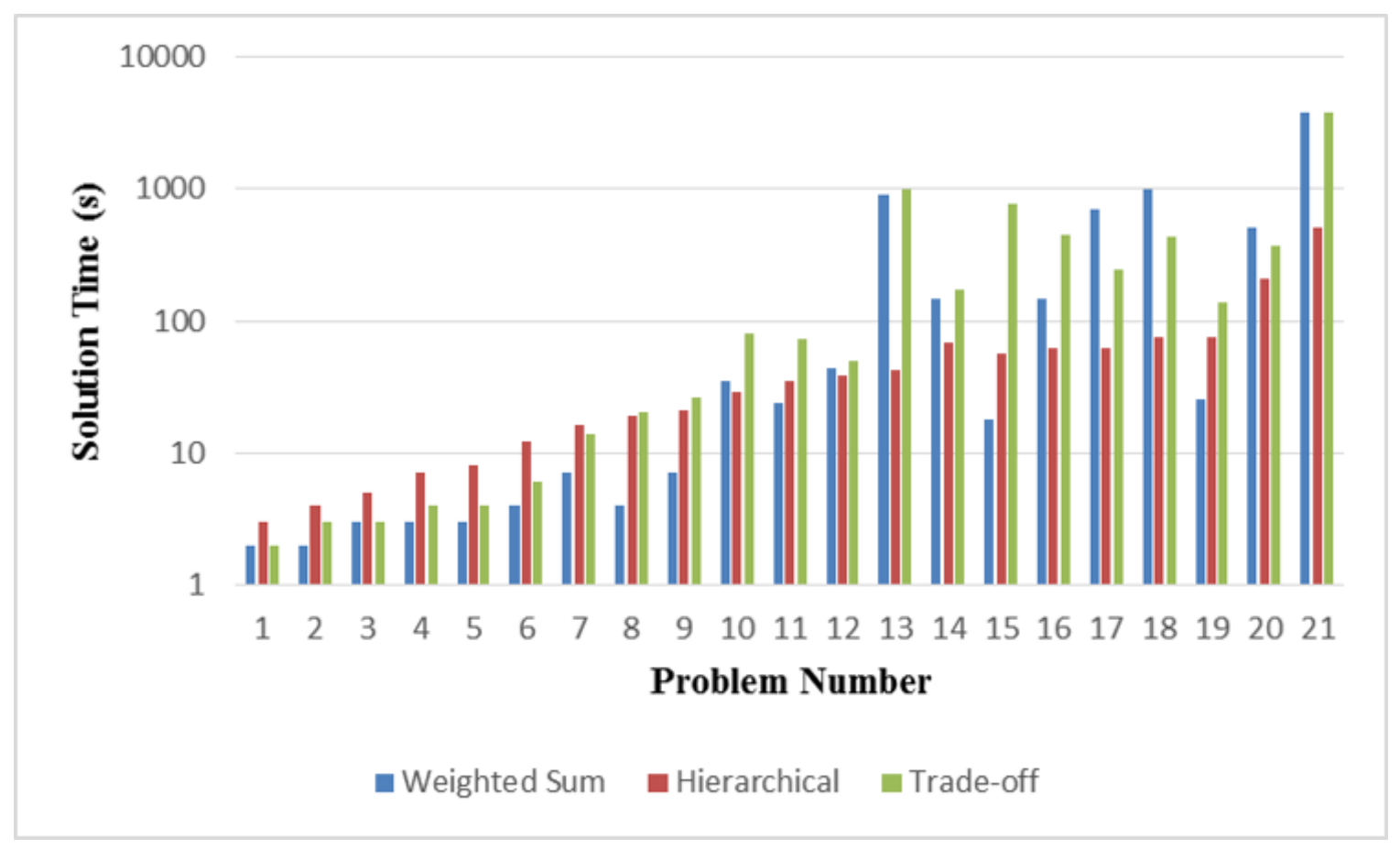

Figure 4.9: Solution time comparison for small scale problems (average over 4 instances)

introduced in the network resulting in more traffic requests from the fog nodes but the number of possible fog node placement location is the same. Hence, many of the edge device clusters are connected to the cloud. The weighted sum method and the trade-off method return similar results for delay and traffic due to the use of the maximum and minimum value of the objective functions as formulated in equations 3.25 and 3.30 .

In general, with the increase of input size, the time taken to obtain an optimal solution must increase as the model must consider a lot of combinations of where to install the fog nodes, what type of fog node needs to be installed and which edge device cluster should be connected to it. From Figure 4.9, it can be seen that the solution time increases in a non linear pattern. This is due to the use of the Branch and Bound (B\&B) algorithm [79] used by the solver [76. In B\&B, at first the search space is recursively split into smaller search spaces called branching and tries to find the minimum objective function in the smaller search space. To avoid the brute-force search and testing all the candidate solutions, the algorithm uses heuristic to keep track of bounds on the minimum that it is trying to find and these bounds are used to cut back the search space by eliminating the candidate solutions which cannot give 
optimal solution. To add with that, for the same problem, different multiobjective optimization methods generate different search spaces. For instance, from problem seven onward, the hierarchical method takes a lot less time compared to at least one of the methods and in some cases both methods. The reason it takes less time is because the objective function is traffic towards the cloud which according to Equation 3.6 has less variables and constraint 3.29 provides a strong feasible initial solution for the particular method compared to the other two optimization methods. Moreover, for each problem, the hierarchical method runs for 8 iterations which is 3 less than the other two methods. Although the CI for the average solution time was calculated, it could not be represented in the figure because of the semi-log scale used in the plot.

\section{Comparison to the Best}

Table 4.13 show the comparison of the results among the three multiobjective optimization methods for small scale problems. The first column represents the problem number. The second and third columns illustrate the percentage gap for the delay and traffic between the weighted sum method and the minimum value obtained from all three methods. Similarly, columns 4 and 5 show the percentage gap for the delay and traffic between the hierarchical method and the minimum value achieved from all three methods. Finally, columns 6 and 7 represent the delay and traffic percentage difference between the trade-off method and the minimum objective function value returned by all three methods. A $0 \%$ implies that the delay or traffic value obtained by that method for the particular problem is the minimum among all the three methods, hence, there is no percentage difference. The mean percentage gap for the weighted sum method for the delay and traffic is $0.73 \%$ and $0.15 \%$ respectively. The mean of the delay gap for the hierarchical method is $0.82 \%$ and the traffic is $3.49 \%$. For the trade-off method, the mean of the delay gap obtained was $1.46 \%$, whereas, the mean of the traffic difference was $1.51 \%$. From the results, it can be concluded that, for small scale problems, the weighted sum method returns the best optimal results among the three methods. 
Table 4.13: Solutions comparison among the three methods over 4 instances

\begin{tabular}{|c|c|c|c|c|c|c|}
\hline & \multicolumn{2}{|c|}{ Weighted Sum } & \multicolumn{2}{|c|}{ Hierarchical } & \multicolumn{2}{|c|}{ Trade-off } \\
\hline Problem & $\begin{array}{c}\text { Delay Gap } \\
(\%)\end{array}$ & $\begin{array}{c}\text { Traffic Gap } \\
(\%)\end{array}$ & $\begin{array}{c}\text { Delay Gap } \\
(\%)\end{array}$ & $\begin{array}{c}\text { Traffic Gap } \\
(\%)\end{array}$ & $\begin{array}{c}\text { Delay Gap } \\
(\%)\end{array}$ & $\begin{array}{c}\text { Traffic Gap } \\
(\%)\end{array}$ \\
\hline 1 & 1.5 & 0 & 0 & 13 & 1.4 & 26 \\
\hline 2 & 1 & 0.2 & 0 & 0 & 1 & 0.2 \\
\hline 3 & 0.65 & 0 & 0 & 3.8 & 0.65 & 0 \\
\hline 4 & 0.89 & 0 & 0 & 4.2 & 0.89 & 0 \\
\hline 5 & 1.1 & 0.81 & 0 & 6 & 1.5 & 0 \\
\hline 6 & 0.53 & 0 & 0 & 3.3 & 0.53 & 0.058 \\
\hline 7 & 0.022 & 0 & 0.061 & 0.82 & 0 & 0.57 \\
\hline 8 & 2.1 & 0 & 0 & 5.1 & 0.68 & 1.3 \\
\hline 9 & 0.9 & 0.22 & 0 & 4.2 & 1.1 & 0 \\
\hline 10 & 0.53 & 0 & 0 & 3.4 & 0.53 & 0.092 \\
\hline 11 & 1 & 0 & 0 & 4.9 & 0.84 & 0.66 \\
\hline 12 & 1.1 & 0 & 0 & 4.7 & 0.95 & 0.36 \\
\hline 13 & 0.033 & 0 & 1.1 & 0.28 & 0 & 0.48 \\
\hline 14 & 0.8 & 0.12 & 0 & 3.8 & 0.92 & 0 \\
\hline 15 & 0.54 & 1.5 & 0 & 4.7 & 1.2 & 0 \\
\hline 16 & 0.8 & 0 & 0 & 3 & 0.78 & 0.38 \\
\hline 17 & 0 & 0 & 16 & 1.9 & 16 & 0.92 \\
\hline 18 & 0.34 & 0 & 0 & 1.3 & 0.41 & 0.16 \\
\hline 19 & 0.92 & 0 & 0 & 2.8 & 0.73 & 0.52 \\
\hline 20 & 0.25 & 0.35 & 0 & 1.4 & 0.38 & 0 \\
\hline 21 & 0.22 & 0 & 0 & 0.62 & 0.25 & 0.035 \\
\hline Mean & 0.73 & 0.15 & 0.82 & 3.49 & 1.46 & 1.51 \\
\hline
\end{tabular}


Table 4.14: Solutions comparison between single objective and multiobjective (over 4 instances)

\begin{tabular}{|c|c|c|c|c|c|c|c|c|}
\hline & \multicolumn{2}{|c|}{ Single Objective } & \multicolumn{2}{|c|}{ Weighted Sum } & \multicolumn{2}{|c|}{ Hierarchical } & \multicolumn{2}{|c|}{ Trade-off } \\
\hline Problem & $\begin{array}{l}\text { Delay } \\
(\mathrm{s})\end{array}$ & $\begin{array}{l}\text { Traffic } \\
\text { (Mbps) }\end{array}$ & $\begin{array}{c}\text { Delay Gap } \\
(\%)\end{array}$ & $\begin{array}{c}\text { Traffic Gap } \\
(\%)\end{array}$ & $\begin{array}{c}\text { Delay Gap } \\
(\%)\end{array}$ & $\begin{array}{c}\text { Traffic Gap } \\
(\%)\end{array}$ & $\begin{array}{c}\text { Delay Gap } \\
(\%)\end{array}$ & $\begin{array}{c}\text { Traffic Gap } \\
(\%)\end{array}$ \\
\hline 1 & 0.111 & 95 & 1.8 & 2.1 & 0 & 16 & 1.8 & 28 \\
\hline 2 & 0.191 & 163 & 1 & 7.4 & 0 & 7.4 & 1 & 7.4 \\
\hline 3 & 0.265 & 390 & 0.38 & 2.8 & 0 & 6.7 & 0.38 & 2.8 \\
\hline 4 & 0.34 & 370 & 0.88 & 5.7 & 0 & 10 & 0.88 & 5.7 \\
\hline 5 & 0.414 & 570 & 1.2 & 6 & 0 & 11 & 1.4 & 5.1 \\
\hline 6 & 0.573 & 899 & 0.52 & 2.2 & 0 & 5.6 & 0.52 & 2.2 \\
\hline 7 & 0.566 & 851 & 1.1 & 5.5 & 1.2 & 6.5 & 1.1 & 6.1 \\
\hline 8 & 0.655 & 1070 & 2.1 & 3.9 & 0 & 9.2 & 0.61 & 5.2 \\
\hline 9 & 0.694 & 1197 & 1 & 3.1 & 0 & 7.2 & 1.2 & 2.8 \\
\hline 10 & 0.817 & 1322 & 0.61 & 3.9 & 0 & 7.3 & 0.61 & 3.9 \\
\hline 11 & 0.931 & 1484 & 1.1 & 3.3 & 0 & 8.4 & 0.86 & 4 \\
\hline 12 & 0.988 & 1619 & 1.1 & 2.9 & 0 & 7.7 & 1 & 3.3 \\
\hline 13 & 1.058 & 1712 & 0.85 & 3.8 & 1.9 & 4.1 & 0.85 & 4.3 \\
\hline 14 & 1.158 & 1903 & 0.95 & 2.9 & 0.17 & 6.7 & 1.1 & 2.8 \\
\hline 15 & 1.199 & 1936 & 0.92 & 4.4 & 0.33 & 7.6 & 1.5 & 2.8 \\
\hline 16 & 1.319 & 2184 & 0.99 & 3.9 & 0.15 & 7.1 & 0.91 & 4.3 \\
\hline 17 & 1.158 & 2174 & 0.95 & 3.1 & 17 & 5 & 18 & 4 \\
\hline 18 & 1.351 & 2647 & 0.59 & 2 & 0.22 & 3.4 & 0.67 & 2.2 \\
\hline 19 & 1.596 & 2962 & 1.3 & 1.9 & 0.38 & 4.7 & 1.1 & 2.4 \\
\hline 20 & 2.383 & 3919 & 0.63 & 2.4 & 0.38 & 3.6 & 0.76 & 2.1 \\
\hline 21 & 3.2 & 5592 & 0.63 & 1.4 & 0.41 & 2 & 0.66 & 1.4 \\
\hline \multicolumn{3}{|c|}{ Mean } & 0.98 & 3.55 & 2.21 & 7 & 1.76 & 4.90 \\
\hline
\end{tabular}




\section{Comparison to Single Objective Function for Small Scale Problems}

Until now, we compared the optimal solutions and the time taken in obtaining the solution for different small scale problems but this does not explain the reliability of the methods and their Pareto front. There are different ways to measure the reliability of multiobjective optimization models. One of the ways is to optimize each objective function individually in order to study the diversity characteristics of the trade-off surface in a Pareto front. Table 4.14 represents a comparison between the results of single objective optimization and multiobjective optimization for 21 problems over the 4 different instances (refer to Appendix $\mathrm{C}$ for the results of each instance). The first column in the table represents the problem number. The optimal delay and traffic for each problem when optimized individually are given in columns 2 and 3. Columns 4, 5, 6, 7, 8 and 9 represent the percentage difference between the individual single objective optimal result and the compromised optimal values obtained using the three multiobjective optimization methods shown in Table 4.12. The mean difference in optimal delay for the weighted sum method is $0.98 \%$ and the traffic is $3.55 \%$. For the hierarchical method, the mean difference in delay and traffic for all the small scale problems is $2.21 \%$ and $7 \%$ respectively. Whereas, the trade-off method attained a mean difference of $1.76 \%$ for optimal delay and $4.9 \%$ for optimal traffic compared to individual optimization. For half of the problems, the optimal delay obtained by the hierarchical method is exactly the same as the individually optimized delay but the difference in traffic is high compare to the other two methods. This is due to the tight delay constraint which ensures minimal sacrifice of optimal delay in the network. Overall, the difference in each of the result is not more than $7 \%$, which shows that for small scale problems, all of the three methods are able to solve multiobjective functions, returning nearly the same result as the single objective optimization for each objective function.

\section{The Hypervolume Indicator}

The hypervolume indicator is another widely used performance metric for multiobjective optimization. Figure 4.10 shows the HV comparison between the three methods for the small scale problems. For different problem sizes we obtain different HV ranging from a lowest value of 0.274 to a highest value of 0.783 . From the figure, it can be observed that the hierarchical method results in smaller HV compared to the other two methods. This is due to the lack of non-dominated member in the 


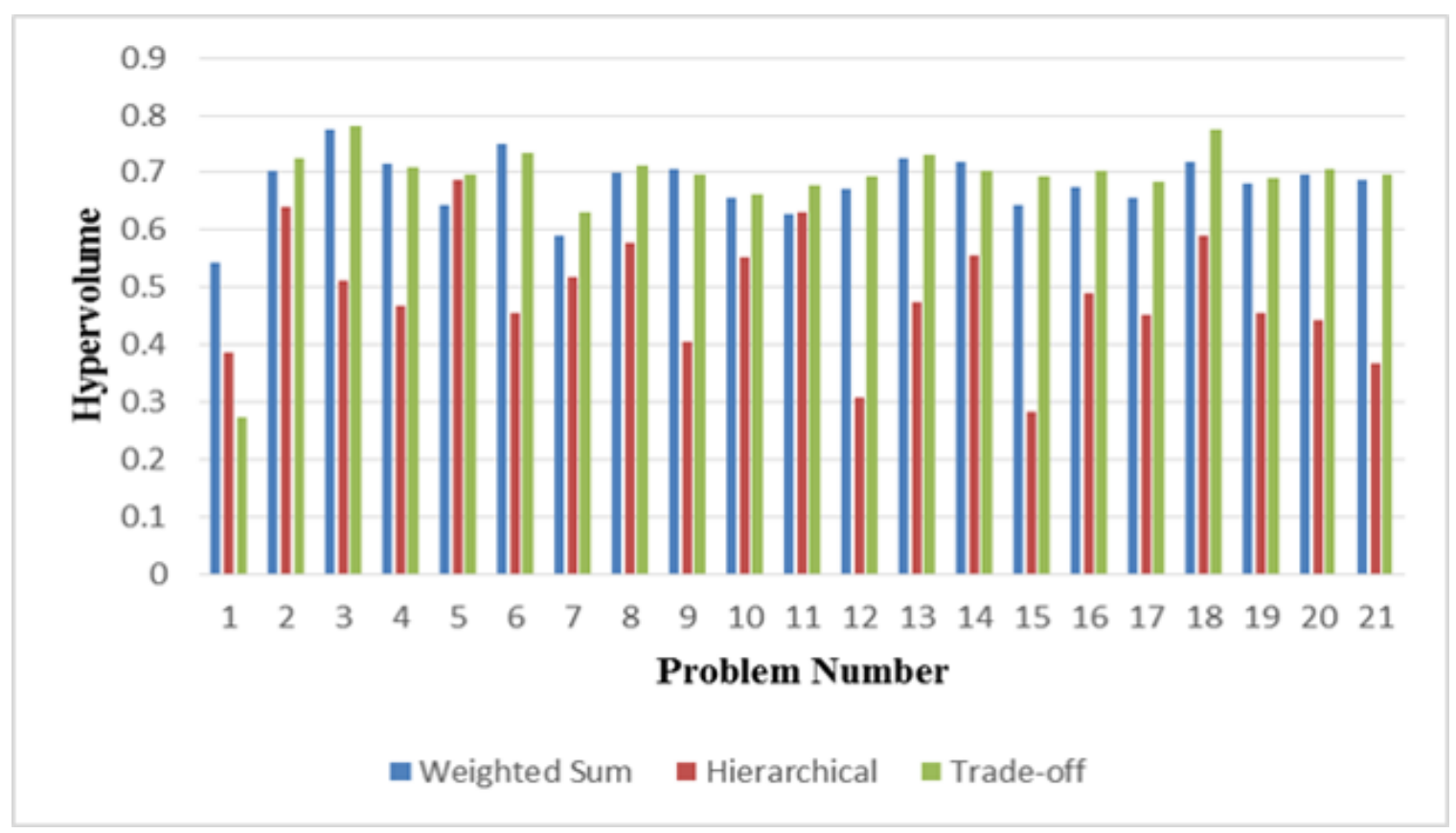

Figure 4.10: Average HV indicator for small scale problems

Pareto front, as the hierarchical method can at most generate only eight points or less in the Pareto front. Moreover, the delay constraint does not allow to explore as many combinations as the other two methods can do. The weighted sum method and the trade-off method have similar HV for its respective Pareto front for each problem. However, the trade-off method outperforms the weighted sum method by a slight margin proving that it has more diverse non-dominated solutions and better convergence towards the true approximated Pareto front among all the three methods.

The following section explores the effect of increasing the problem size to a larger scale.

\subsubsection{Large scale problems}

The second set of problems shows how the three methods behave when the number of possible placement locations is increased. The number of possible placement locations is increased from 5 to 10 for a range between 10 to 60 edge device clusters (see Table 4.15). The number of edge device cluster is stopped at 60 because after 45 edge device clusters, optimal solutions were not guaranteed as several iterations 
Table 4.15: Problem size for the large scale FPP

\begin{tabular}{|c|c|c|}
\hline Problem & \# of edge device clusters & \# of possible placement locations \\
\hline 22 & 10 & 10 \\
\hline 23 & 15 & 10 \\
\hline 24 & 20 & 10 \\
\hline 25 & 25 & 10 \\
\hline 26 & 30 & 10 \\
\hline 27 & 35 & 10 \\
\hline 28 & 40 & 10 \\
\hline 29 & 45 & 10 \\
\hline 30 & 50 & 10 \\
\hline 31 & 55 & 10 \\
\hline 32 & 60 & 10 \\
\hline
\end{tabular}

where not returning the optimal solution within the time limit. A total of 44 problems were solved with 11 different problem sizes and the average of each problem size was used for the analysis of the problem set (refer to Appendix B for the results of all instances). We used the same CPLEX and hardware environment as defined for the example problem.

The first column in Table 4.16 represents the problem number with respect to the problem size presented in Table 4.15. The second, third and fourth columns represent respectively the total delay in the network, the total traffic going to the cloud and the solution time for the weighted sum optimization method; whereas the fifth, sixth and seventh columns represent respectively the total delay in the network, the total traffic going to the cloud and the solution time for the hierarchical method. Finally, columns 8,9 and 10 show respectively the total delay in the network, the total traffic going to the cloud and the solution time for the trade-off method. It is important to note that some of the iterations could not find the optimal value within the time limit. Although these solutions are not optimal, they were still included in the Pareto front. The fuzzy-based mechanism which was used to find the best compromise result always returned the membership value which has been solved within the time limit. 
Table 4.16: Results obtained from the solver for large scale problems (average over 4 instances)

\begin{tabular}{|c|c|c|c|c|c|c|c|c|c|}
\hline & \multicolumn{3}{|c|}{ Weighted Sum } & \multicolumn{3}{c|}{ Hierarchical } & \multicolumn{3}{c|}{ Trade-off } \\
\hline \hline Problem & $\begin{array}{c}\text { Delay } \\
(\mathbf{s})\end{array}$ & $\begin{array}{c}\text { Traffic } \\
(\mathrm{Mbps})\end{array}$ & $\begin{array}{c}\text { Solution Time } \\
(\mathbf{s})\end{array}$ & $\begin{array}{c}\text { Delay } \\
(\mathbf{s})\end{array}$ & $\begin{array}{c}\text { Traffic } \\
(\mathrm{Mbps})\end{array}$ & $\begin{array}{c}\text { Solution Time } \\
(\mathbf{s})\end{array}$ & $\begin{array}{c}\text { Delay } \\
(\mathrm{s})\end{array}$ & $\begin{array}{c}\text { Traffic } \\
(\mathrm{Mbps})\end{array}$ & $\begin{array}{c}\text { Solution Time } \\
(\mathbf{s})\end{array}$ \\
\hline \hline 22 & 0.119 & 127 & 2 & 0.119 & 127 & 5 & 0.119 & 129 & 2 \\
\hline 23 & 0.197 & 157 & 19 & 0.196 & 164 & 13 & 0.197 & 166 & 6 \\
\hline 24 & 0.261 & 226 & 258 & 0.259 & 235 & 124 & 0.261 & 237 & 2700 \\
\hline 25 & 0.341 & 355 & 6020 & 0.34 & 371 & 807 & 0.343 & 344 & 10445 \\
\hline 26 & 0.449 & 607 & 1230 & 0.444 & 630 & 320 & 0.447 & 613 & 936 \\
\hline 27 & 0.492 & 576 & 1187 & 0.494 & 627 & 2504 & 0.495 & 612 & 801 \\
\hline 28 & 0.589 & 713 & 2110 & 0.579 & 785 & 1753 & 0.585 & 731 & 946 \\
\hline 29 & 0.691 & 840 & 4612 & 0.687 & 908 & 4383 & 0.691 & 845 & 6414 \\
\hline 30 & 0.666 & 897 & 2001 & 0.66 & 972 & 2837 & 0.664 & 924 & 1615 \\
\hline 31 & 0.783 & 944 & 6063 & 0.769 & 1058 & 3359 & 0.783 & 950 & 6080 \\
\hline 32 & 0.876 & 1195 & 12054 & 0.856 & 1254 & 9009 & 0.864 & 1210 & 8370 \\
\hline
\end{tabular}

As it is for a small number of problems, we cannot make sure that this will always be the case.

All the three methods returned a lowest delay value of 0.119 seconds, whereas the weiighted sum method and the hierarchical method attained the lowest traffic value of 127 Mbps.

Figure 4.11 graphically represents the delay comparison between the three methods for large scale problems over four different instances with a 95\% CI. As it can be noticed from the figure, as the problem size increases, the delay in the network also increases. We can observe a linearity in the increment of delay for problems 22 to 29 , but the pattern is disrupted by problem 30 for which the delay in the network is less than the previous problem size. The pattern continues for problem 31 and 32. However, we believe that, if we run each problem for more than four instances, a more linear pattern can be seen without any interruption.

The traffic comparison between the three methods for the four different instances of the second problem set is illustrated in Figure 4.12. The vertical bars on the columns show a $95 \%$ CI for each problem. The traffic obtained from the weighted sum and trade-off are very similar, whereas the hierarchical method attains a slightly higher traffic for every problem. This is because, in the FPP, the hierarchical method 


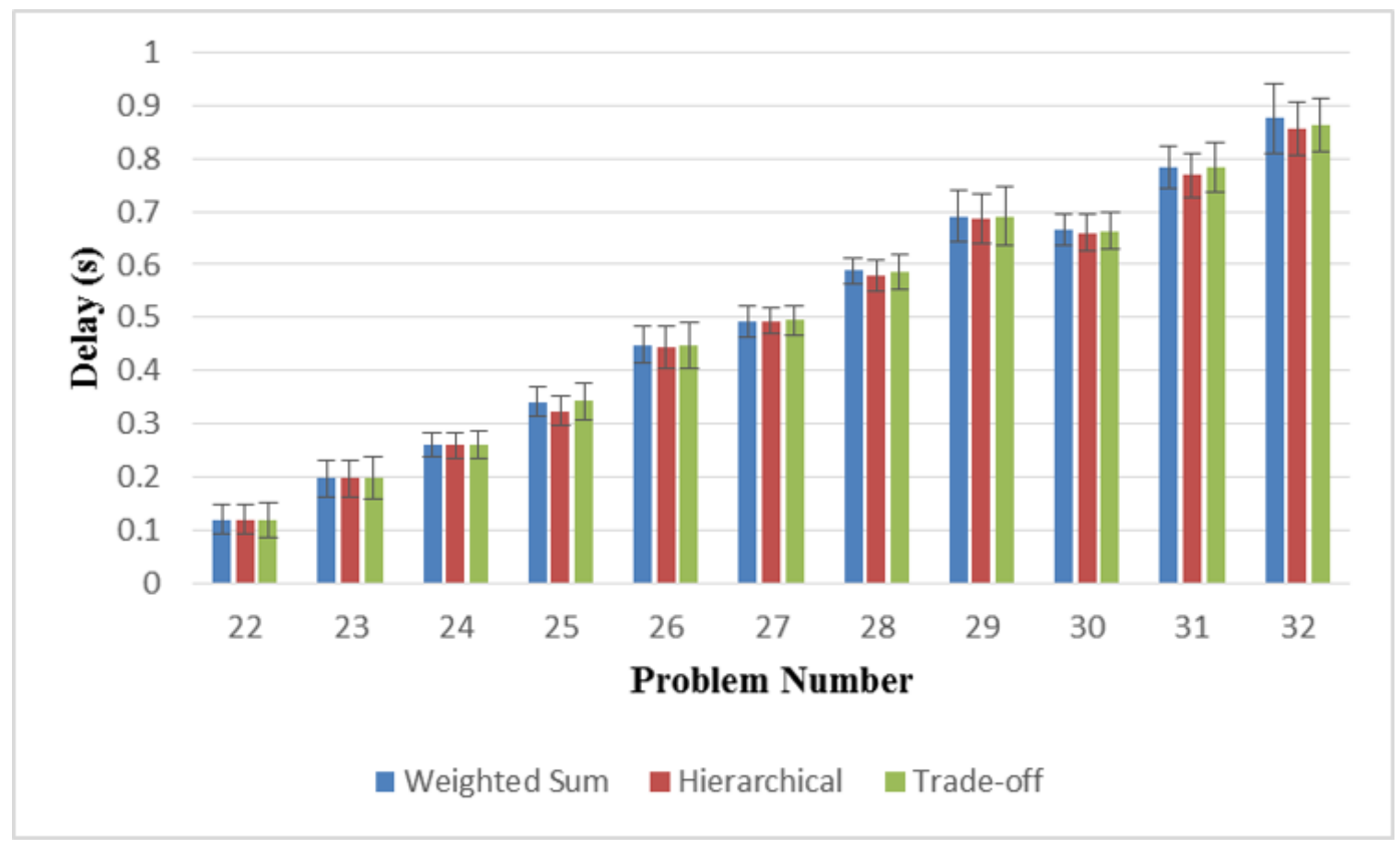

Figure 4.11: Delay comparison with 95\% CI for large scale problems (average over 4 instances)

was proposed with respect to a delay sensitive network. It can be clearly observed from the figure, that the traffic in the cloud increases linearly with the problem size for first 5 problems. However, problem 27 interrupts the pattern. The pattern again continues for the last 5 problems. If the number of instances is increased a smoother pattern can be observed.

The average solution times are illustrated in Figure 4.13 for the three methods over four instances. As the vertical axis in the figure is in logarithmic scale, the calculated CI could not be illustrated. The figure shows that there is a non linear increasing pattern for the average solution time for all the problems. The randomness in the solution time relates to the theory that the combinational space are shortened by the solver using heuristics. As the figure depicts, for the same problem size there is a visible difference in solution time for the three methods. For example, to solve problem 25 , the weighted sum method takes 6020 seconds, the hierarchical method solves in 807 seconds and the trade-off method takes 10445 seconds. Each method generates a different search space for the same problem, hence solving the same problem size 


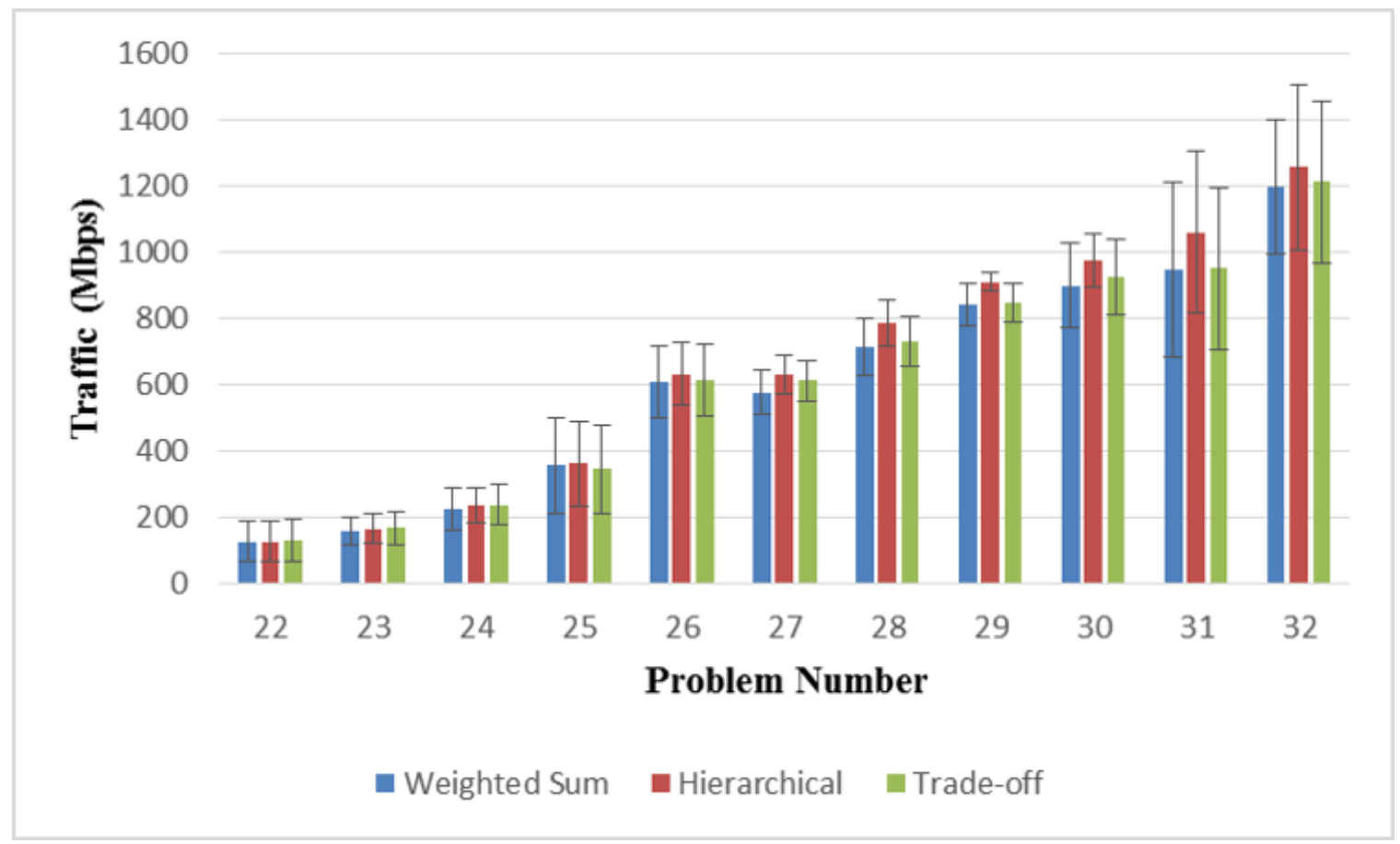

Figure 4.12: Traffic comparison with 95\% CI for large scale problems (average over 4 instances)

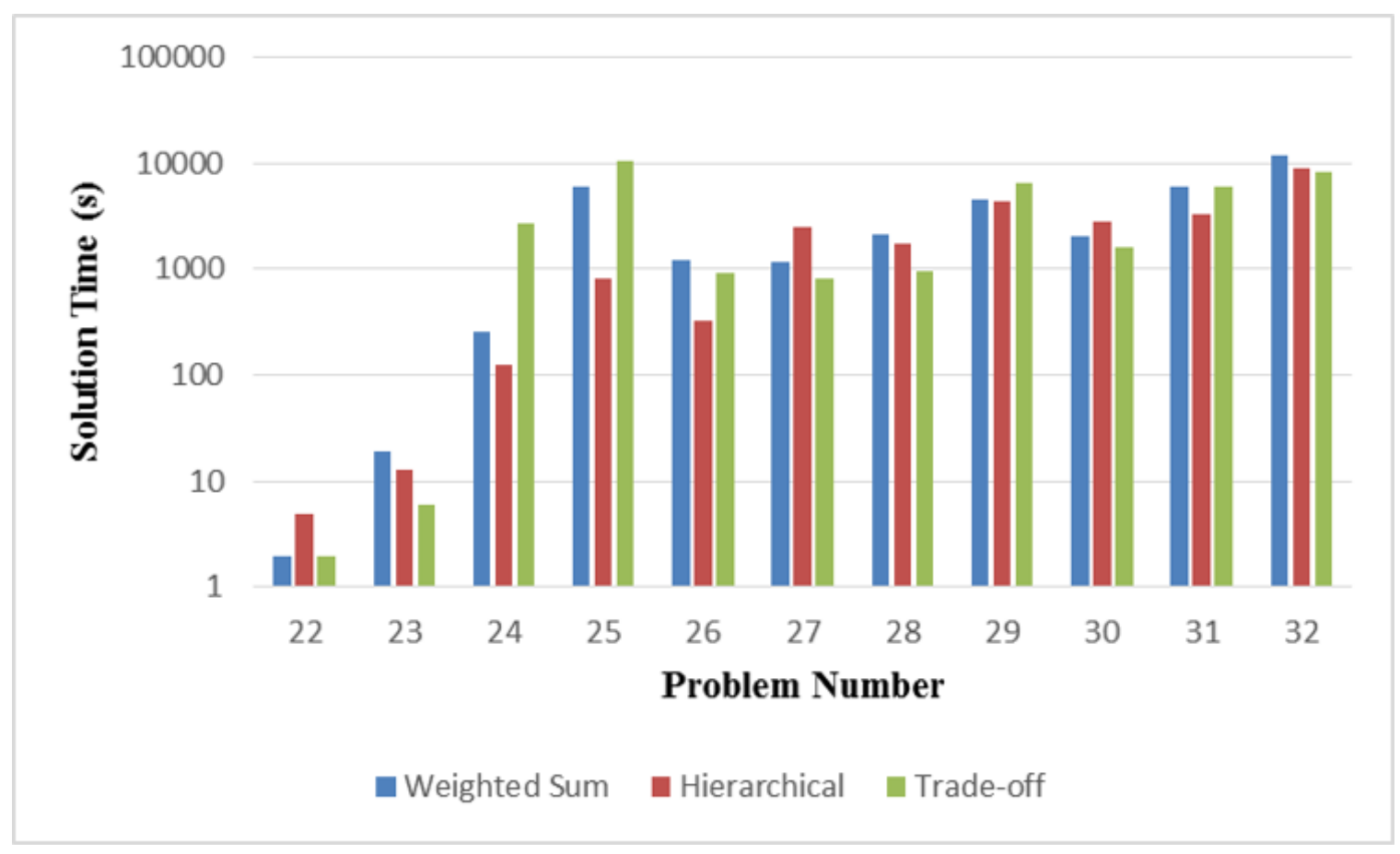

Figure 4.13: Solution time comparison for large scale problems (average over 4 instances) 
Table 4.17: Average solution comparison among the three methods over 4 instances for large scale problems

\begin{tabular}{|c|c|c|c|c|c|c|}
\hline & \multicolumn{2}{|c|}{ Weighted Sum } & \multicolumn{2}{c|}{ Hierarchical } & \multicolumn{2}{c|}{ Trade-off } \\
\hline \hline Problem & $\begin{array}{c}\text { Delay Gap } \\
(\%)\end{array}$ & $\begin{array}{c}\text { Traffic Gap } \\
(\%)\end{array}$ & $\begin{array}{c}\text { Delay Gap } \\
(\%)\end{array}$ & $\begin{array}{c}\text { Traffic Gap } \\
(\%)\end{array}$ & $\begin{array}{c}\text { Delay Gap } \\
(\%)\end{array}$ & $\begin{array}{c}\text { Traffic Gap } \\
(\%)\end{array}$ \\
\hline \hline 22 & 0.013 & 0 & 0.019 & 0 & 0 & 1.2 \\
\hline 23 & 0.74 & 0 & 0 & 4.3 & 0.67 & 5.7 \\
\hline 24 & 0.55 & 0 & 0 & 4.1 & 0.44 & 5 \\
\hline 25 & 0.56 & 3.3 & 0 & 7.7 & 0.96 & 0 \\
\hline 26 & 1 & 0 & 0 & 3.7 & 0.68 & 1 \\
\hline 27 & 0 & 0 & 0.22 & 8.7 & 0.57 & 6.2 \\
\hline 28 & 1.6 & 0 & 0 & 10 & 1.1 & 2.6 \\
\hline 29 & 0.67 & 0 & 0 & 8.1 & 0.63 & 0.56 \\
\hline 30 & 0.94 & 0 & 0 & 8.3 & 0.65 & 3 \\
\hline 31 & 1.9 & 0 & 0 & 12 & 1.9 & 0.65 \\
\hline 32 & 2.3 & 0 & 0 & 4.9 & 0.94 & 1.2 \\
\hline Mean & $\mathbf{0 . 9 3}$ & $\mathbf{0 . 3}$ & $\mathbf{0 . 0 2 2}$ & $\mathbf{6 . 5 3}$ & $\mathbf{0 . 7 8}$ & $\mathbf{2 . 4 6}$ \\
\hline
\end{tabular}

with different solution time.

\section{Comparison to the Best}

Table 4.17 represents the comparison of results among the three multiobjective optimization methods for large scale problems. Column 1 in the table shows the problem number. The second and third columns show the percentage difference for the average delay and traffic between the weighted sum method and the minimum value obtained from all three methods. Columns 4 and 5 represent the percentage gap for the average delay and traffic between the hierarchical method and the minimum value achieved from all three methods. Whereas, columns 6 and 7 illustrate the average delay and traffic percentage difference between the trade-off method and the minimum objective function value returned by all three methods. Although, the hierarchical method returns the best results for the delay value in the network, the weighted sum method performs the best when both objectives are considered, with a lowest mean percentage difference of $1.23 \%$ when both objectives are combined. 
Table 4.18: Solutions comparison between single objective and multiobjective over 4 instances for large scale problems

\begin{tabular}{|c|c|c|c|c|c|c|c|c|}
\hline & \multicolumn{2}{|c|}{ Single Objective } & \multicolumn{2}{|c|}{ Weighted Sum } & \multicolumn{2}{c|}{ Hierarchical } & \multicolumn{2}{c|}{ Trade-off } \\
\hline \hline Problem & $\begin{array}{c}\text { Delay } \\
(\mathbf{s})\end{array}$ & $\begin{array}{c}\text { Traffic } \\
(\mathbf{M b p s})\end{array}$ & $\begin{array}{c}\text { Delay Gap } \\
(\mathbf{\%})\end{array}$ & $\begin{array}{c}\text { Traffic Gap } \\
(\mathbf{\%})\end{array}$ & $\begin{array}{c}\text { Delay Gap } \\
(\mathbf{\%})\end{array}$ & $\begin{array}{c}\text { Traffic Gap } \\
(\%)\end{array}$ & $\begin{array}{c}\text { Delay Gap } \\
(\%)\end{array}$ & $\begin{array}{c}\text { Traffic Gap } \\
(\%)\end{array}$ \\
\hline \hline 22 & 0.119 & 127 & 0 & 0 & 0 & 0 & 0 & 1.6 \\
\hline 23 & 0.196 & 154 & 0.51 & 1.9 & 0 & 6.5 & 0.51 & 7.8 \\
\hline 24 & 0.259 & 224 & 0.77 & 0.89 & 0 & 4.9 & 0.77 & 5.8 \\
\hline 25 & 0.339 & 316 & 0.59 & 12 & 0.29 & 17 & 1.2 & 8.9 \\
\hline 26 & 0.444 & 582 & 1.1 & 4.3 & 0 & 8.2 & 0.68 & 5.3 \\
\hline 27 & 0.493 & 552 & 0.2 & 4.3 & 0.2 & 14 & 0.41 & 11 \\
\hline 28 & 0.579 & 679 & 1.7 & 5 & 0 & 16 & 1 & 7.7 \\
\hline 29 & 0.686 & 784 & 0.73 & 7.1 & 0.15 & 16 & 0.73 & 7.8 \\
\hline 30 & 0.659 & 836 & 1.1 & 7.3 & 0.15 & 16 & 0.76 & 11 \\
\hline 31 & 0.768 & 874 & 2 & 8 & 0.13 & 21 & 2 & 8.7 \\
\hline 32 & 0.853 & 1144 & 2.7 & 4.5 & 0.35 & 9.6 & 1.3 & 5.8 \\
\hline & Mean & & $\mathbf{1 . 1 4}$ & $\mathbf{5 . 5 3}$ & $\mathbf{0 . 2 1}$ & $\mathbf{1 2 . 9 2}$ & $\mathbf{0 . 9 4}$ & $\mathbf{7 . 4}$ \\
\hline
\end{tabular}

\section{Comparison to Single Objective Function for Large Scale Problems}

To understand the reliability of the three methods for large scale problems, the two objective functions were optimized individually. The average results over the 4 different instances (refer to Appendix C for the results of each instance) are tabulated in Table 4.18 and compared with the multiobjective optimization results. In the table, column 1 represents the problem number referring to the the problem size from Table 4.15. The delay and traffic for each individually optimized problem is given in columns 2 and 3 respectively. Columns 4 and 5 show the delay and traffic gap between the weighted sum method and the single objective. The delay and traffic gap for the hierarchical method are given in columns 6 and 7. Finally, the eighth and ninth columns show the gap obtained from the trade-off method.

The weighted sum method returns a combined lowest percentage gap of $6.67 \%$ among the three methods. Overall, the percentage gap are very reasonable considering the trade-off, summarizing that for large scale FPP, the proposed methods can return multiobjective optimized solution as the near optimal solution of single objective functions. 


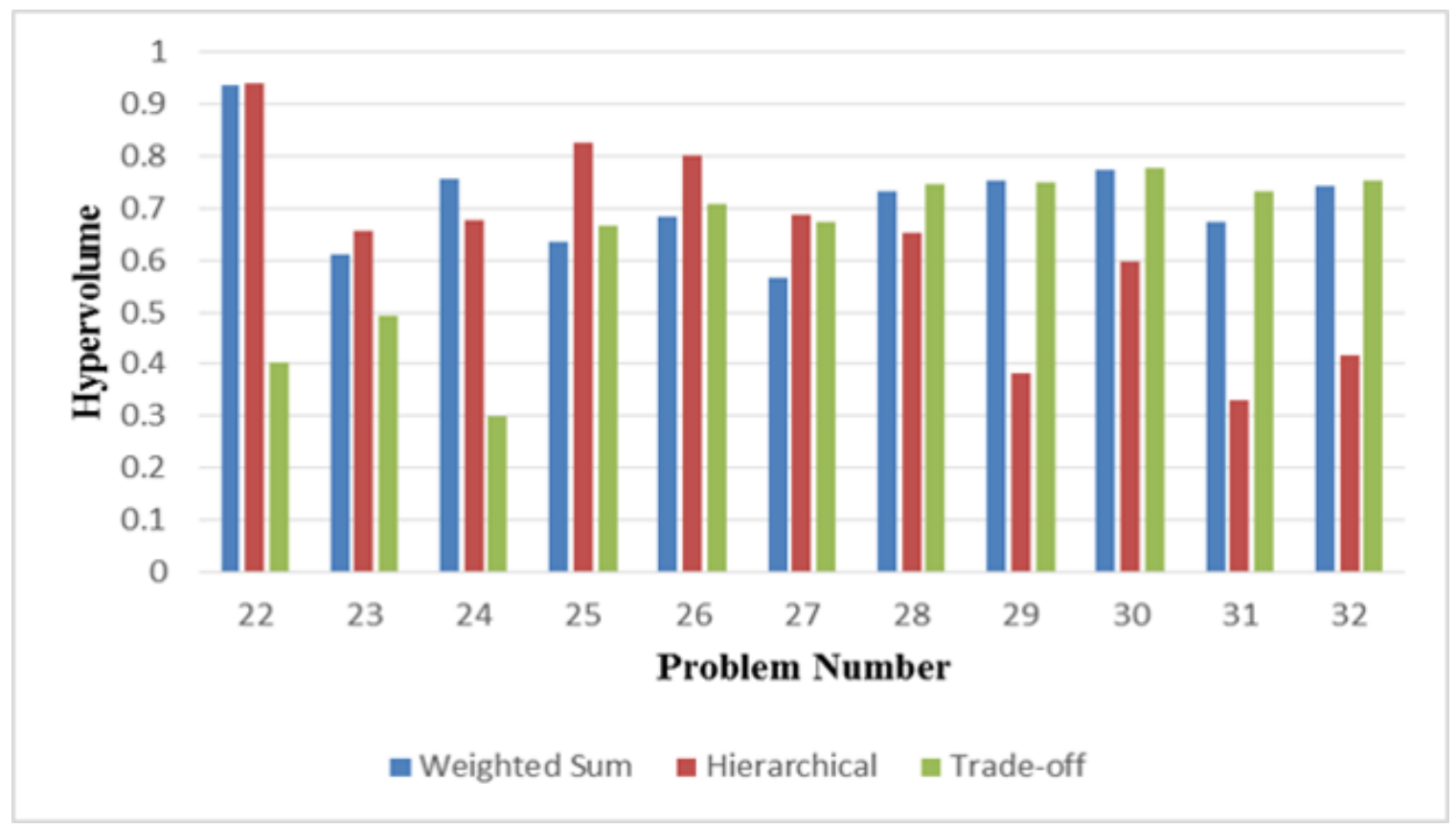

Figure 4.14: Average HV indicator for large scale problems

\section{The Hypervolume Indicator}

Figure 4.14 portrays the average HV for large scale FPP for the three methods. The highest HV of 0.9396 was achieved by the hierarchical method, whereas the tradeoff method gives the lowest $\mathrm{HV}$ of 0.2993 . It can be observed that the hierarchical and the trade-off method show an opposite pattern with the increase of problem size. For the first problem, the hierarchical method gives the best Pareto front but keeps on getting worse as the problem size increases and it is the opposite for the tradeoff method. Compared to the other two methods, the weighted sum gives a more consistent set of non-dominated solutions for all the problem sizes. Hence, it can be deduced that the weighted sum generates the best dominated space of the solutions in a Pareto front.

\subsubsection{Impact of the Number of Possible Placement Location}

In the previous two sections, we analyzed the results obtained for different number of edge device clusters with a fixed number of placement locations i.e., 5 and 10. In this section, we investigate the effect of the two different number of possible placement locations when the number of edge device clusters is set to $10,15,20,25,30,35,40$, 


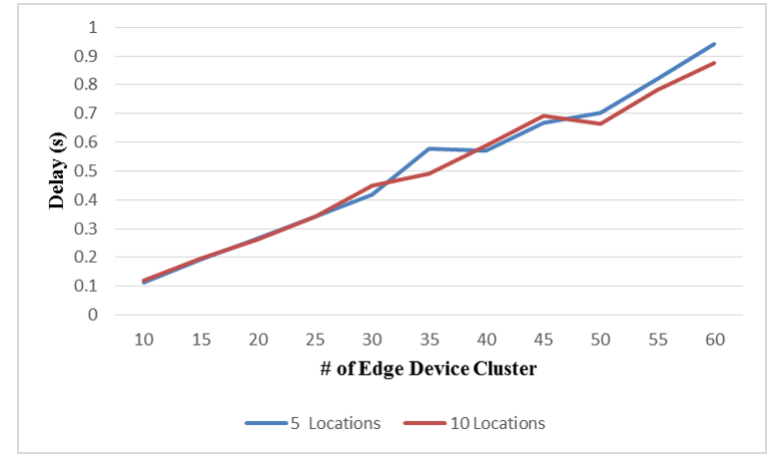

Figure 4.15: Delay comparison for different number of possible locations using the weighted sum method

$45,50,55$ and 60.

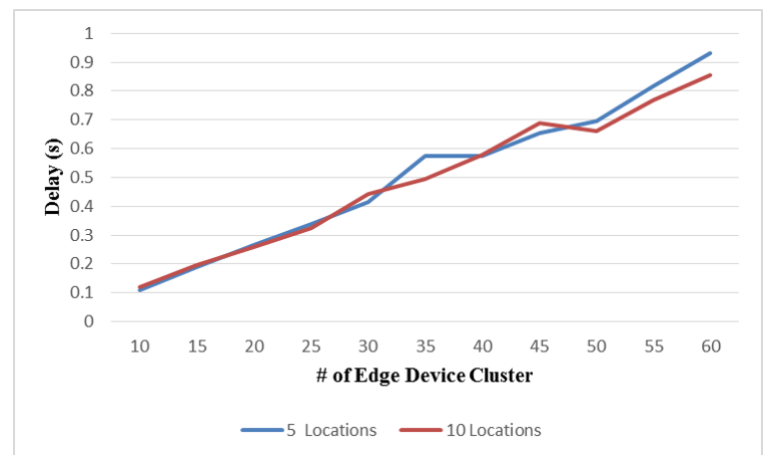

Figure 4.16: Delay comparison for different number of possible locations using the hierarchical method

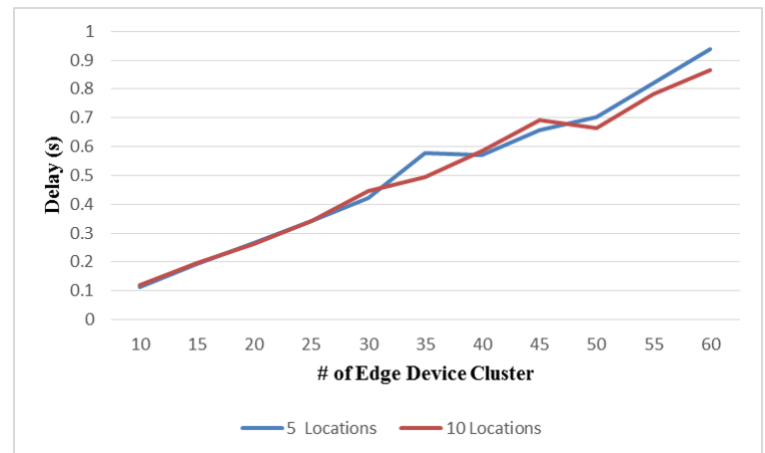

Figure 4.17: Delay comparison for different number of possible locations using the trade-off method

Figures 4.15 to 4.23 represent the results for each set of edge device clusters 


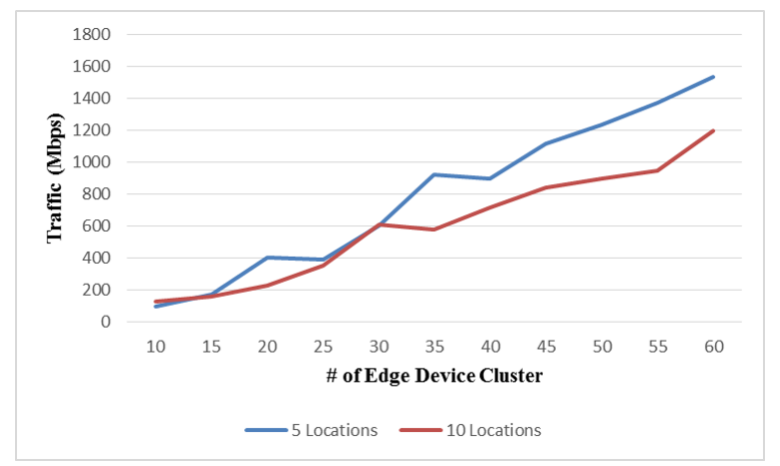

Figure 4.18: Traffic comparison for different number of possible locations using the weighted sum method

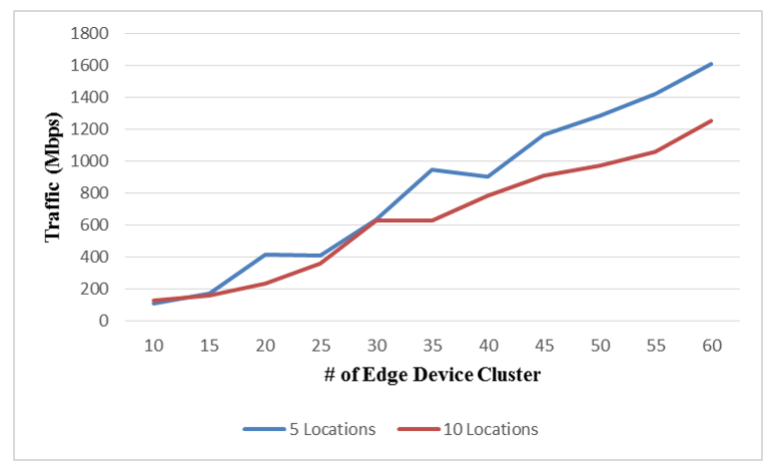

Figure 4.19: Traffic comparison for different number of possible locations using the hierarchical method

grouped by the number of maximum possible placement locations for the three methods respectively. From the figures, it can be seen that as the number of edge device clusters increases, the delay gap for the two different number of placement location is also increasing for all the three methods. To be exact, the delay is higher when only 5 possible placement locations are used. As more possible placement locations are added and for the same number of edge device clusters, more fog nodes can be installed in the network and better locations can be selected resulting in a decrease of the total network delay. Moreover, as the number of possible placement location is increasing, the budget is also increasing. In terms of the average traffic, a similar pattern can be observed. With the availability of more fog locations, less traffic is 


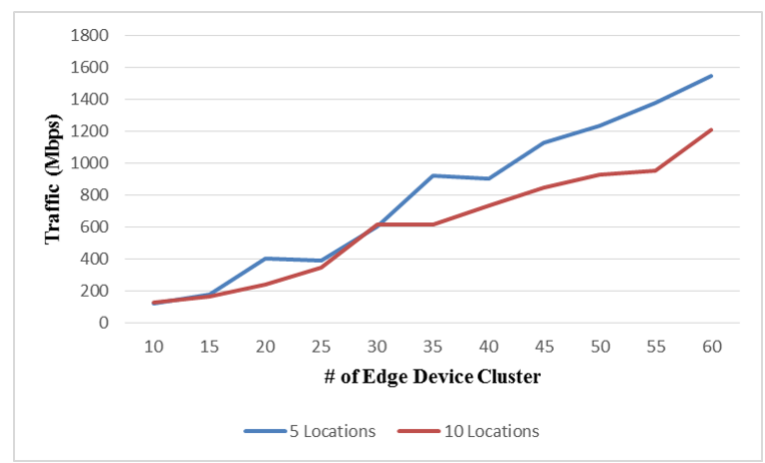

Figure 4.20: Traffic comparison for different number of possible locations using the trade-off method

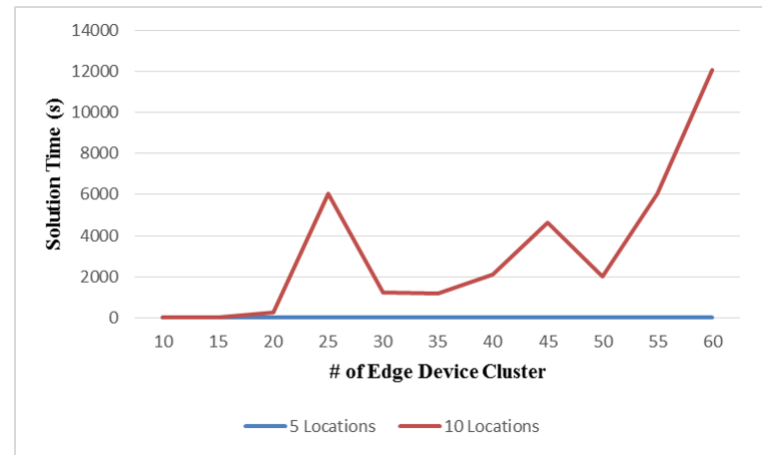

Figure 4.21: Solution time comparison for different number of possible locations using the weighted sum method

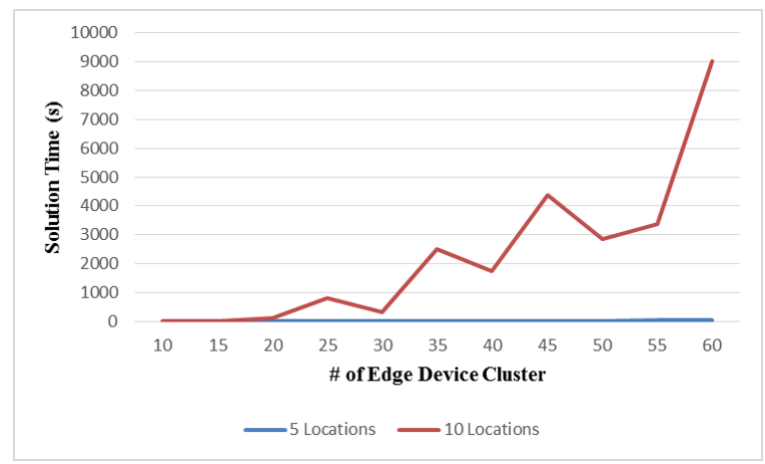

Figure 4.22: Solution time comparison for different number of possible locations using the hierarchical method 


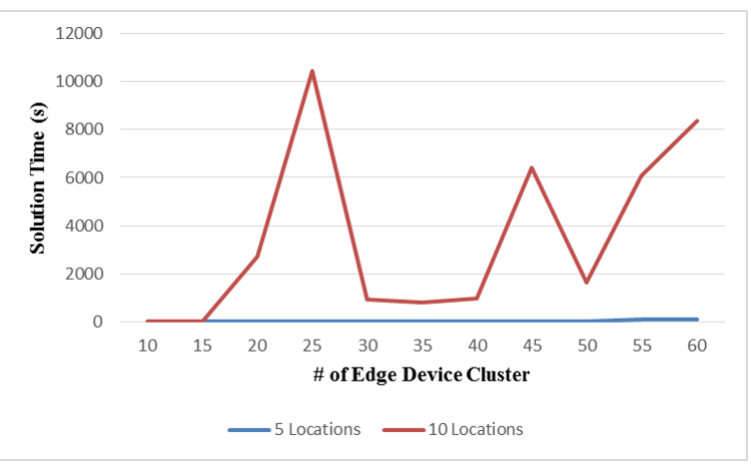

Figure 4.23: Solution time comparison for different number of possible locations using the trade-off method

sent to the cloud as most of the requests are processed at the fog layer.

Unlike the objective functions, the solution time plotted in figures 4.21 to 4.23 show more randomness. Moreover, a larger gap in the solution time can be observed for all the three methods. For example, the solution time for 60 edge device clusters and 5 possible placement locations is 24 seconds for the weighted sum, 35 seconds for the hierarchical and 73 seconds for the trade-off method. Whereas, for the same number of edge device cluster and 10 maximum placement locations, the solution time is 12054 seconds for the weighted sum, 9009 seconds for the hierarchical and 8370 seconds for the trade-off method. The larger gap is because the FPP is an integer linear programming problem which is NP-Hard 80].

\subsection{Chapter Summary}

Planning a fog network is a complex problem. Some of the difficult aspects of FPP include the finding of information about the input parameters, fog node features, finding the location to deploy fog nodes, the assignment of the edge device clusters, etc. In this chapter, we identified the features and characteristics of the proposed FPP based on the optimization results for different topologies. The results for the three multiobjective optimization methods were obtained in terms of total delay in the network, traffic in the cloud and solution time. The detailed example returned exactly the same result as the manually derived, helping in understanding the model.

To evaluate the FPP model, two different sets of problem sizes were generated. 21 
different problem sizes were solved for the small scale problems and 11 different problem sizes were solved for the large scale problems. With the increase of problem size, delay, traffic and solution time also increases. The objective functions had a linear pattern in the increment, whereas the solution time increased in a non linear form. This illustrates that the FPP is a NP-hard problem and further increase in problem size will increase the solution time exponentially. Furthermore, the three methods were compared, in terms of the objective functions for all the problems. For most of the problem sizes, the hierarchical method was able to return good solutions for the delay in the network at the expense of traffic. This makes the hierarchical method the best method for a delay sensitive network. Whereas, the weighted sum method achieved the best compromised result among the three multiobjective optimization methods while considering the trade-off between the objective functions which should be used for delay-traffic aware networks.

The performance of the methods used for the model was also analyzed showing that the multiobjective methods are able to give similar results compared to the single objective optimization. Moreover, the HV value indicates that the weighted sum and trade-off methods are able to generate satisfactory Pareto fronts. Finally, we compared the results for two different numbers of possible fog node placement locations. It was seen that with the same number of edge device cluster, if we increase the maximum possible placement locations, the delay in the network and the traffic in the cloud decreases but the solution time increases significantly.

The next chapter summarizes the thesis and describes the different improvements that can be done as future work. 


\section{Chapter 5}

\section{Conclusions and Future Work}

Since, fog networks are designed to be closer to the edge devices, it is very important to develop efficient network planning tools for the best service. This thesis studies the planning and design of a brand new fog networks by simultaneously finding the optimal location, number, capacity and the type of the fog nodes, as well as the interconnection between the fog nodes and the cloud. The optimization model minimizes the network delay and the traffic in the cloud which are two of the most important parameters when dealing with the fog networks. To obtain the optimal solutions, the memory, vCPU and the link speed of the edge devices were considered. The FPP is an integer linear problem that ensures the minimization of the objective functions with respect to uniqueness, capacity, assignment, budget and integrality constraints.

Before solving the planning model, different sizes of problems were generated with different edge device input parameters and randomized locations for edge device clusters and the possible fog node placements. A detailed example was solved to better understand the planning model. As the FPP contains two objective functions, three multiobjective methods were used to obtain optimal solutions. More precisely, the weighted sum, the hierarchical and the trade-off methods were used in solving the FPP and a fuzzy-based mechanism was used to obtain the best compromised results. Some general trends can be observed for the delay in the network, the traffic in the cloud and the solution time for the different topologies. As the input size increases, the objective functions and the solution time for each multiobjective optimization

method also increases. However, for the same number of edge device clusters and a higher number of possible fog node placement locations, the objective functions decrease but the solution time increases. For example, the planning problem with 50 
edge device clusters and 5 possible placement locations results in a minimum delay of 0.694 seconds and a minimum traffic of $1231 \mathrm{Mbps}$ with a lowest solution time of 7 seconds. Whereas, for the same number of edge device clusters and 10 possible fog node placement locations the optimal solution for delay is 0.66 seconds and the traffic is 897 Mbps with a lowest solution time of 897 seconds.

In term of the methods, the weighted sum method was able to return the best trade-off results for the delay and the traffic. The hierarchical method was able to achieve better delay but the traffic was worse compared to the other two methods. For small scale problems, the trade-off method generated the best set of non-dominated solutions. It shows that, for small scale problems, the trade-off method can be used for situations were the decision maker can make their own choice of best result from the set of non-dominated solutions, instead of using the fuzzy-based approach. The weighted sum method generated the best Pareto optimal set for the large scale problems.

\section{$5.1 \quad$ Future Works}

To the extent of our knowledge, research in planning and design of fog networks is a very new topic. Hence, the work in this study can be extended to many possible directions. Some of them are as follows:

- We have restricted our delay equation to transmission, propagation and processing delays. For a better approximation of the end-to-end delay, the addition of queuing delay could be a major improvement. For the FPP, the budget is limited to a fixed percentage of the maximum cost, in the future the model can be analyzed by varying the budget percentage.

- One of the problems with the weighted sum method is that it is impossible to obtain points on non-convex region of the Pareto front in the criterion space 81. The exponential weighted criterion method can be used to obtain the points on the non-convex region of the Pareto optimal set 82 . The hierarchical and the trade-off methods are modified according to a delay sensitive network, but sometimes it is hard for the decision makers to articulate a preference. In that case, different global criterion methods can be used to obtain a better trade-off between the objective functions. 
- The solution time for the exact algorithm is very high. To decrease the solution time, we can run many problems and test different parameter settings in CPLEX for each problem. As the FPP is a NP-hard problem and the solution time increases exponentially with the increase of input size, obtaining the best parameters for the solver will provide a negligible improvement. A viable improvement can be the use of multiobjective heuristic algorithms like Strength Pareto Evolutionary Algorithm (SPEA) or Non-dominated Sorting Genetic Algorithm II (NSGA-II). Although the solutions obtained will not be optimal, a significant improvement can be achieved in terms of solution time.

- After deploying the fog network, the next step is to manage the resource and the workload. In a dynamic network where the edge device location and density is changing, efficient algorithms need to be developed to assign the edge devices to appropriate fog nodes. The SDN architecture can be used for enhancement of performance and betterment of traffic and fog node management.

- Another future direction can be the expansion of fog networks. Once the fog network is planed and implemented, over time, more edge device clusters may be added to the network. The proposed model can be extended to an expansion model, where new fog nodes can be added (or replaced) without changing the whole network infrastructure. 


\section{List of References}

[1] "Micro-datacentre: What it problems it solves and what workload systems suit it." http://www.computerweekly.com/feature/ Micro-datacentre-What-IT-problems-it-solves-and-what-workload-systems-is-it-b (Accessed on 12/22/2017).

[2] F. Bonomi, R. Milito, J. Zhu, and S. Addepalli, "Fog computing and its role in the internet of things," in Proceedings of the First Edition of the MCC Workshop on Mobile Cloud Computing, MCC '12, (New York, NY, USA), pp. 13-16, ACM, 2012.

[3] S. Liu and M. St-Hilaire, "Cooperative algorithm for the global planning problem of umts networks," in IEEE Global Telecommunications Conference GLOBECOM, pp. 1-5, Dec 2010.

[4] S. Z. Ali and R. J. Read, "Design and performance evaluation of an optimization methodology for optimal solution of cellular layout design problems," in Signals, Circuits and Systems. International Symposium on SCS, vol. 2, pp. 521-524, 2003.

[5] P. Ngatchou, A. Zarei, and A. El-Sharkawi, "Pareto multi objective optimization," in Proceedings of the 13th International Conference on, Intelligent Systems Application to Power Systems, pp. 84-91, Nov 2005.

[6] C. Zhu, L. Xu, and E. D. Goodman, "Generalization of pareto-optimality for many-objective evolutionary optimization," IEEE Transactions on Evolutionary Computation, vol. 20, pp. 299-315, Apr 2016.

[7] Q. Zhang, L. Cheng, and R. Boutaba, "Cloud computing: state-of-the-art and research challenges," Journal of Internet Services and Applications, vol. 1, pp. 718, May 2010.

[8] B. Sotomayor, R. S. Montero, I. M. Llorente, and I. Foster, "Virtual infrastructure management in private and hybrid clouds," IEEE Internet Computing, vol. 13, pp. 14-22, Sept 2009.

[9] L. M. Vaquero, L. Rodero-Merino, J. Caceres, and M. Lindner, "A break in the clouds: Towards a cloud definition," SIGCOMM Comput. Commun. Rev., vol. 39, pp. 50-55, Dec. 2008. 
[10] I. Foster and C. Kesselman, eds., The Grid: Blueprint for a New Computing Infrastructure. San Francisco, CA, USA: Morgan Kaufmann Publishers Inc., 1999.

[11] L. K. Arya and A. Verma, "Workflow scheduling algorithms in cloud environment - a survey," in Recent Advances in Engineering and Computational Sciences (RAECS), pp. 1-4, Mar 2014.

[12] I. Management Association, Grid and Cloud Computing: Concepts, Methodologies, Tools and Applications: Concepts, Methodologies, Tools and Applications. Premier reference source, Information Science Reference, 2012.

[13] M. R. Nami and K. Bertels, "A survey of autonomic computing systems," in Autonomic and Autonomous Systems. Third International Conference on ICAS, pp. 26-26, Jun 2007.

[14] R. Buyya, C. S. Yeo, and S. Venugopal, "Market-oriented cloud computing: Vision, hype, and reality for delivering it services as computing utilities," in High Performance Computing and Communications.10th IEEE International Conference on $H P C C$, pp. 5-13, IEEE, 2008.

[15] T. Dillon, C. Wu, and E. Chang, "Cloud computing: Issues and challenges," in 24th IEEE International Conference on Advanced Information Networking and Applications, pp. 27-33, Apr 2010.

[16] Y. Jadeja and K. Modi, "Cloud computing - concepts, architecture and challenges," in International Conference on Computing, Electronics and Electrical Technologies (ICCEET), pp. 877-880, Mar 2012.

[17] S. Ramgovind, M. M. Eloff, and E. Smith, "The management of security in cloud computing," in Information Security for South Africa, pp. 1-7, Aug 2010.

[18] O. Biran, A. Corradi, M. Fanelli, L. Foschini, A. Nus, D. Raz, and E. Silvera, "A stable network-aware vm placement for cloud systems," in 12th IEEE/ACM International Symposium on Cluster, Cloud and Grid Computing (ccgrid 2012), pp. 498-506, May 2012.

[19] I. Hwang and M. Pedram, "Hierarchical virtual machine consolidation in a cloud computing system," in IEEE Sixth International Conference on Cloud Computing, pp. 196-203, Jun 2013.

[20] B. Kantarci, L. Foschini, A. Corradi, and H. T. Mouftah, "Inter-and-intra data center vm-placement for energy-efficient large-scale cloud systems," in IEEE Globecom Workshops, pp. 708-713, Dec 2012.

[21] W. Fang, X. Liang, S. Li, L. Chiaraviglio, and N. Xiong, "Vmplanner: Optimizing virtual machine placement and traffic flow routing to reduce network power costs in cloud data centers," Computer Networks, vol. 57, no. 1, pp. $179-196$, 2013.

[22] J. Dong, X. Jin, H. Wang, Y. Li, P. Zhang, and S. Cheng, "Energy-saving virtual machine placement in cloud data centers," in 13th IEEE/ACM International Symposium on Cluster, Cloud, and Grid Computing, pp. 618-624, May 2013. 
[23] T. C. Ferreto, M. A. Netto, R. N. Calheiros, and C. A. D. Rose, "Server consolidation with migration control for virtualized data centers," Future Generation Computer Systems, vol. 27, no. 8, pp. 1027 - 1034, 2011.

[24] N. Kord and H. Haghighi, "An energy-efficient approach for virtual machine placement in cloud based data centers," in The 5th Conference on Information and Knowledge Technology, pp. 44-49, May 2013.

[25] P. Hofmann and D. Woods, "Cloud computing: The limits of public clouds for business applications," IEEE Internet Computing, vol. 14, no. 6, pp. 90-93, 2010.

[26] G. Pardo-Castellote, "Omg data-distribution service: architectural overview," in 23rd International Conference on Distributed Computing Systems Workshops. Proceedings., pp. 200-206, May 2003.

[27] Z. Shelby, K. Hartke, and C. Bormann, "The constrained application protocol (coap)," 2014.

[28] M. Singh, M. A. Rajan, V. L. Shivraj, and P. Balamuralidhar, "Secure mqtt for internet of things (iot)," in Fifth International Conference on Communication Systems and Network Technologies, pp. 746-751, Apr 2015.

[29] S. Vinoski, "Advanced message queuing protocol," IEEE Internet Computing, vol. 10, pp. 87-89, Nov 2006.

[30] D. Costa, E. Mingozzi, G. Tanganelli, and C. Vallati, "An alljoyn to coap bridge," in IEEE 3rd World Forum on Internet of Things (WF-IoT), pp. 395-400, Dec 2016.

[31] K. Hong, D. Lillethun, U. Ramachandran, B. Ottenwälder, and B. Koldehofe, "Mobile fog: A programming model for large-scale applications on the internet of things," in Proceedings of the Second ACM SIGCOMM Workshop on Mobile Cloud Computing, MCC '13, (New York, NY, USA), pp. 15-20, ACM, 2013.

[32] I. Stojmenovic, "Fog computing: A cloud to the ground support for smart things and machine-to-machine networks," in Australasian Telecommunication Networks and Applications Conference (ATNAC), pp. 117-122, Nov 2014.

[33] V. Stantchev, A. Barnawi, S. Ghulam Muhammad, J. Schubert, and G. Tamm, "Smart items, fog and cloud computing as enablers of servitization in healthcare," vol. 185, pp. 121 - 128, 022015.

[34] Y. Cao, S. Chen, P. Hou, and D. Brown, "Fast: A fog computing assisted distributed analytics system to monitor fall for stroke mitigation," in IEEE International Conference on Networking, Architecture and Storage (NAS), pp. 2-11, Aug 2015.

[35] T. N. Gia, M. Jiang, A. M. Rahmani, T. Westerlund, P. Liljeberg, and H. Tenhunen, "Fog computing in healthcare internet of things: A case study on ecg feature extraction," in IEEE International Conference on Computer and Information Technology; Ubiquitous Computing and Communications; Dependable, Autonomic and Secure Computing; Pervasive Intelligence and Computing, pp. 356363 , Oct 2015. 
[36] M. Aazam and E. N. Huh, "Fog computing and smart gateway based communication for cloud of things," in International Conference on Future Internet of Things and Cloud, pp. 464-470, Aug 2014.

[37] J. Zhu, D. S. Chan, M. S. Prabhu, P. Natarajan, H. Hu, and F. Bonomi, "Improving web sites performance using edge servers in fog computing architecture," in IEEE Seventh International Symposium on Service-Oriented System Engineering, pp. 320-323, Mar 2013.

[38] "Openfog consortium -." https://www.openfogconsortium.org/. (Accessed on $11 / 09 / 2017)$.

[39] S. Kosta, A. Aucinas, P. Hui, R. Mortier, and X. Zhang, "Thinkair: Dynamic resource allocation and parallel execution in the cloud for mobile code offloading," in 2012 Proceedings IEEE INFOCOM, pp. 945-953, Mar 2012.

[40] B.-G. Chun, S. Ihm, P. Maniatis, M. Naik, and A. Patti, "Clonecloud: Elastic execution between mobile device and cloud," in Proceedings of the Sixth Conference on Computer Systems, EuroSys '11, (New York, NY, USA), pp. 301-314, ACM, 2011.

[41] G. Orsini, D. Bade, and W. Lamersdorf, "Computing at the mobile edge: Designing elastic android applications for computation offloading," in 8th IFIP Wireless and Mobile Networking Conference (WMNC), pp. 112-119, Oct 2015.

[42] F. Jalali, K. Hinton, R. Ayre, T. Alpcan, and R. S. Tucker, "Fog computing may help to save energy in cloud computing," IEEE Journal on Selected Areas in Communications, vol. 34, pp. 1728-1739, May 2016.

[43] R. Deng, R. Lu, C. Lai, and T. H. Luan, "Towards power consumption-delay tradeoff by workload allocation in cloud-fog computing.," in $I C C$, pp. 3909-3914, IEEE, 2015.

[44] I. Stojmenovic, S. Wen, X. Huang, and H. Luan, "An overview of fog computing and its security issues," Concurrency and Computation: Practice and Experience, vol. 28, no. 10 , pp. 2991-3005, 2016. cpe.3485.

[45] Y. W. Law, M. Palaniswami, G. Kounga, and A. Lo, "Wake: Key management scheme for wide-area measurement systems in smart grid," IEEE Communications Magazine, vol. 51, pp. 34-41, Jan 2013.

[46] C. Marforio and K. K. S. C. Nikolaos Karapanos, Claudio Soriente, "Smartphones as practical and secure location verification tokens for payments," in 21st Annual Network and Distributed System Security Symposium, NDSS 2014, San Diego, California, USA, February 23-26, 2014.

[47] C. Dsouza, G. J. Ahn, and M. Taguinod, "Policy-driven security management for fog computing: Preliminary framework and a case study," in Proceedings of the IEEE 15th International Conference on Information Reuse and Integration (IEEE IRI 2014), pp. 16-23, Aug 2014. 
[48] D. Zeng, L. Gu, S. Guo, Z. Cheng, and S. Yu, "Joint optimization of task scheduling and image placement in fog computing supported software-defined embedded system," IEEE Transactions on Computers, vol. 65, pp. 3702-3712, Dec 2016.

[49] M. Aazam and E. N. Huh, "Fog computing micro datacenter based dynamic resource estimation and pricing model for iot," in IEEE 29th International Conference on Advanced Information Networking and Applications, pp. 687-694, Mar 2015.

[50] M. Malensek, S. L. Pallickara, and S. Pallickara, "Hermes: Federating fog and cloud domains to support query evaluations in continuous sensing environments," IEEE Cloud Computing, vol. 4, pp. 54-62, Mar 2017.

[51] R. Schoenen and H. Yanikomeroglu, "User-in-the-loop: spatial and temporal demand shaping for sustainable wireless networks," IEEE Communications Magazine, vol. 52, pp. 196-203, Feb 2014.

[52] D. Sabella, A. Vaillant, P. Kuure, U. Rauschenbach, and F. Giust, "Mobileedge computing architecture: The role of mec in the internet of things," IEEE Consumer Electronics Magazine, vol. 5, pp. 84-91, Oct 2016.

[53] T. Piliouras, Network design: management and technical perspectives. Auerbach, 2005.

[54] H. D. Sherali, C. M. Pendyala, and T. S. Rappaport, "Optimal location of transmitters for micro-cellular radio communication system design," IEEE Journal on Selected Areas in Communications, vol. 14, pp. 662-673, May 1996.

[55] H. R. Anderson and J. P. McGeehan, "Optimizing microcell base station locations using simulated annealing techniques," in Proceedings of IEEE Vehicular Technology Conference (VTC), pp. 858-862 vol.2, Jun 1994.

[56] M. St-Hilaire, S. Chamberland, and S. Pierre, "A tabu search algorithm for the global planning problem of third generation mobile networks," Computers \&6 Electrical Engineering, vol. 34, no. 6, pp. 470 - 487, 2008.

[57] M. R. Pasandideh and M. St-Hilaire, "Automatic planning of 3g umts all-ip release 4 networks with realistic traffic," Comput. Oper. Res., vol. 40, pp. 19912003, Aug. 2013.

[58] B. Heller, R. Sherwood, and N. McKeown, "The controller placement problem," in Proceedings of the First Workshop on Hot Topics in Software Defined Networks, HotSDN '12, (New York, NY, USA), pp. 7-12, ACM, 2012.

[59] A. Sallahi and M. St-Hilaire, "Optimal model for the controller placement problem in software defined networks," IEEE Communications Letters, vol. 19, pp. 30-33, Jan 2015.

[60] "Ubc radio science lab." http://rsl.ece.ubc.ca/planning.html. (Accessed on $11 / 10 / 2017)$. 
[61] L. Cen, K. F. Poon, Z. L. Yu, and A. Ouali, "Automatic planning of gpon/ftth networks based on lagrangian heuristic optimization," in IEEE International Conference on Industrial Engineering and Engineering Management, pp. 335339, Dec 2013.

[62] B. Addis, D. Belabed, M. Bouet, and S. Secci, "Virtual network functions placement and routing optimization," in IEEE 4th International Conference on Cloud Networking (CloudNet), pp. 171-177, Oct 2015.

[63] H. Moens and F. D. Turck, "Vnf-p: A model for efficient placement of virtualized network functions," in 10th International Conference on Network and Service Management (CNSM) and Workshop, pp. 418-423, Nov 2014.

[64] C. Pham, N. H. Tran, S. Ren, W. Saad, and C. S. Hong, "Traffic-aware and energy-efficient vnf placement for service chaining: Joint sampling and matching approach," IEEE Transactions on Services Computing, vol. PP, no. 99, pp. 1-1, 2017.

[65] B. Blanco, I. Taboada, J. O. Fajardo, and F. Liberal, "A robust optimization based energy-aware virtual network function placement proposal for small cell $5 \mathrm{~g}$ networks with mobile edge computing capabilities," Mobile Information Systems, vol. 2017, no. 2603410, p. 14, 2017.

[66] M. Cvijetic and I. Djordjevic, Advanced Optical Communication Systems and Networks. Artech House applied photonics series, Artech House, 2013.

[67] "Alcatel-lucent sets new world record broadband speed of 10 gbps for transmission of data over traditional copper telephone lines - nokia networks." https://networks.nokia.com/press/2014/ alcatel-lucent-sets-new-world-record-broadband-speed-10-gbps-transmission-dat (Accessed on 12/20/2017).

[68] I. Cisco Systems, "Design best practices for latency optimization," tech. rep., Cisco Systems, Inc., CA, USA, 2007.

[69] W. Jakob and C. Blume, "Pareto optimization or cascaded weighted sum: A comparison of concepts," vol. 7, pp. 166-185, 032014.

[70] G. Anandalingam and T. L. Friesz, "Hierarchical optimization: An introduction," Annals of Operations Research, vol. 34, pp. 1-11, Dec 1992.

[71] V. Chankong and Y. Haimes, Multiobjective decision making: theory and methodology. North-Holland series in system science and engineering, North Holland, 1983.

[72] G. Mavrotas, "Effective implementation of the $\epsilon$-constraint method in multiobjective mathematical programming problems," Appl. Math. Comput., vol. 213, pp. 455-465, Jul 2009.

[73] B. Panigrahi, V. Pandi, R. Sharma, S. Das, and S. Das, "Multiobjective bacteria foraging algorithm for electrical load dispatch problem," vol. 52, pp. 1334-1342, 022011. 
[74] E. Zitzler and L. Thiele, "Multiobjective optimization using evolutionary algorithms - a comparative case study," in Proceedings of the 5th International Conference on Parallel Problem Solving from Nature, PPSN V, (London, UK, UK), pp. 292-304, Springer-Verlag, 1998.

[75] A. Custdio, M. Emmerich, and J. Madeira, "A robust optimization based energyaware virtual network function placement proposal for small cell $5 \mathrm{~g}$ networks with mobile edge computing capabilities," Computational Technology Reviews, vol. 5, pp. 1-30, Apr 2012.

[76] Ibm, IBM ILOG CPLEX Optimization Studio CPLEX User's Manual, 2011.

[77] I. A. Kateeb, K. F. AlOtaib, L. Burton, and M. S. Peluso, "Cooperative algorithm for the global planning problem of umts networks," in Proceedings of the 2013 American Society for Engineering Education Pacific Southwest Conference, pp. 452-467, 2013.

[78] "Managed dedicated server hosting — rackspace hosting experts." https://www . rackspace.com/dedicated-servers. (Accessed on 08/12/2017).

[79] J. Clausen, "Branch and bound algorithms - principles and examples," 1999.

[80] C. H. Papadimitriou, "On the complexity of integer programming," J. $A C M$, vol. 28, pp. 765-768, Oct. 1981.

[81] I. Das and J. Dennis, "A closer look at drawbacks of minimizing weighted sums of objectives for pareto set generation in multicriteria optimization problems," vol. 14, pp. 63-69, 011997.

[82] T. W. ATHAN and P. Y. PAPALAMBROS, "A note on weighted criteria methods for compromise solutions in multi-objective optimization," Engineering Optimization, vol. 27, no. 2, pp. 155-176, 1996. 


\section{Appendix A}

\section{Results for Small Scale Problem}

\section{A.1 Instance 1}

Table A.1: Result obtained from the solver for small scale problem: Instance 1

\begin{tabular}{|c|c|c|c|c|c|c|c|c|c|}
\hline & \multicolumn{3}{|c|}{ Weighted Sum } & \multicolumn{3}{c|}{ Hierarchical } & \multicolumn{3}{c|}{ Trade-off } \\
\hline \hline Problem & $\begin{array}{c}\text { Delay } \\
(\mathrm{s})\end{array}$ & $\begin{array}{c}\text { Traffic } \\
(\text { Mbps }\end{array}$ & $\begin{array}{c}\text { Solution Time } \\
(\mathrm{s})\end{array}$ & $\begin{array}{c}\text { Delay } \\
(\mathrm{s})\end{array}$ & $\begin{array}{c}\text { Traffic } \\
(\mathrm{Mbps})\end{array}$ & $\begin{array}{c}\text { Solution Time } \\
(\mathrm{s})\end{array}$ & $\begin{array}{c}\text { Delay } \\
(\mathrm{s})\end{array}$ & $\begin{array}{c}\text { Traffic } \\
(\mathrm{Mbps})\end{array}$ & $\begin{array}{c}\text { Solution Time } \\
(\mathrm{s})\end{array}$ \\
\hline \hline 1 & 0.104 & 67 & 1 & 0.098 & 110 & 3 & 0.104 & 78 & 2 \\
\hline 2 & 0.177 & 116 & 2 & 0.177 & 116 & 3 & 0.177 & 116 & 2 \\
\hline 3 & 0.277 & 448 & 2 & 0.27 & 474 & 4 & 0.277 & 448 & 2 \\
\hline 4 & 0.354 & 554 & 3 & 0.348 & 595 & 7 & 0.354 & 554 & 6 \\
\hline 5 & 0.387 & 631 & 3 & 0.387 & 631 & 8 & 0.387 & 631 & 3 \\
\hline 6 & 0.582 & 815 & 3 & 0.576 & 857 & 10 & 0.582 & 815 & 3 \\
\hline 7 & 0.651 & 1130 & 16 & 0.651 & 1130 & 24 & 0.651 & 1130 & 31 \\
\hline 8 & 0.664 & 953 & 2 & 0.62 & 1064 & 18 & 0.626 & 1011 & 11 \\
\hline 9 & 0.671 & 1246 & 15 & 0.665 & 1319 & 20 & 0.671 & 1256 & 56 \\
\hline 10 & 0.792 & 1224 & 119 & 0.786 & 1292 & 45 & 0.792 & 1224 & 265 \\
\hline 11 & 0.996 & 1576 & 14 & 0.984 & 1643 & 33 & 0.99 & 1607 & 27 \\
\hline 12 & 1.118 & 1853 & 10 & 1.106 & 1917 & 36 & 1.119 & 1850 & 44 \\
\hline 13 & 1.06 & 1825 & 8 & 1.054 & 1914 & 37 & 1.06 & 1825 & 110 \\
\hline 14 & 1.113 & 1879 & 10 & 1.107 & 1908 & 94 & 1.113 & 1879 & 232 \\
\hline 15 & 1.305 & 2099 & 11 & 1.305 & 2102 & 46 & 1.304 & 2102 & 106 \\
\hline 16 & 1.265 & 2269 & 8 & 1.254 & 2395 & 65 & 1.265 & 2298 & 76 \\
\hline 17 & 1.479 & 2628 & 2801 & 1.479 & 2628 & 66 & 1.479 & 2630 & 693 \\
\hline 18 & 1.401 & 2561 & 279 & 1.389 & 2643 & 83 & 1.401 & 2561 & 1375 \\
\hline 19 & 1.538 & 2732 & 29 & 1.539 & 2732 & 74 & 1.538 & 2740 & 44 \\
\hline 20 & 2.42 & 4504 & 83 & 2.415 & 4533 & 186 & 2.426 & 4481 & 130 \\
\hline 21 & 3.13 & 5356 & 1548 & 3.124 & 5385 & 1107 & 3.135 & 5348 & 9019 \\
\hline
\end{tabular}




\section{A.2 Instance 2}

Table A.2: Result obtained from the solver for small scale problem: Instance 2

\begin{tabular}{|c|c|c|c|c|c|c|c|c|c|}
\hline & \multicolumn{3}{|c|}{ Weighted Sum } & \multicolumn{3}{|c|}{ Hierarchical } & \multicolumn{3}{|c|}{ Trade-off } \\
\hline Problem & $\begin{array}{c}\text { Delay } \\
(\mathrm{s})\end{array}$ & $\begin{array}{l}\text { Traffic } \\
\text { (Mbps) }\end{array}$ & $\begin{array}{c}\text { Solution Time } \\
(\mathrm{s})\end{array}$ & $\begin{array}{c}\text { Delay } \\
(\mathrm{s})\end{array}$ & $\begin{array}{l}\text { Traffic } \\
\text { (Mbps) }\end{array}$ & $\begin{array}{c}\text { Solution Time } \\
(\mathrm{s})\end{array}$ & $\begin{array}{c}\text { Delay } \\
(\mathrm{s})\end{array}$ & $\begin{array}{l}\text { Traffic } \\
\text { (Mbps) }\end{array}$ & $\begin{array}{c}\text { Solution Time } \\
(\mathrm{s})\end{array}$ \\
\hline 1 & 0.068 & 11 & 1 & 0.068 & 11 & 2 & 0.068 & 101 & 2 \\
\hline 2 & 0.225 & 277 & 1 & 0.225 & 277 & 3 & 0.225 & 277 & 2 \\
\hline 3 & 0.269 & 420 & 2 & 0.269 & 420 & 4 & 0.269 & 420 & 2 \\
\hline 4 & 0.4 & 435 & 3 & 0.4 & 435 & 6 & 0.4 & 435 & 2 \\
\hline 5 & 0.444 & 491 & 3 & 0.444 & 491 & 8 & 0.45 & 469 & 5 \\
\hline 6 & 0.549 & 910 & 4 & 0.543 & 990 & 14 & 0.549 & 910 & 14 \\
\hline 7 & 0.634 & 937 & 3 & 0.629 & 961 & 12 & 0.64 & 925 & 5 \\
\hline 8 & 0.584 & 987 & 3 & 0.578 & 1061 & 21 & 0.584 & 987 & 46 \\
\hline 9 & 0.702 & 1247 & 5 & 0.695 & 1279 & 19 & 0.702 & 1248 & 9 \\
\hline 10 & 0.817 & 1302 & 8 & 0.811 & 1374 & 22 & 0.817 & 1302 & 27 \\
\hline 11 & 0.822 & 1312 & 14 & 0.816 & 1442 & 28 & 0.822 & 1309 & 164 \\
\hline 12 & 0.916 & 1460 & 134 & 0.909 & 1511 & 44 & 0.916 & 1460 & 75 \\
\hline 13 & 1.16 & 1995 & 91 & 1.153 & 2052 & 56 & 1.16 & 1995 & 178 \\
\hline 14 & 1.177 & 2050 & 5 & 1.158 & 2215 & 67 & 1.177 & 2050 & 48 \\
\hline 15 & 1.199 & 2212 & 24 & 1.192 & 2253 & 52 & 1.205 & 2189 & 2435 \\
\hline 16 & 1.315 & 2044 & 557 & 1.309 & 2087 & 69 & 1.315 & 2044 & 221 \\
\hline 17 & 1.277 & 1713 & 8 & 1.264 & 1778 & 57 & 1.276 & 1725 & 79 \\
\hline 18 & 1.629 & 3007 & 207 & 1.624 & 3034 & 69 & 1.629 & 3007 & 118 \\
\hline 19 & 1.55 & 3268 & 9 & 1.526 & 3340 & 74 & 1.543 & 3294 & 23 \\
\hline 20 & 2.384 & 3939 & 149 & 2.374 & 4013 & 186 & 2.384 & 3942 & 759 \\
\hline 21 & 3.318 & 5682 & 5484 & 3.311 & 5725 & 286 & 3.324 & 5665 & 1759 \\
\hline
\end{tabular}




\section{A.3 Instance 3}

Table A.3: Result obtained from the solver for small scale problem: Instance 3

\begin{tabular}{|c|c|c|c|c|c|c|c|c|c|}
\hline & \multicolumn{3}{|c|}{ Weighted Sum } & \multicolumn{3}{|c|}{ Hierarchical } & \multicolumn{3}{|c|}{ Trade-off } \\
\hline Problem & $\begin{array}{l}\text { Delay } \\
\text { (s) }\end{array}$ & $\begin{array}{l}\text { Traffic } \\
\text { (Mbps) }\end{array}$ & $\begin{array}{c}\text { Solution Time } \\
(\mathrm{s})\end{array}$ & $\begin{array}{c}\text { Delay } \\
(\mathrm{s})\end{array}$ & $\begin{array}{l}\text { Traffic } \\
\text { (Mbps) }\end{array}$ & $\begin{array}{c}\text { Solution Time } \\
(\mathrm{s})\end{array}$ & $\begin{array}{c}\text { Delay } \\
(\mathrm{s})\end{array}$ & $\begin{array}{l}\text { Traffic } \\
\text { (Mbps) }\end{array}$ & $\begin{array}{c}\text { Solution Time } \\
(\mathrm{s})\end{array}$ \\
\hline 1 & 0.167 & 255 & 2 & 0.167 & 255 & 2 & 0.167 & 255 & 1 \\
\hline 2 & 0.178 & 155 & 2 & 0.178 & 155 & 4 & 0.178 & 155 & 3 \\
\hline 3 & 0.287 & 352 & 3 & 0.287 & 352 & 5 & 0.287 & 352 & 2 \\
\hline 4 & 0.261 & 241 & 3 & 0.255 & 268 & 6 & 0.261 & 241 & 3 \\
\hline 5 & 0.44 & 644 & 2 & 0.433 & 697 & 8 & 0.44 & 644 & 4 \\
\hline 6 & 0.554 & 798 & 4 & 0.554 & 798 & 11 & 0.554 & 800 & 3 \\
\hline 7 & 0.531 & 613 & 4 & 0.518 & 676 & 13 & 0.524 & 646 & 7 \\
\hline 8 & 0.736 & 1306 & 6 & 0.736 & 1306 & 16 & 0.736 & 1306 & 12 \\
\hline 9 & 0.733 & 1175 & 3 & 0.726 & 1211 & 24 & 0.733 & 1175 & 10 \\
\hline 10 & 0.926 & 1633 & 4 & 0.921 & 1679 & 23 & 0.926 & 1633 & 10 \\
\hline 11 & 1.016 & 1660 & 4 & 1.003 & 1738 & 28 & 1.016 & 1660 & 11 \\
\hline 12 & 0.959 & 1450 & 9 & 0.952 & 1533 & 36 & 0.959 & 1433 & 36 \\
\hline 13 & 0.995 & 1608 & 3502 & 1.026 & 1515 & 36 & 0.994 & 1633 & 3152 \\
\hline 14 & 1.269 & 2031 & 472 & 1.269 & 2031 & 52 & 1.275 & 2013 & 99 \\
\hline 15 & 1.182 & 1889 & 25 & 1.175 & 1967 & 68 & 1.182 & 1889 & 492 \\
\hline 16 & 1.385 & 2715 & 16 & 1.385 & 2715 & 55 & 1.385 & 2715 & 1471 \\
\hline 17 & 0.905 & 2128 & 6 & 1.279 & 2188 & 62 & 1.284 & 2155 & 159 \\
\hline 18 & 0.996 & 2624 & 5 & 0.995 & 2660 & 68 & 0.994 & 2684 & 10 \\
\hline 19 & 1.592 & 2958 & 49 & 1.581 & 3037 & 75 & 1.592 & 2958 & 411 \\
\hline 20 & 2.354 & 3559 & 183 & 2.35 & 3572 & 178 & 2.354 & 3559 & 50 \\
\hline 21 & 3.257 & 6026 & 7094 & 3.252 & 6046 & 298 & 3.25 & 6046 & 3075 \\
\hline
\end{tabular}




\section{A.4 Instance 4}

Table A.4: Result obtained from the solver for small scale problem: Instance 4

\begin{tabular}{|c|c|c|c|c|c|c|c|c|c|}
\hline & \multicolumn{3}{|c|}{ Weighted Sum } & \multicolumn{3}{|c|}{ Hierarchical } & \multicolumn{3}{|c|}{ Trade-off } \\
\hline Problem & $\begin{array}{l}\text { Delay } \\
(\mathrm{s})\end{array}$ & $\begin{array}{l}\text { Traffic } \\
\text { (Mbps) }\end{array}$ & $\begin{array}{c}\text { Solution Time } \\
(\mathrm{s})\end{array}$ & $\begin{array}{l}\text { Delay } \\
(\mathrm{s})\end{array}$ & $\begin{array}{l}\text { Traffic } \\
\text { (Mbps) }\end{array}$ & $\begin{array}{l}\text { Solution Time } \\
\text { (s) }\end{array}$ & $\begin{array}{c}\text { Delay } \\
(\mathrm{s})\end{array}$ & $\begin{array}{c}\text { Traffic } \\
\text { (Mbps) }\end{array}$ & $\begin{array}{l}\text { Solution Time } \\
(\mathrm{s})\end{array}$ \\
\hline 1 & 0.111 & 56 & 1 & 0.111 & 64 & 2 & 0.111 & 56 & 1 \\
\hline 2 & 0.192 & 153 & 2 & 0.184 & 152 & 3 & 0.192 & 153 & 2 \\
\hline 3 & 0.232 & 384 & 4 & 0.232 & 420 & 5 & 0.232 & 384 & 3 \\
\hline 4 & 0.358 & 334 & 3 & 0.358 & 334 & 6 & 0.358 & 334 & 5 \\
\hline 5 & 0.405 & 648 & 3 & 0.393 & 719 & 8 & 0.405 & 651 & 3 \\
\hline 6 & 0.62 & 1152 & 2 & 0.62 & 1152 & 13 & 0.62 & 1152 & 3 \\
\hline 7 & 0.474 & 913 & 4 & 0.493 & 855 & 12 & 0.474 & 913 & 13 \\
\hline 8 & 0.691 & 1200 & 3 & 0.685 & 1240 & 18 & 0.691 & 1200 & 8 \\
\hline 9 & 0.697 & 1267 & 3 & 0.691 & 1321 & 20 & 0.703 & 1245 & 28 \\
\hline 10 & 0.751 & 1332 & 8 & 0.751 & 1332 & 26 & 0.751 & 1337 & 20 \\
\hline 11 & 0.929 & 1584 & 63 & 0.922 & 1612 & 51 & 0.928 & 1596 & 89 \\
\hline 12 & 1.004 & 1900 & 20 & 0.985 & 2011 & 34 & 0.997 & 1943 & 45 \\
\hline 13 & 1.053 & 1680 & 24 & 1.078 & 1646 & 38 & 1.053 & 1689 & 550 \\
\hline 14 & 1.119 & 1875 & 106 & 1.106 & 1966 & 57 & 1.119 & 1883 & 312 \\
\hline 15 & 1.154 & 1882 & 9 & 1.142 & 2014 & 57 & 1.179 & 1782 & 69 \\
\hline 16 & 1.361 & 2046 & 9 & 1.336 & 2154 & 53 & 1.361 & 2052 & 22 \\
\hline 17 & 1.014 & 2498 & 6 & 1.403 & 2539 & 61 & 1.403 & 2539 & 35 \\
\hline 18 & 1.41 & 2607 & 3547 & 1.41 & 2607 & 81 & 1.416 & 2564 & 249 \\
\hline 19 & 1.787 & 3111 & 12 & 1.762 & 3300 & 73 & 1.781 & 3140 & 75 \\
\hline 20 & 2.433 & 4060 & 1634 & 2.428 & 4117 & 291 & 2.439 & 4023 & 521 \\
\hline 21 & 3.175 & 5617 & 1029 & 3.164 & 5667 & 327 & 3.174 & 5630 & 1263 \\
\hline
\end{tabular}




\section{Appendix B}

\section{Results for Large Scale Problem}

\section{B.1 Instance 1}

Table B.1: Result obtained from the solver for large scale problem: Instance 1

\begin{tabular}{|c|c|c|c|c|c|c|c|c|c|}
\hline & \multicolumn{3}{|c|}{ Weighted Sum } & \multicolumn{3}{c|}{ Hierarchical } & \multicolumn{3}{c|}{ Trade-off } \\
\hline \hline Problem & $\begin{array}{c}\text { Delay } \\
(\mathrm{s})\end{array}$ & $\begin{array}{c}\text { Traffic } \\
(\mathrm{Mbps})\end{array}$ & $\begin{array}{c}\text { Solution Time } \\
(\mathrm{s})\end{array}$ & $\begin{array}{c}\text { Delay } \\
(\mathrm{s})\end{array}$ & $\begin{array}{c}\text { Traffic } \\
(\mathrm{Mbps})\end{array}$ & $\begin{array}{c}\text { Solution Time } \\
(\mathrm{s})\end{array}$ & $\begin{array}{c}\text { Delay } \\
(\mathrm{s})\end{array}$ & $\begin{array}{c}\text { Traffic } \\
(\text { Mbps })\end{array}$ & $\begin{array}{c}\text { Solution Time } \\
(\mathrm{s})\end{array}$ \\
\hline \hline 22 & 0.131 & 199 & 2 & 0.131 & 199 & 4 & 0.131 & 201 & 2 \\
\hline 23 & 0.223 & 104 & 11 & 0.223 & 104 & 13 & 0.223 & 104 & 4 \\
\hline 24 & 0.241 & 316 & 3 & 0.241 & 316 & 13 & 0.241 & 316 & 4 \\
\hline 25 & 0.345 & 310 & 140 & 0.338 & 336 & 18 & 0.345 & 310 & 71 \\
\hline 26 & 0.501 & 694 & 346 & 0.502 & 694 & 314 & 0.501 & 694 & 369 \\
\hline 27 & 0.495 & 680 & 456 & 0.494 & 711 & 3215 & 0.495 & 690 & 851 \\
\hline 28 & 0.548 & 648 & 404 & 0.529 & 783 & 1963 & 0.535 & 719 & 755 \\
\hline 29 & 0.69 & 764 & 553 & 0.684 & 877 & 2501 & 0.69 & 771 & 2369 \\
\hline 30 & 0.675 & 871 & 5258 & 0.675 & 880 & 4758 & 0.674 & 880 & 2781 \\
\hline 31 & 0.726 & 1135 & 14804 & 0.714 & 1249 & 1144 & 0.726 & 1135 & 1514 \\
\hline 32 & 0.833 & 893 & 30324 & 0.841 & 881 & 7023 & 0.845 & 853 & 6973 \\
\hline
\end{tabular}




\section{B.2 Instance 2}

Table B.2: Result obtained from the solver for large scale problem: Instance 2

\begin{tabular}{|c|c|c|c|c|c|c|c|c|c|}
\hline & \multicolumn{3}{|c|}{ Weighted Sum } & \multicolumn{3}{c|}{ Hierarchical } & \multicolumn{3}{c|}{ Trade-off } \\
\hline \hline Problem & $\begin{array}{c}\text { Delay } \\
(\mathrm{s})\end{array}$ & $\begin{array}{c}\text { Traffic } \\
(\mathrm{Mbps})\end{array}$ & $\begin{array}{c}\text { Solution Time } \\
(\mathrm{s})\end{array}$ & $\begin{array}{c}\text { Delay } \\
(\mathrm{s})\end{array}$ & $\begin{array}{c}\text { Traffic } \\
(\text { Mbps })\end{array}$ & $\begin{array}{c}\text { Solution Time } \\
(\mathrm{s})\end{array}$ & $\begin{array}{c}\text { Delay } \\
(\mathrm{s})\end{array}$ & $\begin{array}{c}\text { Traffic } \\
(\text { Mbps })\end{array}$ & $\begin{array}{c}\text { Solution Time } \\
(\mathrm{s})\end{array}$ \\
\hline \hline 22 & 0.09 & 149 & 2 & 0.09 & 149 & 4 & 0.09 & 149 & 2 \\
\hline 23 & 0.209 & 151 & 3 & 0.204 & 164 & 7 & 0.209 & 151 & 2 \\
\hline 24 & 0.243 & 160 & 726 & 0.237 & 197 & 412 & 0.242 & 170 & 10754 \\
\hline 25 & 0.294 & 479 & 7314 & 0.294 & 479 & 286 & 0.294 & 479 & 13442 \\
\hline 26 & 0.404 & 594 & 63 & 0.392 & 650 & 25 & 0.398 & 618 & 62 \\
\hline 27 & 0.54 & 545 & 3886 & 0.533 & 622 & 6190 & 0.533 & 622 & 1051 \\
\hline 28 & 0.605 & 834 & 2172 & 0.6 & 883 & 648 & 0.605 & 834 & 1111 \\
\hline 29 & 0.761 & 901 & 5401 & 0.755 & 943 & 2204 & 0.761 & 908 & 11549 \\
\hline 30 & 0.71 & 1052 & 1216 & 0.71 & 1052 & 205 & 0.71 & 1052 & 679 \\
\hline 31 & 0.841 & 1092 & 694 & 0.834 & 1178 & 7555 & 0.841 & 1092 & 6368 \\
\hline 32 & 0.993 & 1350 & 8233 & 0.943 & 1425 & 11234 & 0.942 & 1425 & 23316 \\
\hline
\end{tabular}




\section{B.3 Instance 3}

Table B.3: Result obtained from the solver for large scale problem: Instance 3

\begin{tabular}{|c|c|c|c|c|c|c|c|c|c|}
\hline & \multicolumn{3}{|c|}{ Weighted Sum } & \multicolumn{3}{c|}{ Hierarchical } & \multicolumn{3}{c|}{ Trade-off } \\
\hline \hline Problem & $\begin{array}{c}\text { Delay } \\
(\mathrm{s})\end{array}$ & $\begin{array}{c}\text { Traffic } \\
(\text { Mbps })\end{array}$ & $\begin{array}{c}\text { Solution Time } \\
(\mathrm{s})\end{array}$ & $\begin{array}{c}\text { Delay } \\
(\mathrm{s})\end{array}$ & $\begin{array}{c}\text { Traffic } \\
(\text { Mbps })\end{array}$ & $\begin{array}{c}\text { Solution Time } \\
(\mathrm{s})\end{array}$ & $\begin{array}{c}\text { Delay } \\
(\mathrm{s})\end{array}$ & $\begin{array}{c}\text { Traffic } \\
(\text { Mbps })\end{array}$ & $\begin{array}{c}\text { Solution Time } \\
(\mathrm{s})\end{array}$ \\
\hline \hline 22 & 0.16 & 116 & 2 & 0.16 & 116 & 4 & 0.16 & 116 & 2 \\
\hline 23 & 0.138 & 208 & 3 & 0.138 & 217 & 11 & 0.137 & 223 & 3 \\
\hline 24 & 0.261 & 206 & 301 & 0.262 & 206 & 59 & 0.261 & 206 & 34 \\
\hline 25 & 0.372 & 467 & 131 & 0.372 & 467 & 227 & 0.377 & 422 & 523 \\
\hline 26 & 0.432 & 687 & 4450 & 0.432 & 687 & 379 & 0.432 & 687 & 3255 \\
\hline 27 & 0.467 & 540 & 183 & 0.461 & 577 & 51 & 0.467 & 540 & 150 \\
\hline 28 & 0.592 & 648 & 272 & 0.585 & 718 & 796 & 0.592 & 648 & 351 \\
\hline 29 & 0.692 & 880 & 259 & 0.692 & 902 & 4250 & 0.692 & 880 & 504 \\
\hline 30 & 0.637 & 738 & 93 & 0.624 & 923 & 353 & 0.636 & 785 & 275 \\
\hline 31 & 0.782 & 552 & 1378 & 0.763 & 695 & 2297 & 0.776 & 587 & 9774 \\
\hline 32 & 0.84 & 1302 & 2463 & 0.811 & 1405 & 1340 & 0.833 & 1326 & 1492 \\
\hline
\end{tabular}




\section{B.4 Instance 4}

Table B.4: Result obtained from the solver for large scale problem: Instance 4

\begin{tabular}{|c|c|c|c|c|c|c|c|c|c|}
\hline & \multicolumn{3}{|c|}{ Weighted Sum } & \multicolumn{3}{c|}{ Hierarchical } & \multicolumn{3}{c|}{ Trade-off } \\
\hline \hline Problem & $\begin{array}{c}\text { Delay } \\
(\mathrm{s})\end{array}$ & $\begin{array}{c}\text { Traffic } \\
(\mathrm{Mbps})\end{array}$ & $\begin{array}{c}\text { Solution Time } \\
(\mathrm{s})\end{array}$ & $\begin{array}{c}\text { Delay } \\
(\mathrm{s})\end{array}$ & $\begin{array}{c}\text { Traffic } \\
(\text { Mbps })\end{array}$ & $\begin{array}{c}\text { Solution Time } \\
(\mathrm{s})\end{array}$ & $\begin{array}{c}\text { Delay } \\
(\mathrm{s})\end{array}$ & $\begin{array}{c}\text { Traffic } \\
(\text { Mbps })\end{array}$ & $\begin{array}{c}\text { Solution Time } \\
(\mathrm{s})\end{array}$ \\
\hline \hline 22 & 0.094 & 45 & 2 & 0.094 & 45 & 6 & 0.094 & 48 & 2 \\
\hline 23 & 0.22 & 165 & 57 & 0.22 & 170 & 19 & 0.22 & 185 & 13 \\
\hline 24 & 0.298 & 222 & 2 & 0.298 & 222 & 12 & 0.298 & 257 & 6 \\
\hline 25 & 0.355 & 166 & 16494 & 0.354 & 201 & 2694 & 0.355 & 166 & 27744 \\
\hline 26 & 0.458 & 455 & 59 & 0.452 & 490 & 560 & 0.458 & 455 & 57 \\
\hline 27 & 0.467 & 540 & 222 & 0.486 & 596 & 559 & 0.486 & 596 & 1152 \\
\hline 28 & 0.61 & 721 & 5592 & 0.603 & 755 & 3602 & 0.61 & 723 & 1565 \\
\hline 29 & 0.622 & 816 & 12234 & 0.616 & 908 & 8575 & 0.622 & 820 & 11233 \\
\hline 30 & 0.642 & 928 & 1434 & 0.63 & 1031 & 6029 & 0.636 & 978 & 2723 \\
\hline 31 & 0.784 & 998 & 7373 & 0.764 & 1111 & 2440 & 0.79 & 987 & 6664 \\
\hline 32 & 0.837 & 1236 & 7195 & 0.831 & 1305 & 16439 & 0.837 & 1236 & 1698 \\
\hline
\end{tabular}




\section{Appendix $\mathrm{C}$}

\section{Results for Single and Multiobjective}

\section{C.1 Instance 1}

Table C.1: Result for single objective and multiobjective: Instance 1

\begin{tabular}{|c|c|c|c|c|c|c|c|c|}
\hline & \multicolumn{2}{|c|}{ Single Objective } & \multicolumn{2}{|c|}{ Weighted Sum } & \multicolumn{2}{|c|}{ Hierarchical } & \multicolumn{2}{|c|}{ Trade-off } \\
\hline \hline Problem & $\begin{array}{c}\text { Delay } \\
(\mathbf{s})\end{array}$ & $\begin{array}{c}\text { Traffic } \\
(\text { Mbps })\end{array}$ & $\begin{array}{c}\text { Delay Gap } \\
(\%)\end{array}$ & $\begin{array}{c}\text { Trattic Gap } \\
(\%)\end{array}$ & $\begin{array}{c}\text { Delay Gap } \\
(\%)\end{array}$ & $\begin{array}{c}\text { Traftic Gap } \\
(\%)\end{array}$ & $\begin{array}{c}\text { Delay Gap } \\
(\%)\end{array}$ & $\begin{array}{c}\text { Traftic Gap } \\
\text { (\%) }\end{array}$ \\
\hline \hline 1 & 0.098 & 67 & 6.3 & 0 & 0 & 65 & 6.2 & 16 \\
\hline 2 & 0.177 & 111 & 0.29 & 4.9 & 0.34 & 4.9 & 0.29 & 4.9 \\
\hline 3 & 0.27 & 443 & 2.5 & 1.1 & 0.0072 & 6.9 & 2.5 & 1.1 \\
\hline 4 & 0.347 & 542 & 1.9 & 2.2 & 0.097 & 9.7 & 1.9 & 2.2 \\
\hline 5 & 0.386 & 564 & 0.08 & 12 & 0.08 & 12 & 0.08 & 12 \\
\hline 6 & 0.576 & 806 & 1.1 & 1.2 & 0.00034 & 6.3 & 1.1 & 1.2 \\
\hline 7 & 0.65 & 1073 & 0.14 & 5.2 & 0.14 & 5.2 & 0.14 & 5.2 \\
\hline 8 & 0.62 & 953 & 7.1 & 0 & 0.07 & 12 & 1 & 6 \\
\hline 9 & 0.665 & 1191 & 0.96 & 4.7 & 0.049 & 11 & 0.92 & 5.5 \\
\hline 10 & 0.785 & 1172 & 0.81 & 4.5 & 0.082 & 10 & 0.81 & 4.5 \\
\hline 11 & 0.983 & 1523 & 1.4 & 3.5 & 0.097 & 7.9 & 0.74 & 5.6 \\
\hline 12 & 1.106 & 1845 & 1.1 & 0.44 & 0.0091 & 3.9 & 1.2 & 0.29 \\
\hline 13 & 1.054 & 1765 & 0.65 & 3.4 & 0.025 & 8.5 & 0.65 & 3.4 \\
\hline 14 & 1.1 & 1826 & 1.1 & 2.9 & 0.61 & 4.5 & 1.1 & 2.9 \\
\hline 15 & 1.298 & 2038 & 0.51 & 3 & 0.5 & 3.1 & 0.46 & 3.1 \\
\hline 16 & 1.253 & 2165 & 1 & 4.8 & 0.13 & 11 & 0.99 & 6.1 \\
\hline 17 & 1.472 & 2482 & 0.47 & 5.9 & 0.49 & 5.9 & 0.47 & 6 \\
\hline 18 & 1.388 & 2518 & 0.96 & 1.7 & 0.08 & 5 & 0.96 & 1.7 \\
\hline 19 & 1.531 & 2674 & 0.42 & 2.2 & 0.48 & 2.2 & 0.42 & 2.5 \\
\hline 20 & 2.407 & 4383 & 0.54 & 2.7 & 0.35 & 3.4 & 0.81 & 2.2 \\
\hline 21 & 3.11 & 5263 & 0.65 & 1.8 & 0.47 & 2.3 & 0.82 & 1.6 \\
\hline 22 & 0.131 & 199 & 0.098 & 0 & 0.098 & 0 & 0.057 & 1.3 \\
\hline 23 & 0.223 & 93 & 0.051 & 12 & 0.066 & 12 & 0.051 & 12 \\
\hline 24 & 0.241 & 316 & 0.038 & 0 & 0.038 & 0 & 0.038 & 0 \\
\hline 25 & 0.338 & 300 & 2 & 3.4 & 0.0091 & 12 & 2 & 3.4 \\
\hline 26 & 0.501 & 672 & 0.068 & 3.3 & 0.088 & 3.3 & 0.068 & 3.3 \\
\hline 27 & 0.494 & 647 & 0.2 & 5.2 & 0.089 & 10 & 0.2 & 6.7 \\
\hline 28 & 0.529 & 622 & 3.6 & 4.1 & 0.099 & 26 & 1.3 & 16 \\
\hline 29 & 0.684 & 705 & 0.84 & 8.4 & 0.083 & 25 & 0.83 & 9.4 \\
\hline 30 & 0.673 & 807 & 0.26 & 8 & 0.19 & 9.1 & 0.17 & 9.1 \\
\hline 31 & 0.713 & 1066 & 1.8 & 6.4 & 0.096 & 17 & 1.8 & 6.4 \\
\hline 32 & 0.833 & 796 & 0.07 & 12 & 1 & 11 & 1.5 & 7.1 \\
\hline
\end{tabular}




\section{C.2 Instance 2}

Table C.2: Result for single objective and multiobjective: Instance 2

\begin{tabular}{|c|c|c|c|c|c|c|c|c|}
\hline & \multicolumn{2}{|c|}{ Single Objective } & \multicolumn{2}{|c|}{ Weighted Sum } & \multicolumn{2}{|c|}{ Hierarchical } & \multicolumn{2}{|c|}{ Trade-off } \\
\hline Problem & $\begin{array}{l}\text { Delay } \\
\text { (s) }\end{array}$ & $\begin{array}{l}\text { Traffic } \\
\text { (Mbps) }\end{array}$ & $\begin{array}{c}\text { Delay Gap } \\
(\%)\end{array}$ & $\begin{array}{c}\text { Traffic Gap } \\
(\%)\end{array}$ & $\begin{array}{c}\text { Delay Gap } \\
(\%)\end{array}$ & $\begin{array}{c}\text { Traffic Gap } \\
(\%)\end{array}$ & $\begin{array}{c}\text { Delay Gap } \\
(\%)\end{array}$ & $\begin{array}{c}\text { Traffic Gap } \\
(\%)\end{array}$ \\
\hline 1 & 0.068 & 11 & 0 & 0 & 0 & 0 & 0 & 0 \\
\hline 2 & 0.224 & 250 & 0.1 & 11 & 0.1 & 11 & 0.1 & 11 \\
\hline 3 & 0.269 & 412 & 0.038 & 2 & 0.038 & 2 & 0.038 & 2 \\
\hline 4 & 0.4 & 406 & 0.048 & 7.1 & 0.048 & 7.1 & 0.048 & 7.1 \\
\hline 5 & 0.444 & 447 & 0.046 & 10 & 0.046 & 10 & 1.4 & 5.1 \\
\hline 6 & 0.543 & 894 & 1.2 & 1.8 & 0.022 & 11 & 1.2 & 1.8 \\
\hline 7 & 0.628 & 888 & 0.98 & 5.6 & 0.088 & 8.3 & 1.9 & 4.2 \\
\hline 8 & 0.578 & 932 & 1.1 & 0 & 0.04 & 14 & 1.1 & 5.8 \\
\hline 9 & 0.695 & 1225 & 0.97 & 1.8 & 0.043 & 4.4 & 0.95 & 1.9 \\
\hline 10 & 0.811 & 1223 & 0.77 & 6.5 & 0 & 12 & 0.77 & 6.5 \\
\hline 11 & 0.816 & 1253 & 0.79 & 4.7 & 0 & 15 & 0.79 & 4.4 \\
\hline 12 & 0.909 & 1414 & 0.75 & 3.2 & 0.049 & 6.9 & 0.75 & 3.2 \\
\hline 13 & 1.153 & 1921 & 0.61 & 3.9 & 0.054 & 6.8 & 0.61 & 3.9 \\
\hline 14 & 1.158 & 2000 & 1.6 & 2.5 & 0.02 & 11 & 1.6 & 2.5 \\
\hline 15 & 1.186 & 2132 & 1 & 3.8 & 0.49 & 5.7 & 1.6 & 2.7 \\
\hline 16 & 1.308 & 1968 & 0.5 & 3.9 & 0.046 & 6 & 0.5 & 3.9 \\
\hline 17 & 1.258 & 1655 & 1.5 & 3.5 & 0.48 & 7.4 & 1.5 & 4.2 \\
\hline 18 & 1.616 & 2972 & 0.79 & 1.2 & 0.49 & 2.1 & 0.79 & 1.2 \\
\hline 19 & 1.519 & 3232 & 2.1 & 1.1 & 0.45 & 3.4 & 1.6 & 1.9 \\
\hline 20 & 2.365 & 3880 & 0.82 & 1.5 & 0.41 & 3.4 & 0.82 & 1.6 \\
\hline 21 & 3.305 & 5599 & 0.4 & 1.5 & 0.2 & 2.3 & 0.59 & 1.2 \\
\hline 22 & 0.09 & 149 & 0 & 0 & 0.0068 & 0 & 0 & 0 \\
\hline 23 & 0.204 & 151 & 2.8 & 0 & 0.0014 & 8.5 & 2.8 & 0 \\
\hline 24 & 0.237 & 160 & 2.5 & 0 & 0 & 0 & 2 & 0 \\
\hline 25 & 0.294 & 427 & 0.13 & 12 & 0.13 & 12 & 0.13 & 12 \\
\hline 26 & 0.391 & 580 & 3.3 & 2.4 & 0.096 & 12 & 1.7 & 6.6 \\
\hline 27 & 0.533 & 481 & 1.5 & 13 & 0.13 & 29 & 0.11 & 29 \\
\hline 28 & 0.599 & 788 & 1 & 5.8 & 0.15 & 12 & 1 & 5.8 \\
\hline 29 & 0.754 & 866 & 0.96 & 4 & 0.077 & 8.9 & 0.87 & 4.9 \\
\hline 30 & 0.709 & 957 & 0.12 & 9.9 & 0.14 & 9.9 & 0.12 & 9.9 \\
\hline 31 & 0.834 & 985 & 0.86 & 11 & 0.039 & 20 & 0.86 & 11 \\
\hline 32 & 0.941 & 1350 & 5.5 & 0.000027 & 0.16 & 5.6 & 0.13 & 5.6 \\
\hline
\end{tabular}




\section{C.3 Instance 3}

Table C.3: Result for single objective and multiobjective: Instance 3

\begin{tabular}{|c|c|c|c|c|c|c|c|c|}
\hline & \multicolumn{2}{|c|}{ Single Objective } & \multicolumn{2}{|c|}{ Weighted Sum } & \multicolumn{2}{|c|}{ Hierarchical } & \multicolumn{2}{|c|}{ Trade-off } \\
\hline Problem & $\begin{array}{l}\text { Delay } \\
\text { (s) }\end{array}$ & $\begin{array}{l}\text { Traffic } \\
\text { (Mbps) }\end{array}$ & $\begin{array}{c}\text { Delay Gap } \\
(\%)\end{array}$ & $\begin{array}{c}\text { Traffic Gap } \\
(\%)\end{array}$ & $\begin{array}{c}\text { Delay Gap } \\
(\%)\end{array}$ & $\begin{array}{c}\text { Traffic Gap } \\
(\%)\end{array}$ & $\begin{array}{c}\text { Delay Gap } \\
(\%)\end{array}$ & $\begin{array}{c}\text { Traffic Gap } \\
(\%)\end{array}$ \\
\hline 1 & 0.167 & 255 & 0.075 & 0.0000079 & 0.075 & 0 & 0.039 & 0.0000079 \\
\hline 2 & 0.177 & 145 & 0.39 & 7.1 & 0.43 & 7.1 & 0.39 & 7.1 \\
\hline 3 & 0.287 & 341 & 0.074 & 3.2 & 0.074 & 3.2 & 0.074 & 3.2 \\
\hline 4 & 0.255 & 226 & 2.4 & 6.8 & 0.026 & 18 & 2.4 & 6.8 \\
\hline 5 & 0.433 & 630 & 1.6 & 2.2 & 0.057 & 11 & 1.6 & 2.2 \\
\hline 6 & 0.554 & 766 & 0.077 & 4.3 & 0.095 & 4.3 & 0.062 & 4.5 \\
\hline 7 & 0.518 & 586 & 2.4 & 4.6 & 0.018 & 15 & 1.2 & 10 \\
\hline 8 & 0.736 & 1267 & 0.067 & 3 & 0.089 & 3 & 0.067 & 3 \\
\hline 9 & 0.726 & 1154 & 0.9 & 1.8 & 0.028 & 4.9 & 0.9 & 1.8 \\
\hline 10 & 0.92 & 1599 & 0.68 & 2.1 & 0.085 & 5 & 0.68 & 2.1 \\
\hline 11 & 1.002 & 1637 & 1.3 & 1.4 & 0.038 & 6.2 & 1.3 & 1.4 \\
\hline 12 & 0.952 & 1393 & 0.77 & 4.1 & 0.028 & 10 & 0.77 & 2.9 \\
\hline 13 & 0.987 & 1515 & 0.79 & 6.1 & 3.9 & 0 & 0.66 & 7.8 \\
\hline 14 & 1.268 & 1957 & 0.053 & 3.8 & 0.073 & 3.8 & 0.51 & 2.9 \\
\hline 15 & 1.169 & 1824 & 1.1 & 3.6 & 0.5 & 7.8 & 1.1 & 3.6 \\
\hline 16 & 1.379 & 2580 & 0.44 & 5.2 & 0.49 & 5.2 & 0.44 & 5.2 \\
\hline 17 & 0.899 & 2076 & 0.69 & 2.5 & 42 & 5.4 & 43 & 3.8 \\
\hline 18 & 0.993 & 2592 & 0.32 & 1.2 & 0.19 & 2.6 & 0.12 & 3.5 \\
\hline 19 & 1.573 & 2901 & 1.2 & 1.9 & 0.49 & 4.7 & 1.2 & 1.9 \\
\hline 20 & 2.342 & 3501 & 0.54 & 1.6 & 0.34 & 2 & 0.54 & 1.6 \\
\hline 21 & 3.237 & 5940 & 0.6 & 1.4 & 0.46 & 1.8 & 0.4 & 1.8 \\
\hline 22 & 0.16 & 116 & 0 & 0 & 0 & 0 & 0 & 0 \\
\hline 23 & 0.137 & 208 & 0.52 & 0 & 0.5 & 4.5 & 0.33 & 7.4 \\
\hline 24 & 0.261 & 198 & 0.07 & 3.7 & 0.097 & 3.7 & 0.07 & 3.7 \\
\hline 25 & 0.372 & 384 & 0.1 & 22 & 0.1 & 22 & 1.6 & 9.8 \\
\hline 26 & 0.431 & 671 & 0.11 & 2.2 & 0.14 & 2.2 & 0.11 & 2.2 \\
\hline 27 & 0.461 & 538 & 1.4 & 0.44 & 0.0087 & 7.4 & 1.4 & 0.44 \\
\hline 28 & 0.584 & 617 & 1.2 & 5.2 & 0.086 & 16 & 1.2 & 5.2 \\
\hline 29 & 0.691 & 849 & 0.15 & 3.7 & 0.096 & 6.3 & 0.15 & 3.7 \\
\hline 30 & 0.624 & 733 & 2.1 & 0.74 & 0.085 & 26 & 2 & 7.1 \\
\hline 31 & 0.761 & 512 & 2.6 & 7.9 & 0.14 & 36 & 1.9 & 15 \\
\hline 32 & 0.807 & 1272 & 4.1 & 2.4 & 0.47 & 10 & 3.2 & 4.3 \\
\hline
\end{tabular}




\section{C.4 Instance 4}

Table C.4: Result for single objective and multiobjective: Instance 4

\begin{tabular}{|c|c|c|c|c|c|c|c|c|}
\hline & \multicolumn{2}{|c|}{ Single Objective } & \multicolumn{2}{|c|}{ Weighted Sum } & \multicolumn{2}{|c|}{ Hierarchical } & \multicolumn{2}{|c|}{ Trade-off } \\
\hline Problem & $\begin{array}{l}\text { Delay } \\
\text { (s) }\end{array}$ & $\begin{array}{l}\text { Traffic } \\
\text { (Mbps) }\end{array}$ & $\begin{array}{c}\text { Delay Gap } \\
(\%)\end{array}$ & $\begin{array}{c}\text { Traffic Gap } \\
(\%)\end{array}$ & $\begin{array}{c}\text { Delay Gap } \\
(\%)\end{array}$ & $\begin{array}{c}\text { Traffic Gap } \\
(\%)\end{array}$ & $\begin{array}{c}\text { Delay Gap } \\
(\%)\end{array}$ & $\begin{array}{c}\text { Traffic Gap } \\
(\%)\end{array}$ \\
\hline 1 & 0.111 & 46 & 0.39 & 23 & 0.13 & 40 & 0.39 & 23 \\
\hline 2 & 0.185 & 146 & 3.4 & 4.5 & 0 & 3.6 & 3.4 & 4.5 \\
\hline 3 & 0.232 & 365 & 0.16 & 5.2 & 0.1 & 15 & 0.16 & 5.2 \\
\hline 4 & 0.358 & 304 & 0.16 & 10 & 0.18 & 10 & 0.16 & 10 \\
\hline 5 & 0.393 & 638 & 3.1 & 1.5 & 0 & 13 & 3.1 & 2 \\
\hline 6 & 0.619 & 1131 & 0.053 & 1.9 & 0.056 & 1.9 & 0.053 & 1.9 \\
\hline 7 & 0.467 & 855 & 1.4 & 6.8 & 5.5 & 0 & 1.4 & 6.8 \\
\hline 8 & 0.685 & 1126 & 0.97 & 6.6 & 0.097 & 10 & 0.97 & 6.6 \\
\hline 9 & 0.691 & 1217 & 0.94 & 4.1 & 0.031 & 8.6 & 1.8 & 2.3 \\
\hline 10 & 0.751 & 1294 & 0.032 & 2.9 & 0.036 & 2.9 & 0.016 & 3.3 \\
\hline 11 & 0.922 & 1523 & 0.75 & 4 & 0.058 & 5.8 & 0.7 & 4.8 \\
\hline 12 & 0.984 & 1824 & 2 & 4.1 & 0.088 & 10 & 1.3 & 6.5 \\
\hline 13 & 1.04 & 1646 & 1.2 & 2 & 3.7 & 0 & 1.2 & 2.6 \\
\hline 14 & 1.106 & 1830 & 1.2 & 2.5 & 0.022 & 7.5 & 1.2 & 2.9 \\
\hline 15 & 1.142 & 1750 & 1.1 & 7.6 & 0.013 & 15 & 3.3 & 1.8 \\
\hline 16 & 1.336 & 2022 & 1.9 & 1.2 & 0.017 & 6.5 & 1.9 & 1.5 \\
\hline 17 & 1.004 & 2485 & 1 & 0.53 & 40 & 2.2 & 40 & 2.2 \\
\hline 18 & 1.409 & 2505 & 0.06 & 4.1 & 0.066 & 4.1 & 0.5 & 2.4 \\
\hline 19 & 1.762 & 3041 & 1.4 & 2.3 & 0.019 & 8.5 & 1.1 & 3.3 \\
\hline 20 & 2.419 & 3912 & 0.58 & 3.8 & 0.36 & 5.3 & 0.82 & 2.8 \\
\hline 21 & 3.149 & 5567 & 0.82 & 0.91 & 0.47 & 1.8 & 0.79 & 1.1 \\
\hline 22 & 0.094 & 45 & 0.025 & 0 & 0.05 & 0 & 0.016 & 8.1 \\
\hline 23 & 0.22 & 165 & 0.22 & 0 & 0.18 & 3.1 & 0.091 & 12 \\
\hline 24 & 0.298 & 222 & 0.057 & 0 & 0.079 & 0 & 0 & 16 \\
\hline 25 & 0.354 & 155 & 0.41 & 7.1 & 0.17 & 30 & 0.41 & 7.1 \\
\hline 26 & 0.452 & 406 & 1.5 & 12 & 0.14 & 21 & 1.5 & 12 \\
\hline 27 & 0.485 & 545 & 3.8 & 8.2 & 0.097 & 9.4 & 0.097 & 9.4 \\
\hline 28 & 0.603 & 690 & 1.1 & 4.6 & 0.017 & 9.5 & 1.1 & 4.9 \\
\hline 29 & 0.615 & 719 & 1.1 & 14 & 0.14 & 26 & 1.1 & 14 \\
\hline 30 & 0.629 & 847 & 2.1 & 9.5 & 0.2 & 22 & 1.1 & 15 \\
\hline 31 & 0.763 & 933 & 2.7 & 6.9 & 0.13 & 19 & 3.5 & 5.7 \\
\hline 32 & 0.829 & 1160 & 0.88 & 6.6 & 0.15 & 13 & 0.88 & 6.6 \\
\hline
\end{tabular}

